\title{
Ligand-Induced Haptotropic Rearrangements in Bis(indenyl)zirconium Sandwich Complexes
}

\author{
Christopher A. Bradley, Emil Lobkovsky, Ivan Keresztes, and Paul J. Chirik* \\ pc92@cornell.edu \\ Department of Chemistry and Chemical Biology, Baker Laboratory \\ Cornell University, Ithaca, NY 14853
}

- $\quad$ Supporting Information- 


\section{Table of Contents}

EXSY NMR spectrum for $\mathbf{1}+\mathrm{THF} / \mathbf{1 - T H F} \quad$ S3

$\begin{array}{ll}\text { Van't Hoff plots for THF coordination to } \mathbf{1} \text { and } \mathbf{7} & \text { S4 }\end{array}$

Plots of EXSY crosspeak intensity versus mixing time S5

Selected chemical shifts of $\square^{6}, \square^{5}$ bis(indenyl)zirconium ligand complexes $\quad$ S6

$\begin{array}{ll}\text { Additional experimental procedures } & \text { S7 }\end{array}$

$\begin{array}{lr}\text { Crystallographic data for 2-THF } & \text { S18 }\end{array}$

$\begin{array}{lr}\text { Crystallographic data for } r a c-4-T H F & \text { S24 }\end{array}$

$\begin{array}{lr}\text { Crystallographic data for 1-DME } & \text { S30 }\end{array}$

$\begin{array}{ll}\text { Labeled view of the molecular structure of 1-THF } & \text { S43 }\end{array}$

$\begin{array}{lr}\text { Partial molecular orbital diagram of 1-THF } & \text { S44 }\end{array}$

$\begin{array}{ll}\text { References } & \text { S45 }\end{array}$ 

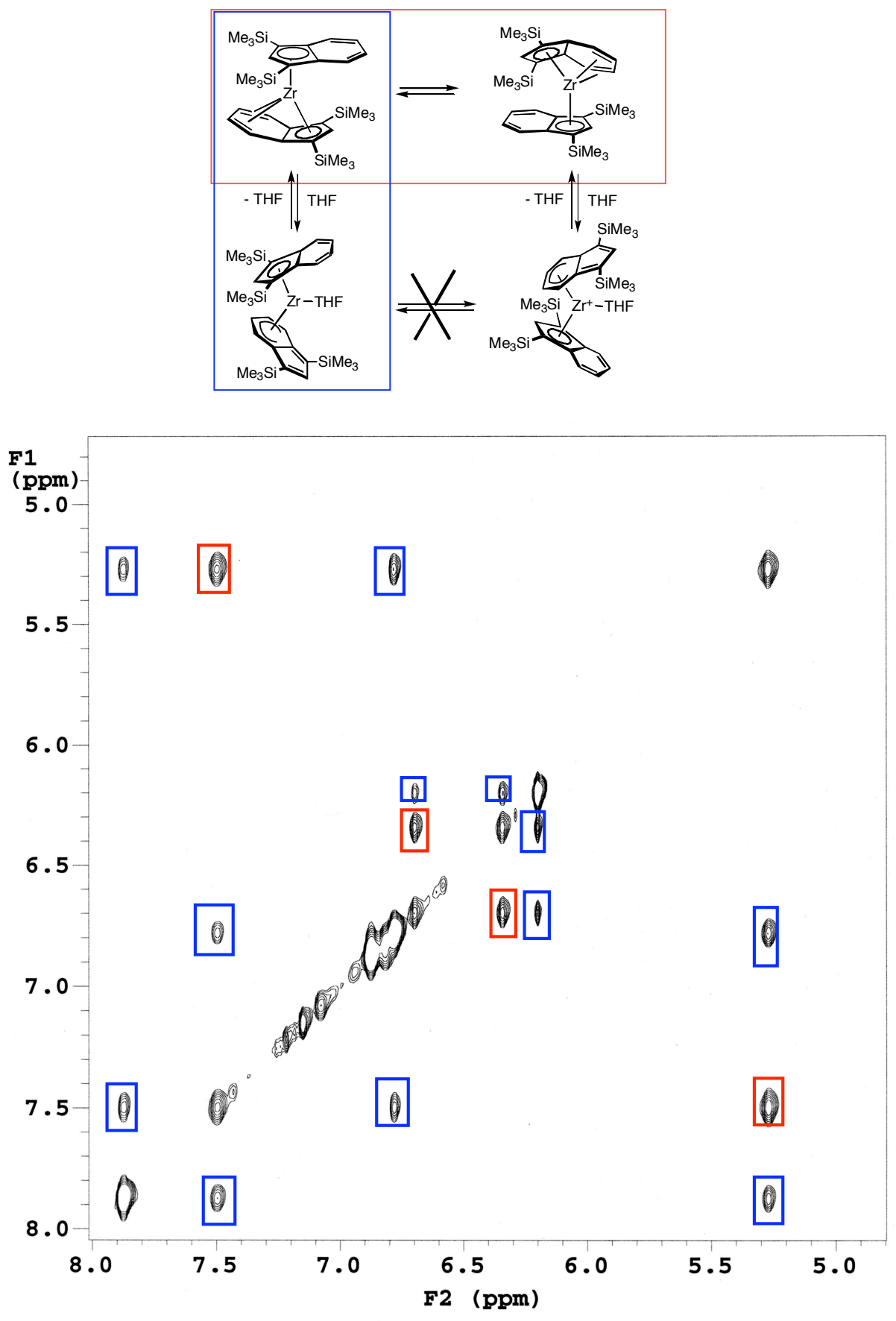

Figure S1. Cyclopentadienyl and benzo region of an EXSY NMR spectrum of $\mathbf{1}$ and 1-THF $\left(23{ }^{\circ} \mathrm{C}, 200\right.$ ms mixing time). Self exchange is denoted by red while reversible THF coordination is represented by blue boxes. 


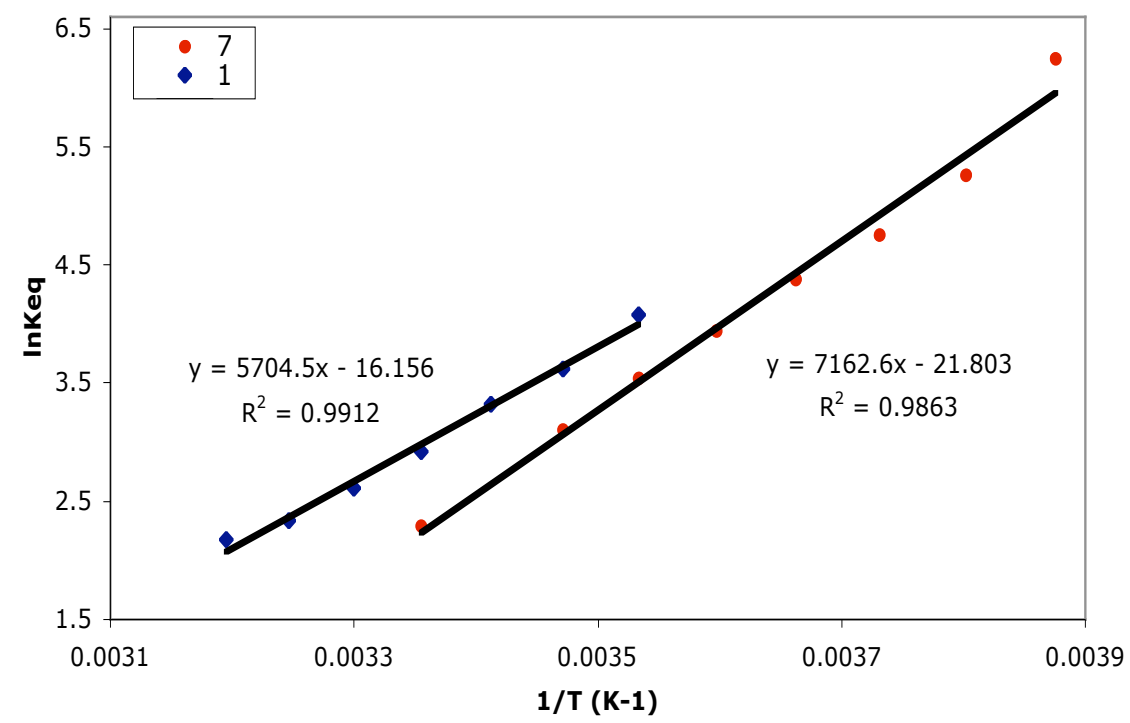

Figure S2. van't Hoff plots for THF coordination to $\mathbf{1}$ and $\mathbf{7}$. 
a)

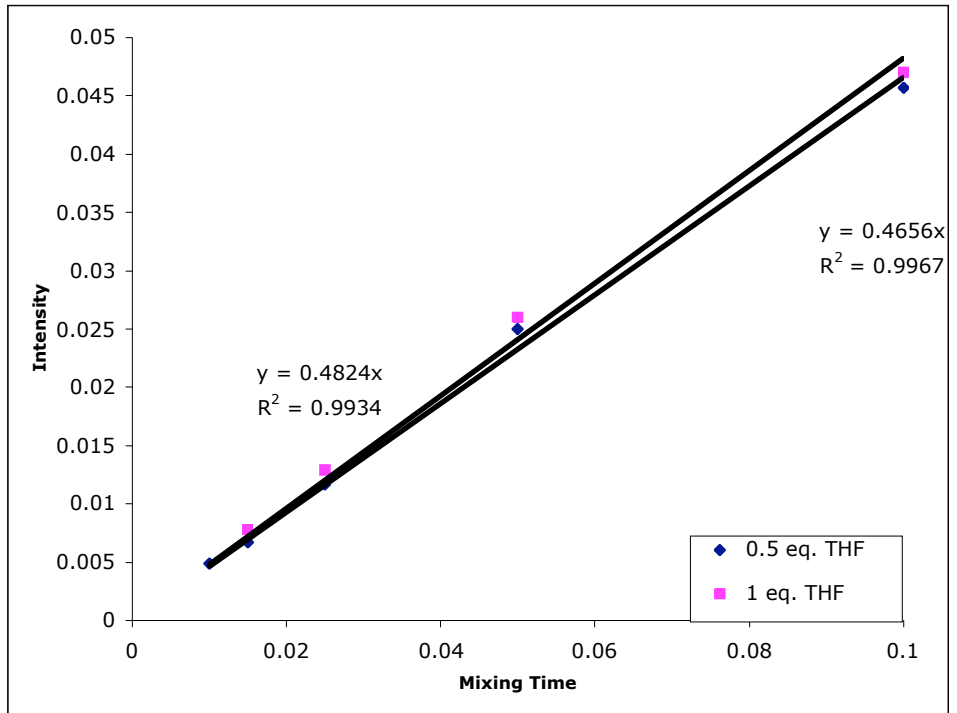

b)

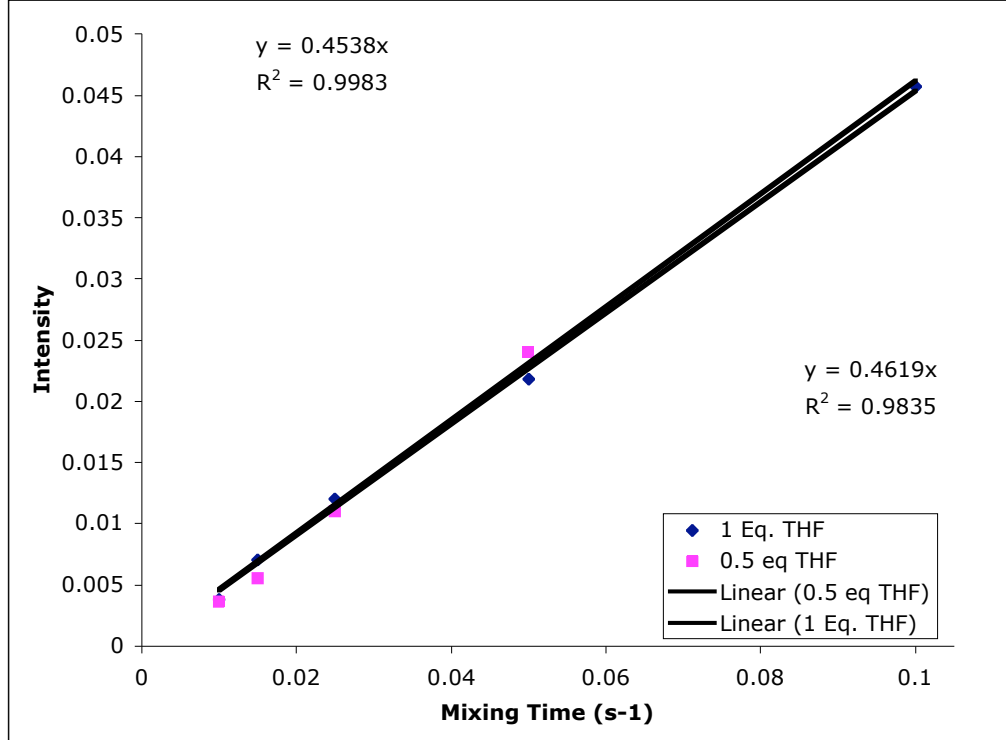

Figure S3. Plots of crosspeak volume versus mixing time for a) dissociation of THF from 1-THF and b) addition of THF to 1 at $23{ }^{\circ} \mathrm{C}$. 


\begin{tabular}{|c|c|c|c|c|c|c|}
\hline & \multicolumn{3}{|c|}{${ }^{1} \mathrm{H}$ shifts $(\mathrm{ppm})$} & \multicolumn{3}{|c|}{${ }^{13} \mathrm{C}$ Shifts (ppm) } \\
\hline & $\square^{5} \mathrm{Cp}$ & $\square^{6} \mathrm{Cp}$ & Benzo $_{p}$ & Benzo $_{d}$ & $\square^{6} \mathrm{Cp}$ & Benzo $_{p}$ \\
\hline 1-THF & 3.45 & 6.2 & 6.8 & 3.61 & 140.75 & 85.63 \\
\hline 2-THF & & & & & & \\
\hline 3-THF & 3.22 & 6.12 & 6.78 & 3.71 & $*$ & 85.01 \\
\hline 4-THF & $3.42(3.45)$ & $5.81(5.82)$ & $6.48(6.80)$ & $3.65(3.70)$ & $131-137$ & $84.99(86.03)$ \\
\hline 6-THF & $3.36(3.40)$ & $6.31(6.32)$ & $6.72(6.75)$ & $3.06(3.50)$ & $139-143$ & $85.74(86.20)$ \\
\hline 7-THF & 3.37 & $5.84(6.16)$ & $6.46-6.75$ & $3.63-3.73$ & $135-140$ & $83.96(85.49)$ \\
\hline $\begin{array}{l}\text { 1-OMe } \\
\text { 1-DME }\end{array}$ & 3.36 & 6.26 & 6.77 & 3.55 & - & - \\
\hline 1-DEE & 4.13 & 6.39 & 6.88 & 3.85 & 136.65 & 82.58 \\
\hline 1-rac-DMB & 4.16 & 6.79 & $6.33-6.44$ & 3.89 & $\begin{array}{r}135.97 \\
(140.39)\end{array}$ & $82.87(83.52)$ \\
\hline 1-DMM & 3.84 & 6.7 & 6.58 & 3.67 & 136.87 & 84.14 \\
\hline 1-DEM & 3.89 & 6.73 & 6.49 & 3.89 & - & - \\
\hline 2-DME & 4.12 & 7.11 & 6.31 & 3.65 & 143.93 & 83.33 \\
\hline 2-DMM & 3.84 & 6.93 & 6.63 & 3.31 & 143.58 & 85.51 \\
\hline 5-DME & 3.89 & 5.81 & 5.9 & 3.77 & - & - \\
\hline
\end{tabular}

Spectra recorded in either benzene- $d_{6}$ or toluene- $d_{8} *$ resonance not located; $-{ }^{13} \mathrm{C}$ data not collected. Benzo $=$ benzo

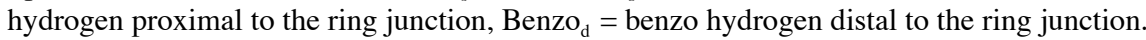

Table S1. Selected ${ }^{1} \mathrm{H}$ and ${ }^{13} \mathrm{C}$ NMR chemical shifts for $\square^{6}, \square^{5}$ bis(indenyl)zirconium ligand complexes. 


\section{Additional Experimental Procedures}

General Considerations. All air- and moisture-sensitive manipulations were carried out using standard vacuum line, Schlenk or cannula techniques or in an M. Braun inert atmosphere drybox containing an atmosphere of purified nitrogen. Solvents for air- and moisture-sensitive manipulations were initially dried and deoxygenated using literature procedures. ${ }^{1}$ Benzene- $d_{6}$ and toluene- $d_{8}$ for NMR spectroscopy were distilled from sodium metal under an atmosphere of argon and stored over $4 \AA$ molecular sieves or sodium metal. Dimethoxymethane, 1,2-dimethoxyethane, and 1,2-diethoxyethane were purchased from Acros Organics and dried over sodium benzophenone ketyl. Dimethyl ether was purchased from TCI America and dried over sodium benzophenone ketyl in a thick walled glass vessel prior to use. 1,2-Bis(dimethyl)phosphinoethane was purchased from Strem and used as received. 1-6, ${ }^{2}$ 1-THF, ${ }^{3}$ dimethoxyethylene ${ }^{4}$ and 1,2(dimethoxy)butane ${ }^{5}$ were prepared according to literature procedures. All calibrated gas bulb experiments were conducted using a $31.6 \mathrm{~mL}$ bulb unless noted otherwise.

${ }^{1} \mathrm{H}$ and ${ }^{13} \mathrm{C}$ NMR spectra were recorded on a Varian Inova 400 Spectrometer operating at $399.779 \mathrm{MHz}\left({ }^{1} \mathrm{H}\right)$ and $110.524 \mathrm{MHz}\left({ }^{13} \mathrm{C}\right)$. All chemical shifts are reported relative to $\mathrm{SiMe}_{4}$ using ${ }^{1} \mathrm{H}$ (residual) or ${ }^{13} \mathrm{C}$ NMR chemical shifts of the solvent as a secondary standard. EXSY and variable temperature experiments were recorded on a Varian 500 Inova spectrometer operating at $499.920 \mathrm{MHz}$ for ${ }^{1} \mathrm{H}$ and $125.704 \mathrm{MHz}$ for ${ }^{13} \mathrm{C} .{ }^{2} \mathrm{H}$ NMR spectra were recorded on a Varian Inova 500 spectrometer operating at 76.740 $\mathrm{MHz}$ and the spectra were referenced using an internal benzene- $d_{6}$ standard. ${ }^{31} \mathrm{P}$ NMR spectra were collected on a Varian Inova 400 Spectrometer and were externally referenced to $85 \% \mathrm{H}_{3} \mathrm{PO}_{4}$. 
Qualitative EXSY spectra were acquired with a sweep width of $5.4 \mathrm{kHz}$ and a mixing time of $200 \mathrm{~ms}$ in phase-sensitive mode. A total of 200 complex points were collected in the indirectly detected dimension with 16 scans and $2 \mathrm{k}$ points per increment. The resulting matrix was zero filled to $1 \mathrm{k}$ x $1 \mathrm{k}$ complex data points and Gaussian line broadening window functions were applied in both dimensions prior to Fourier transform. Magnetization transfer experiments were conducted with $0.035 \mathrm{M}$ of $\mathbf{1}$ containing 1, 5, 11, 17 and 25 equivalents of THF. The free and coordinated THF resonances were selectively excited using an IBURP2 shaped pulse with a pulse length of $44 \mathrm{~ms}$. Between 150-200 spectra were recorded with the inversion delay incremented from 0 to $2-3 \mathrm{~s}$ in 3.5 ms steps. Spectra were integrated using VNMR software (Version 1.1c, Varian Inc.) and processed with the CIFIT simulation program.

Gradient selected COSY spectra were acquired with a sweep width of $5.4 \mathrm{kHz}$. A total of 128 points were collected in the indirectly detected dimension with 4 scans and $2 \mathrm{k}$ points per increment. The resulting matrix was zero filled to $1 \mathrm{k} \mathrm{x} 1 \mathrm{k}$ complex data points and squared sinusoidal window functions were applied in both dimensions prior to Fourier transform. The quantitative 1-D EXSY rate studies were conducted with mixing times of 100, 50, 25, 5 and $1 \mathrm{~ms}$. Cross peak integreations were performed with Mest$\mathrm{ReC}^{6}$ and intensities were normalized and the corresponding rates calculated using literature procedures. ${ }^{7}$

Gradient selected HSQC spectra were acquired with sweep widths of $5.6 \mathrm{kHz}$ and $21.4 \mathrm{kHz}$ in ${ }^{1} \mathrm{H}$ and ${ }^{13} \mathrm{C}$ dimensions respectively. A total of 200 complex points were collected in the indirectly detected dimension with 12 scans and 2k points per increment. 
The resulting matrix was zero filled to $1 \mathrm{k} \mathrm{x} 1 \mathrm{k}$ complex data points and Gaussian line broadening window functions were applied in both dimensions prior to Fourier transform. Gradient selected HMBC spectra were acquired in absolute value mode with sweep widths of $5.6 \mathrm{kHz}$ and $30.2 \mathrm{kHz}$ in ${ }^{1} \mathrm{H}$ and ${ }^{13} \mathrm{C}$ dimensions respectively. A total of 500 complex points were collected in the indirectly detected dimension with 16 scans and $2 \mathrm{k}$ points per increment. The resulting matrix was zero filled to $1 \mathrm{k} x 1 \mathrm{k}$ complex data points and sinusoidal window functions were applied in both dimensions prior to Fourier transform.

NOESY spectra at $23{ }^{\circ} \mathrm{C}$ were acquired with a sweep width of $5.4 \mathrm{kHz}$ and a mixing time of $200 \mathrm{~ms}$ in phase-sensitive mode. A total of 200 complex points were collected in the indirectly detected dimension with 16 scans and $2 \mathrm{k}$ points per increment. The resulting matrix was zero filled to $1 \mathrm{k}$ x $1 \mathrm{k}$ complex data points and Gaussian line broadening window functions were applied in both dimensions prior to Fourier transform. All other 2D spectra were acquired at $23{ }^{\circ} \mathrm{C}$ unless otherwise noted.

Single crystals suitable for X-ray diffraction were coated with polyisobutylene oil in a drybox and were quickly transferred to the goniometer head of a Siemens SMART CCD Area detector system equipped with a molybdenum X-ray tube $(\square=0.71073 \AA$ ). Preliminary data revealed the crystal system. A hemisphere routine was used for data collection and determination of lattice constants. The space group was identified and the data were processed using the Bruker SAINT program and corrected for absorption using SADABS. The structures were solved using direct methods (SHELXS) completed by subsequent Fourier synthesis and refined by full-matrix least-squares procedures. 
Elemental analyses were performed at Robertson Microlit Laboratories, Inc., in Madison, NJ.

Preparation of $\left(\square^{6}-\mathrm{C}_{9} \mathrm{H}_{5}-1,3-\left(\mathrm{SiMe}_{2}{ }^{\mathrm{t}} \mathrm{Bu}\right)_{2}\right)\left(\square^{5}-\mathrm{C}_{9} \mathrm{H}_{5}-1,3-\left(\mathrm{SiMe}_{2}{ }^{\mathrm{t}} \mathrm{Bu}\right)_{2}\right) \mathrm{Zr}$ (3-THF). This molecule was prepared in a similar manner to $\mathbf{2}$ - THF with $0.132 \mathrm{~g}(0.17 \mathrm{mmol})$ of $\mathbf{3}$ yielding $0.048 \mathrm{~g}(33 \%)$ of bright red crystals identified as 3-THF. ${ }^{1} \mathrm{H}$ NMR (benzene- $\left.d_{6}\right)$ : $\square=-0.42\left(\mathrm{~s}, 6 \mathrm{H}, \mathrm{Si} M e_{2}{ }^{\mathrm{t}} \mathrm{Bu}\right), 0.00$ (s, 6H, SiMe $\left.{ }_{2}^{\mathrm{t}} \mathrm{Bu}\right), 0.28$ (s, 6H, SiMe $\left.{ }_{2}^{\mathrm{t}} \mathrm{Bu}\right), 0.65$ (s, 6H, $\mathrm{SiMe}{ }_{2}{ }^{\mathrm{t}} \mathrm{Bu}$ ), 0.93 (s, $\left.18 \mathrm{H}, \mathrm{SiMe}_{2}{ }^{t} \mathrm{Bu}\right), 1.16$ (s, 18H, $\left.\mathrm{SiMe}_{2}{ }^{t} \mathrm{Bu}\right), 2.31$ (br s, $4 \mathrm{H}, \mathrm{OCH}_{2} \mathrm{CH}_{2}$ ), 3.22 (s, 1H, Cp), 3.71 (m, 2H, Benzo), 6.12 (s, 1H, Cp), 6.78 (m, 2H, Benzo), 6.92 (m, 2H, Benzo), 7.97 ( $\mathrm{m}, 2 \mathrm{H}$, Benzo). One THF resonance not located. ${ }^{13} \mathrm{C}$ NMR (toluene- $d_{8}$, $\left.0{ }^{\circ} \mathrm{C}\right): \square=-5.09\left(\mathrm{SiMe}{ }_{2}{ }^{\mathrm{t}} \mathrm{Bu}\right),-4.37\left(\mathrm{Si} M e_{2}{ }^{\mathrm{t}} \mathrm{Bu}\right),-2.62\left(\mathrm{Si} M e_{2}{ }^{\mathrm{t}} \mathrm{Bu}\right),-1.73\left(\mathrm{Si} M e_{2}{ }^{\mathrm{t}} \mathrm{Bu}\right), 18.59$ $\left(\mathrm{SiMe}_{2} C \mathrm{Me}_{3}\right), 25.18\left(\mathrm{SiMe}_{2} C \mathrm{Me}_{3}\right), 27.63\left(\mathrm{SiMe}_{2} \mathrm{CMe} e_{3}\right), 27.80 \quad\left(\mathrm{SiMe}_{2} \mathrm{CMe} e_{3}\right), 47.42$ $\left(\mathrm{OCH}_{2} \mathrm{CH}_{2}\right), 74.93\left(\mathrm{OCH}_{2} \mathrm{CH}_{2}\right), 85.01,103.96,123.87$ (Benzo) 88.69, 116.04, 120.68 (Cp), 123.94, 123.96, 127.55 (Cp/Benzo). One Cp/Benzo resonance not located.

Preparation of rac/meso- $\left(\square^{6}-\mathrm{C}_{9} \mathrm{H}_{5}-1-\left(\mathrm{SiMe}_{3}\right)-3-\left(\mathrm{CMe}_{3}\right)\right)\left(\square^{5}-\mathrm{C}_{9} \mathrm{H}_{5}-1-\left(\mathrm{SiMe}_{3}\right)-3-\right.$ $\left.\left(\mathbf{C M e}_{3}\right)\right) \operatorname{Zr}(\mathbf{T H F})$ (4-THF). This molecule was prepared in a similar manner to 2-THF with $150 \mathrm{mg}(0.260 \mathrm{mmol})$ of 4 yielding $0.070 \mathrm{~g} \mathrm{(42 \% )}$ of bright red crystals identified as an approximately equimolar mixture of rac/meso-4-THF. Anal. Calcd. for $\mathrm{C}_{36} \mathrm{H}_{54} \mathrm{OSi}_{2} \mathrm{Zr}$ : C, 66.50; H, 8.37. Found: C, 66.28; H, 7.93. ${ }^{1} \mathrm{H}$ NMR (toluene- $\left.d_{8},-34{ }^{\circ} \mathrm{C}\right): \square=0.19(\mathrm{~s}$, 9H, SiMe $e_{3}$, 0.23 (s, 9H, SiMe $)_{3}, 0.28$ (s, 9H, SiMe $), 0.31$ (s, 9H, SiMe $e_{3}$ ), 0.64 (br s, 2H, $\mathrm{OCH}_{2} \mathrm{CH}_{2}$ ), 0.76 (br s, $2 \mathrm{H}, \mathrm{OCH}_{2} \mathrm{CH}_{2}$ ), 0.91 (br s, 2H, $\mathrm{OCH}_{2} \mathrm{CH}_{2}$ ), 1.19 (s, 9H, $\mathrm{CMe}_{3}$ ), $1.23\left(\mathrm{~s}, 9 \mathrm{H}, \mathrm{CMe}_{3}\right), 1.26\left(\mathrm{~s}, 9 \mathrm{H}, \mathrm{CMe} e_{3}\right), 1.27$ (s, 9H, CMe $\left.e_{3}\right), 2.02$ (br s, 2H, $\mathrm{OCH}_{2} \mathrm{CH}_{2}$ ), 2.19 (br s, $1 \mathrm{H}, \mathrm{OCH}_{2} \mathrm{CH}_{2}$ ), 2.30 (br s, $1 \mathrm{H}, \mathrm{OCH}_{2} \mathrm{CH}_{2}$ ), 2.42 (br s, $1 \mathrm{H}, \mathrm{OCH}_{2} \mathrm{CH}_{2}$ ), 2.48 (br 
s, $1 \mathrm{H}, \mathrm{OCH}_{2} \mathrm{CH}_{2}$ ), 2.74 (br s, $1 \mathrm{H}, \mathrm{OCH}_{2} \mathrm{CH}_{2}$ ), 2.90 (br s, $\left.1 \mathrm{H}, \mathrm{OCH}_{2} \mathrm{CH}_{2}\right), 3.42(\mathrm{~s}, 1 \mathrm{H}$, Cp), 3.45 (s, 1H, Cp), 3.65 (m, 2H, Benzo), 3.7 (br s, 2H, Benzo), 5.81 (s, 1H, Cp), 5.82 (s, 1H, Cp), 6.48 (d, 5 Hz, 1H, Benzo), 6.60 (d, 5 Hz, 1H, Benzo), 6.66 (d, 5 Hz, 1H, Benzo), 6.81 (br s, 6H, Benzo), 7.69 (d, 5 Hz, 1H, Benzo), 7.78 (br s, 1H, Benzo), 7.89 (d, $5 \mathrm{~Hz}, 1 \mathrm{H}$, Benzo). Two THF resonanes not located. ${ }^{13} \mathrm{C}$ NMR (toluene- $\left.d_{8},-34{ }^{\circ} \mathrm{C}\right): \square=$ $1.59\left(\mathrm{Si} M e_{3}\right), 2.08\left(\mathrm{Si} M e_{3}\right), 2.16\left(\mathrm{Si} M e_{3}\right), 24.45\left(\mathrm{OCH}_{2} \mathrm{CH}_{2}\right), 25.08\left(\mathrm{OCH}_{2} \mathrm{CH}_{2}\right), 26.15$ $\left(\mathrm{CMe}_{3}\right), 30.23\left(\mathrm{OCH}_{2} \mathrm{CH}_{2}\right), 32.69\left(\mathrm{CMe}_{3}\right), 32.91\left(\mathrm{CMe}_{3}\right), 33.08\left(\mathrm{CMe}_{3}\right) 33.33\left(\mathrm{CMe}_{3}\right)$, $33.59\left(\mathrm{CMe}_{3}\right), 33.81\left(\mathrm{CMe}_{3}\right), 33.95\left(\mathrm{CMe}_{3}\right), 44.08\left(\mathrm{OCH}_{2} \mathrm{CH}_{2}\right), 68.21\left(\mathrm{OCH}_{2} \mathrm{CH}_{2}\right), 73.35$ $\left(\mathrm{OCH}_{2} \mathrm{CH}_{2}\right), 83.94,84.99,85.58,86.05,86.28,101.25,101.32,102.08,102.18113 .92$, $114.03,114.60,115.29,116.31,118.53,119.28,119.60,120.87,122.94,123.74,123.89$, $124.62,124.34,126.10,126.26,126.46,126.57,127.12,127.81,127.99,131.63,133.37$, 135.86, 136.09 (Cp/Benzo). One $\mathrm{SiMe}_{3}$, two $\mathrm{Cp} /$ Benzo and two THF resonances not located.

\section{Preparation of $\left(\square^{6}-\mathrm{C}_{9} \mathrm{H}_{5}-1,3-\left(\mathrm{SiMe}_{3}\right)_{2}\right)\left(\square^{5}-\mathrm{C}_{9} \mathrm{H}_{5}-1,3-\left(\mathrm{SiMe}_{2} \mathrm{Ph}\right)_{2}\right) \mathrm{Zr}(\mathrm{THF})$ and $\left(\square^{6}-\right.$} $\left.\mathrm{C}_{9} \mathrm{H}_{5}-1,3-\left(\mathrm{SiMe}_{2} \mathrm{Ph}\right)_{2}\right)\left(\square^{5}-\mathrm{C}_{9} \mathrm{H}_{5}-1,3-\left(\mathrm{SiMe}_{3}\right)_{2}\right) \mathrm{Zr}(\mathrm{THF})$ (6-THF). This molecule was prepared in a similar manner to $\mathbf{2}$-THF with $0.214 \mathrm{~g}(0.29 \mathrm{mmol})$ of $\mathbf{6}$ and excess THF to yield $0.147 \mathrm{~g}(63 \%)$ of 6-THF as bright red crystals. The two haptomers are formed in an approximate 2:1 ratio. Where possible, the asterisks denote the major haptomer. ${ }^{1} \mathrm{H}$ NMR (benzene- $\left.d_{6}\right): \square=0.11\left(\mathrm{~s}, 36 \mathrm{H}, \mathrm{Si} M e_{3}\right), 0.26\left(\mathrm{~s}, 6 \mathrm{H}, \mathrm{Si} M e_{2} \mathrm{Ph}\right), 0.45$ (s, 6H, SiMe ${ }_{2} \mathrm{Ph}$ ), 0.49 (s, 6H, SiMe $2 \mathrm{Ph})^{*}, 0.64$ (br s, 4H, THF)*, 0.68 (br s, 4H, THF), 0.76 (s, 6H, $\left.\mathrm{Si}_{2} e_{2} \mathrm{Ph}\right)^{*}, 2.14$ (br s, 4H, THF)*, 2.27 (br s, 4H, THF), 3.06 (m, 2H, Benzo), 3.36 (s, $1 \mathrm{H}, \mathrm{Cp})^{*}, 3.40$ (s, 1H, Cp), $3.50(\mathrm{~m}, 2 \mathrm{H}, \mathrm{Benzo})^{*}, 6.31(\mathrm{~s}, 1 \mathrm{H}, \mathrm{Cp})^{*}, 6.32(\mathrm{~s}, 1 \mathrm{H}, \mathrm{Cp})$, 
$6.72(\mathrm{~m}, 2 \mathrm{H}, \text { Benzo })^{*}, 6.75(\mathrm{~m}, 2 \mathrm{H}$, Benzo), 7.00 (m, 2H, Benzo), 7.14-7.21 (m, 12H, $\mathrm{Ph}), 7.46(\mathrm{~m}, 4 \mathrm{H}, \mathrm{Ph}), 7.66(\mathrm{~d}, 8 \mathrm{~Hz}, 4 \mathrm{H}, \mathrm{Ph})^{*}, 7.75(\mathrm{~m}, 2 \mathrm{H}, \mathrm{Benzo})^{*}, 7.84(\mathrm{~m}, 2 \mathrm{H}$, Benzo)*, $7.91(\mathrm{~m}, 2 \mathrm{H}, \mathrm{Benzo}) .{ }^{13} \mathrm{C}$ NMR (benzene- $\left.d_{6}\right): \square=-0.61\left(\mathrm{Si} M e_{2} \mathrm{Ph}\right),-0.07$ (SiMe $2 \mathrm{Ph}), 1.12\left(\mathrm{Si} M e_{2} \mathrm{Ph}\right), 1.74$ (2 $\left.\mathrm{Si} M e_{3}\right), 24.29,24.47$ (THF), 73.49, 73.68 (THF), 86.20 (Benzo), 88.71 (Cp), 85.74, 86.33, 91.33, 103.31, 103.97, 115.78, 115.86, 117.62, $120.82,122.06,124.81,125.19,126.27,126.34,128.50,129.29,134.20,134.48,135.86$, 136.05, 138.36, 139.46, 139.59, 142.02, 142.92, 143.36 (Cp/Benzo/Ph). One $\mathrm{SiMe}_{2} \mathrm{Ph}$ resonance not located.

Preparation of $\left(\square^{6}-\mathrm{C}_{9} \mathrm{H}_{5}-1,3-\left(\mathrm{SiMe}_{3}\right)_{2}\right)\left(\square^{5}-\mathrm{C}_{9} \mathrm{H}_{5}-1-\left(\mathrm{SiMe}_{3}\right)-3-\left(\mathrm{CMe}_{3}\right)\right) \mathrm{Zr}(\mathrm{THF})$ and $\left(\square^{6}-\right.$ $\left.\mathrm{C}_{9} \mathrm{H}_{5}-1-\left(\mathrm{SiMe}_{3}\right)-3-\left(\mathrm{CMe}_{3}\right)\right)\left(\square^{5}-\mathrm{C}_{9} \mathrm{H}_{5}-1,3-\left(\mathrm{SiMe}_{3}\right)_{2}\right) \mathrm{Zr}(\mathrm{THF})$ (7-THF). This molecule was prepared in an indentical manner to 2-THF with $0.196 \mathrm{~g}(0.33 \mathrm{mmol})$ of 7 yielding 0.099 $\mathrm{g}(45 \%)$ of 7-THF as bright red crystals. The two haptomers are formed in an approximate 2:1 ratio. Where possible, asterisks denote the major haptomer. ${ }^{1} \mathrm{H}$ NMR (toluene- $d_{8},-58{ }^{\circ} \mathrm{C}$ ): $\square=0.18\left(\mathrm{~s}, 9 \mathrm{H}, \mathrm{Si} M e_{3}\right)^{*}, 0.20\left(\mathrm{~s}, 9 \mathrm{H}, \mathrm{Si}_{3}\right)^{*}, 0.25$ (s, 9H, SiMe $)_{3}$, 0.28 (s, 9H, SiMe $e_{3}$ ), 0.29 (s, 9H, SiMe $)_{3}^{*}, 0.37$ (s, 9H, SiMe ${ }_{3}$ ), 0.60 (br s, 2H, THF), 0.72 (br s, 2H, THF), 0.77 (br s, 2H, THF), 0.89 (br s, 2H, THF), 1.19 (s, 9H, CMe 3 ), 1.26 (s, 9H, $\mathrm{CMe}_{3}$ ), 1.96 (br s, 1H, THF)*, 2.16 (br s, 1H, THF)*, 2.30 (br s, 1H, THF), 2.43 (br s, 1H, THF)*, 2.53 (br s, 1H, THF), 2.78 (br s, 1H, THF)*, 3.37 (s, 1H, Cp)*, 3.41 (s, 1H, Cp), $3.63(\mathrm{~m}, 1 \mathrm{H}$, Benzo)*, 3.67 (m, 1H, Benzo)*, 3.73 (m, 1H, Benzo), 5.84 (s, 1H, Cp)*, 6.16 (s, 1H, Cp), 6.46 (d, 7 Hz, 1H, Benzo)*, 6.54 (d, 7 Hz, 1H, Benzo), 6.58 (d, 7 Hz, 1H, Benzo)*, 6.68 (d, 7 Hz, 1H, Benzo), 6.75 (d, 7 Hz, 1H, Benzo)*, 7.35 (d, 8 Hz, 1H, Benzo)*, 7.57 (d, $8 \mathrm{~Hz}, 1 \mathrm{H}$, Benzo $)^{*}, 7.72(\mathrm{~d}, 8 \mathrm{~Hz}, 1 \mathrm{H}$, Benzo), $7.82(\mathrm{~d}, 8 \mathrm{~Hz}, 1 \mathrm{H}$, 
Benzo), $7.84(\mathrm{~d}, 8 \mathrm{~Hz}, 1 \mathrm{H}$, Benzo)*. Two THF and three Benzo resonances from the minor isomer not located. ${ }^{13} \mathrm{C}$ NMR (toluene- $d_{8},-58{ }^{\circ} \mathrm{C}$ ): $\square=1.35,1.47^{*}, 1.60^{*}, 2.02$, 2.15*, $2.69\left(\mathrm{SiMe}_{3}\right), 24.47^{*}, 24.78,24.88,25.14 *(\mathrm{THF}), 32.80,33.33^{*}, 33.98^{*}, 34.66$ $\left(\mathrm{CMe}_{3}\right), 73.31^{*}, 74.56,74.71,75.82 *$ (THF), 83.96*, 85.49*, 85.96, 86.36 (Benzo), 90.05*, 90.97*, 93.43, 94.79, 101.36*, 101.5*, 103.42, 104.02, 114.74*, 114.84, 115.76*, $118.29^{*}, 118.53^{*}, 119.39,119.63^{*}, 120.16^{*}, 123.69,124.30,124.51,124.08,125.01$, $125.10^{*}, 125.16^{*}, 126.32,126.38^{*}, 126.54,126.67,132.80,134.67^{*}, 136.28^{*}, 136.62^{*}$, 140.36 (Cp/Benzo).

\section{Preparation of $\quad\left(\square^{6}-\mathrm{C}_{9} \mathrm{H}_{5}-1,3-\left(\mathrm{SiMe}_{2} \mathrm{Ph}\right)_{2}\right)\left(\square^{5}-\mathrm{C}_{9} \mathrm{H}_{5}-1,3-\left(\mathrm{SiMe}_{2} \mathrm{Ph}\right)_{2}\right) \mathrm{Zr}$} ( $\mathrm{MeOCH}_{2} \mathrm{CH}_{2} \mathrm{OMe}$ ) (2-DME). This molecule was prepared in an identical manner to 1DME with $0.125 \mathrm{~g}(0.146 \mathrm{mmol})$ of 2 and 90 torr $(0.161 \mathrm{mmol})$ of dimethoxyethane. Subsequent workup and recrystallization from pentane afforded $0.094 \mathrm{~g}(68 \%)$ of 2-DME as a green solid. Anal. Calcd. for $\mathrm{C}_{54} \mathrm{H}_{64} \mathrm{O}_{2} \mathrm{Si}_{4} \mathrm{Zr}$ : C, 68.39; H, 6.80. Found: C, 68.04; H, 6.46. ${ }^{1} \mathrm{H}$ NMR (benzene- $d_{6}$ ): $\mathrm{C}=0.31$ (s, $6 \mathrm{H}, \mathrm{SiMe} e_{2} \mathrm{Ph}$ ), 0.43 (s, $6 \mathrm{H}, \mathrm{SiMe} e_{2} \mathrm{Ph}$ ), 0.64 (s, 6H, SiMe $2 \mathrm{Ph}$ ), 0.75 (s, 6H, SiMe $2 \mathrm{Ph}$ ), $1.10\left(\mathrm{dd}, 2 \mathrm{H}, \mathrm{OCH}_{2}\right.$ ), 1.23 (dd, 2H, $\mathrm{OCH}_{2}$ ), 2.72 (s, 6H, OCH $\left.H_{3}\right), 3.65(\mathrm{~m}, 2 \mathrm{H}$, Benzo), $4.12(\mathrm{~s}, 1 \mathrm{H}, \mathrm{Cp}), 6.31(\mathrm{~m}, 2 \mathrm{H}, \mathrm{Benzo}), 6.69(\mathrm{~m}, 2 \mathrm{H}$, Benzo), 7.11 (s, 1H, Cp), 7.18 (m, 8H, Ph), 7.44 (m, 4H, Ph), 7.47 (m, 2H, Benzo), 7.64 $(\mathrm{m}, 8 \mathrm{H}, \mathrm{Ph}) .{ }^{13} \mathrm{C}$ NMR (benzene- $\left.d_{6}\right): \square=-0.14\left(\mathrm{Si} M e_{2} \mathrm{Ph}\right),-0.09\left(\mathrm{Si} M e_{2} \mathrm{Ph}\right),-0.06$ ( $\mathrm{SiMe} 2 \mathrm{Ph}), 2.14\left(\mathrm{SiMe} e_{2} \mathrm{Ph}\right), 67.56\left(\mathrm{OCH}_{2}\right), 68.43\left(\mathrm{OCH}_{3}\right), 83.01,83.33,105.39$ (Benzo) 107.37, 120.92 (Cp), 122.84, 123.69, 125.42, 129.62, 129.27, 134.33, 134.56, 137.02, 139.87, 142.60, 143.93 (Cp/Benzo/Ph). Two $\mathrm{Cp} /$ Benzo/Ph resonances not located. 


\section{Solution Characterization of $\left(\square^{6}-\mathrm{C}_{9} \mathrm{H}_{5}-1,3-\left(\mathrm{CHMe}_{2}\right)_{2}\right)\left(\square^{5}-\mathrm{C}_{9} \mathrm{H}_{5}-1,3 \quad-\right.$}

$\left.\left(\mathrm{CHMe}_{2}\right)_{2}\right) \mathrm{Zr}\left(\mathrm{MeOCH}_{2} \mathrm{CH}_{2} \mathrm{OMe}\right)$ (5-DME). This molecule was prepared in an identical manner to 1-DME with $0.017 \mathrm{~g}(0.035 \mathrm{mmol})$ of $\mathbf{5}$ and $182 \square \mathrm{L}(1.75 \mathrm{mmol})$ of dimethoxyethane. ${ }^{1} \mathrm{H}$ NMR (benzene- $\left.d_{6}\right): \mathrm{C}=0.93\left(\mathrm{~d}, 18 \mathrm{~Hz}, 6 \mathrm{H}, \mathrm{CH} M e_{2}\right), 1.10(\mathrm{~d}, 18 \mathrm{~Hz}$, 6H, CHMe $), 1.24\left(\mathrm{~d}, 18 \mathrm{~Hz}, 6 \mathrm{H}, \mathrm{CH} M e_{2}\right), 1.37$ (d, 18Hz, 6H, CHMe $\left.e_{2}\right), 1.70(\mathrm{~m}, 2 \mathrm{H}$, $\left.\mathrm{OCH}_{2}\right), 1.88\left(\mathrm{~m}, 2 \mathrm{H}, \mathrm{OCH}_{2}\right), 2.54\left(\mathrm{~m}, 2 \mathrm{H}, \mathrm{CHMe}_{2}\right), 2.84(\mathrm{~m}, 2 \mathrm{H}, \mathrm{CHMe}$ ), $2.93(\mathrm{~s}, 6 \mathrm{H}$, $\left.\mathrm{OCH}_{3}\right), 3.77$ (m, 2H, Benzo), 3.89 (s, 1H, Cp), 5.81 (s, 1H, Cp), 5.90 (m, 2H, Benzo), 6.79 (m, 2H, Benzo), 7.46 (m, 2H, Benzo).

\section{Preparation of $\left(\square^{5}-\mathrm{C}_{9} \mathrm{H}_{5}-1,3-\left(\mathrm{SiMe}_{3}\right)_{2}\right)\left(\square^{6}-\mathrm{C}_{9} \mathrm{H}_{5}-1,3-\left(\mathrm{SiMe}_{3}\right)_{2}\right) \mathrm{Zr}\left(\mathrm{EtOCH}_{2} \mathrm{CH}_{2} \mathrm{OEt}\right)(1-$}

DEE). This molecule was prepared in a similar manner as 1-DME using $0.05 \mathrm{~g}(0.08$ mmol) of 1 and 151 Torr $(0.8 \mathrm{mmol})$ of diethoxyethane from a $100.1 \mathrm{~mL}$ calibrated gas bulb, yielding $0.034 \mathrm{~g}(58 \%)$ of 1-DEE as a forest green solid upon recrystalization from pentane. Anal. Calcd. For $\mathrm{C}_{36} \mathrm{H}_{60} \mathrm{Si}_{4} \mathrm{O}_{2} \mathrm{Zr}$ : C, 59.36; H, 8.30. Found C, 59.09; H, 8.09. ${ }^{1} \mathrm{H}$ NMR (benzene- $\left.d_{6}\right): \square=0.17\left(\mathrm{~s}, 18 \mathrm{H}, \operatorname{Si} M e_{3}\right), 0.41\left(\mathrm{~s}, 18 \mathrm{H}, \mathrm{Si} M e_{3}\right), 0.79(\mathrm{t}, 7 \mathrm{~Hz}, 6 \mathrm{H}$, $\left.\mathrm{CH}_{2} \mathrm{CH}_{3}\right), 1.22\left(\mathrm{~m}, 2 \mathrm{H}, \mathrm{CH}_{2} \mathrm{CH}_{2}\right), 1.77\left(\mathrm{~m}, 2 \mathrm{H}, \mathrm{CH}_{2} \mathrm{CH}_{2}\right), 3.75\left(\mathrm{~m}, 2 \mathrm{H}, \mathrm{CH}_{2} \mathrm{CH}_{3}\right), 3.85$ (m, 2H, Benzo), 4.13 (s, 1H, Cp), $4.18\left(\mathrm{~m}, 2 \mathrm{H}, \mathrm{CH}_{2} \mathrm{CH}_{3}\right), 6.39$ (m, 2H, Benzo), 6.83 (m, 2H, Benzo), 6.88 (s, 1H, Cp), 7.59 (m, 2H, Benzo). ${ }^{13} \mathrm{C}$ NMR (benzene- $\left.d_{6}\right): \square=1.35,2.64$ $(\mathrm{SiMe}), 13.17\left(\mathrm{CH}_{2} \mathrm{CH}_{3}\right), 62.18\left(\mathrm{CH}_{2} \mathrm{CH}_{2}\right), 75.32\left(\mathrm{CH}_{2} \mathrm{CH}_{3}\right), 120.67,140.81(\mathrm{Cp}), 82.58$ 105.06, 123.39, 126.44 (Benzo), 84.92, 109.86, 121.56, 136.65 (Cp/Benzo).

Solution Characterization of $\left(\square^{6}-\mathrm{C}_{9} \mathrm{H}_{5}-1,3-\left(\mathrm{SiMe}_{3}\right)_{2}\right)\left(\square^{5}-\mathrm{C}_{9} \mathrm{H}_{5}-1,3-\right.$ $\left.\left(\mathrm{SiMe}_{3}\right)_{2}\right) \operatorname{Zr}\left(\mathrm{MeOCH}_{2} \mathbf{O M e}\right)$ (1-DMM). A J. Young NMR tube was charged with 0.023 
$\mathrm{mg}(0.040 \mathrm{mmol})$ of $\mathbf{1}$ and dissolved in approximately $0.5 \mathrm{~mL}$ of benzene- $d_{6}$. On the vacuum line, the solution was frozen in liquid nitrogen, degassed, and 47 torr $(0.08$ mmol) of dimethoxymethane was added by calibrated gas bulb. The tube was thawed, shaken, and the product characterized by NMR spectroscopy. Green 1-DMM can only be observed in the presence of a slight excess of dimethoxymethane. ${ }^{1} \mathrm{H}$ NMR (benzene- $d_{6}$ ): $\square=0.19$ (s, 18H, SiMe $), 0.37$ (s, 18H, SiMe $), 2.38$ (s, 6H, OCH $), 2.46$ (d, $4 \mathrm{~Hz}, 1 \mathrm{H}$, $\left.\mathrm{OCH}_{2}\right), 2.57\left(\mathrm{~d}, 4 \mathrm{~Hz}, 1 \mathrm{H}, \mathrm{OCH}_{2}\right), 3.67$ (m, 2H, Benzo), 3.84 (s, 1H, Cp), 6.58 (m, 2H, Benzo), 6.70 (s, 1H, Cp), 6.80 (m, 2H, Benzo), 7.69 (m, 2H, Benzo). ${ }^{13} \mathrm{C}$ NMR (benzene$\left.\left.d_{6}\right): \square=1.23(\mathrm{SiMe})_{3}\right), 2.30\left(\mathrm{Si} M e_{3}\right), 58.09\left(\mathrm{OCH}_{3}\right), 106.04\left(\mathrm{OCH}_{2}\right), 118.59,122.81$, 140.62 (Cp), 84.14, 104.39, 123.62, 126.39 (Benzo) 87.72, 111.18, 136.87 (Cp/Benzo).

\section{Solution Characterization of $\quad\left(\square^{6}-\mathrm{C}_{9} \mathrm{H}_{5}-1,3-\left(\mathrm{SiMe}_{3}\right)_{2}\right)\left(\square^{5}-\mathrm{C}_{9} \mathrm{H}_{5}-1,3-\right.$} $\left.\left(\mathrm{SiMe}_{3}\right)_{2}\right) \operatorname{Zr}\left(\mathrm{EtOCH}_{2} \mathrm{OEt}\right)$ (1-DEM). This molecule was observed in a similar manner to 1 -DMM using $0.017 \mathrm{~g}(0.028 \mathrm{mmol})$ of 1 and 517 torr $(2.8 \mathrm{mmol})$ of diethoxymethane added via a $100.1 \mathrm{~mL}$ calibrated gas bulb. Red 1-DEM can only be observed in the presence of a large excess of diethoxymethane. ${ }^{1} \mathrm{H}$ NMR (benzene- $\left.d_{6}\right): \square=0.17(\mathrm{~s}, 18 \mathrm{H}$, $\left.\mathrm{Si} M e_{3}\right), 0.33\left(\mathrm{~s}, 18 \mathrm{H}, \mathrm{Si} M e_{3}\right), 2.21\left(\mathrm{~d}, 4 \mathrm{~Hz}, \mathrm{OCH}_{2} \mathrm{O}\right), 3.38\left(\mathrm{OCH}_{2} \mathrm{O}\right)^{*}, 3.69(\mathrm{~m}, 2 \mathrm{H}$, Benzo), 3.89 (s, 1H, Cp), 6.49 (m, 2H, Benzo), 6.73 (s, 1H, Cp), 6.85 (m, 2H, Benzo), $7.65(\mathrm{~m}, 2 \mathrm{H}$, Benzo). Ethyl resonances from bound DEM were not located. * Denotes resonances that were obscured by free DEM and were identified by a g-COSY experiment. 
Solution Characterization of $\left(\square^{6}-\mathrm{C}_{9} \mathrm{H}_{5}-1,3-\left(\mathrm{SiMe}_{2} \mathrm{Ph}\right)_{2}\right)\left(\square^{5}-\mathrm{C}_{9} \mathrm{H}_{5}-1,3-\left(\mathrm{SiMe}_{2} \mathrm{Ph}\right)_{2}\right.$ ) $\mathrm{Zr}\left(\mathrm{MeOCH}_{2} \mathrm{OMe}\right)$ (2-DMM). This molecule was prepared in an identical manner to 1DMM with $0.020 \mathrm{~g}(0.023 \mathrm{mmol})$ of 2 and 41 torr $(0.069 \mathrm{mmol})$ of dimethoxymethane. Green 2-DMM can only be observed in the presence of a slight excess of dimethoxymethane. ${ }^{1} \mathrm{H}$ NMR (benzene- $\left.d_{6}\right): \square=0.24$ (s, 6H, SiMe $e_{2} \mathrm{Ph}$ ), 0.53 (s, 6H, $\mathrm{Si}_{2} \mathrm{Ph}$ ), 0.62 (s, 6H, SiMe $2 \mathrm{Ph}$ ), 0.65 (s, 6H, SiMe $2 \mathrm{Ph}$ ), 2.07 (s, 6H, $\mathrm{CH}_{3} \mathrm{O}$ ), 2.37 (d, $\left.5 \mathrm{~Hz}, 1 \mathrm{H}, \mathrm{OCH}_{2}\right), 2.42\left(\mathrm{~d}, 5 \mathrm{~Hz}, 1 \mathrm{H}, \mathrm{OCH}_{2}\right), 3.31(\mathrm{~m}, 2 \mathrm{H}, \mathrm{Benzo}), 3.84(\mathrm{~s}, 1 \mathrm{H}, \mathrm{Cp}), 6.57$ (m, 2H, Benzo), 6.63 (m, 2H, Benzo), 6.93 (s, 1H, Cp), 7.17 (m, 4H, Ph), 7.27 (t, 8Hz, 2H, Benzo), 7.53 (d, 8Hz, 8H, Ph), 7.58 (m, 2H, Benzo), 7.75 (d, 8Hz, 8H, Ph). ${ }^{13} \mathrm{C}$ NMR (benzene- $\left.d_{6}\right):-\left[=-0.34\left(\mathrm{Si} M e_{2} \mathrm{Ph}\right), 0.41\left(\mathrm{Si} M e_{2} \mathrm{Ph}\right), 0.74\left(\mathrm{Si} M e_{2} \mathrm{Ph}\right), 1.43\left(\mathrm{Si} M e_{2} \mathrm{Ph}\right)\right.$, $58.11\left(\mathrm{OCH}_{3}\right), 85.51$ (Benzo), $105.19\left(\mathrm{OCH}_{2}\right), 119.29,123.75(\mathrm{Cp}), 106.14,108.25$, $123.91,126.34,127.81,128.15,129.25,134.33,134.63,137.09,139.76,143.24,143.58$ $(\mathrm{Cp} / \mathrm{Benzo} / \mathrm{Ph})$. Two $\mathrm{Cp} /$ Benzo/Ph resonances not located.

\section{P r e p a r a i o n $\quad$ of $\left(\square^{5}-\mathrm{C}_{9} \mathrm{H}_{5}-1,3-\left(\mathrm{SiMe}_{3}\right)_{2}\right)\left(\square^{6}-\mathrm{C}_{9} \mathrm{H}_{5}-1,3-\left(\mathrm{SiMe}_{3}\right)_{2}\right) \mathrm{Zr}$} $\left(\mathrm{CH}_{3} \mathrm{OC}\left(\mathrm{CH}_{3}\right) \mathrm{HC}\left(\mathrm{CH}_{3}\right) \mathrm{OCH}_{3}\right)$ (1-rac-DMB). A J. Young NMR tube was charged with $0.06 \mathrm{~g}(0.1 \mathrm{mmol})$ of 1 and approximately $0.5 \mathrm{~mL}$ benzene. To the resulting solution was added $100 \square \mathrm{L}$ of a solution containing a 50:50 mixture of dimethoxybutane and THF. Both diastereomers of dimethoxybutane were present. The resulting reaction mixture was shaken for 15 minutes. The solvent was then removed from the resulting green solution and this procedure was repeated twice to enrich the sample with 1-DMB. Removal of the solvent after the third cycle and recrystallization from pentane at $-35^{\circ} \mathrm{C}$ afforded $0.030 \mathrm{~g}$ (42\%) of 1-rac-DMB free of 1-meso-DMB. Anal. Calcd. For $\mathrm{C}_{36} \mathrm{H}_{50} \mathrm{Si}_{4} \mathrm{O}_{2} \mathrm{Zr}$ : C, 59.36; 
$\mathrm{H}, 8.30$. Found $\mathrm{C}, 59.51 ; \mathrm{H}, 7.92 .{ }^{1} \mathrm{H}$ NMR (benzene- $\left.d_{6}\right): \square=0.06\left(\mathrm{~m}, 6 \mathrm{H}, \mathrm{CHCH}_{3}\right), 0.18$ (s, 9H, SiMe $), 0.20$ (s, 9H, SiMe $), 0.40$ (s, 9H, SiMe $), 0.41$ (s, 9H, SiMe $), 1.68$ (m, 1H, $\left.\mathrm{CHCH}_{3}\right), 1.80\left(\mathrm{~m}, 1 \mathrm{H}, \mathrm{CHCH}_{3}\right), \quad(\mathrm{s}, 1 \mathrm{H}, \mathrm{Cp}), 3.34\left(\mathrm{~s}, 3 \mathrm{H}, \mathrm{OCH}_{3}\right), 3.43\left(\mathrm{~s}, 3 \mathrm{H}, \mathrm{OCH}_{3}\right)$, 3.89 (m, 2H, Benzo), 4.16 (s, 1H, Cp), 6.33 (m, 1H, Benzo), 6.44 (m, 1H, Benzo), 6.79 (s, 1H, Cp), 6.86 (m, 2H, Benzo), 7.59 (m, 1H, Benzo), 7.65 (m, 1H, Benzo). ${ }^{13} \mathrm{C}$ NMR (benzene- $\left.d_{6}\right): \square=\left(\operatorname{Si} M e_{3}\right),\left(\right.$ Benzo). ${ }^{13} \mathrm{C}$ NMR (benzene- $\left.d_{6}\right): \square=1.40,1.66,2.51,2.90$ $\left(\mathrm{SiMe}_{3}\right), 14.69,15.89\left(\mathrm{CHCH}_{3}\right), 63.69,66.07,75.15,78.55\left(\mathrm{OCHCH}_{3} / \mathrm{OCH}_{3}\right), 82.87$, $83.52,85.02,86.02,104.85,105.26,108.65,121.39,123.87,125.16,125.75,126.08$, 135.97, 140.39 (Cp/Benzo). Three Cp/Benzo resonances not located.

\section{P r e p a r a i o n $\quad$ of $\left(\square^{6}-\mathrm{C}_{9} \mathrm{H}_{5}-1,3-\left(\mathrm{SiMe}_{3}\right)_{2}\right)\left(\square^{5}-\mathrm{C}_{9} \mathrm{H}_{5}-1,3-\left(\mathrm{SiMe}_{3}\right)_{2}\right) \mathrm{Zr}$} $\left(\mathrm{CH}_{3} \mathrm{OC}\left(\mathrm{CH}_{3}\right) \mathrm{HC}\left(\mathrm{CH}_{3}\right) \mathrm{OCH}_{3}\right)$ (meso-1-DMB). This molecule can be observed in a similar manner as rac-1-DMB using $0.02 \mathrm{~g}(0.03 \mathrm{mmol})$ of $\mathbf{1}$ and $45 \square \mathrm{L}(0.38 \mathrm{mmol})$ of diasteromerically pure meso-dimethoxybutane. The desired product can only be observed in a large excess of meso-DMB. ${ }^{1} \mathrm{H}$ NMR (benzene- $d_{6}$ ): $\square=0.30\left(\mathrm{~s}, 18 \mathrm{H}, \mathrm{Si} M e_{3}\right.$ ), 0.42 (s, 18H, SiMe $\left.e_{3}\right), 1.63\left(\mathrm{~m}, 1 \mathrm{H}, \mathrm{CH}\left(\mathrm{CH}_{3}\right)\right), 3.31\left(\mathrm{~s}, 3 \mathrm{H}, \mathrm{OCH}_{3}\right) 3.38\left(\mathrm{~s}, 3 \mathrm{H}, \mathrm{OCH}_{3}\right), 3.90(\mathrm{~m}$, 2H, Benzo), $4.21(\mathrm{~s}, 1 \mathrm{H}, \mathrm{Cp}), 6.43(\mathrm{~m}, 2 \mathrm{H}$, Benzo), $6.90(\mathrm{~s}, 1 \mathrm{H}, \mathrm{Cp}), 6.95(\mathrm{~m}, 2 \mathrm{H}$, Benzo), 7.59 ( $\mathrm{m}, 2 \mathrm{H}$, Benzo). Two $\mathrm{CH}\left(\mathrm{CH}_{3}\right)$ and one $\mathrm{CH}\left(\mathrm{CH}_{3}\right)$ resonances not located. Integration of the ${ }^{1} \mathrm{H}$ NMR spectrum indicated a 2:1 mixture of two isomers.

\section{Solution Characterization of $\left(\square^{6}-\mathrm{C}_{9} \mathrm{H}_{5}-1,3-\left(\mathrm{SiMe}_{3}\right)_{2}\right)\left(\square^{5}-\mathrm{C}_{9} \mathrm{H}_{5}-1,3-\left(\mathrm{SiMe}_{3}\right)_{2}\right) \mathrm{Zr}$} $\left(\mathrm{CH}_{3} \mathrm{OCH}_{3}\right)(\mathbf{1 - O M e})$. Observed in a procedure similar for that of 1-DMM using 0.020 $\mathrm{g}(0.033 \mathrm{mmol})$ of $\mathbf{1}$ and 314 Torr $(1.7 \mathrm{mmol})$ of dimethyl ether from a $100.1 \mathrm{~mL}$ calibrated gas volume. Conversion to $\mathbf{1 - O M e _ { 2 }}$ occurs over the course of minutes at 
ambient temperature. Red 1-OMe $\mathbf{M}_{2}$ can only be observed in the presence of excess dimethyl ether. ${ }^{1} \mathrm{H}$ NMR (benzene- $d_{6}$ ): $\square=0.11$ (s, 36H, SiMe 3 ), 1.51 (br s, 6H, MeOMe), 3.36 (s, 1H, Cp), 3.55 (t, 4Hz, 2H, Benzo), 6.26 (s, 1H, Cp), 6.77 (t, 4Hz, 2H, Benzo), 6.89 (m, 2H, Benzo), 7.86 (m, 2H, Benzo). 


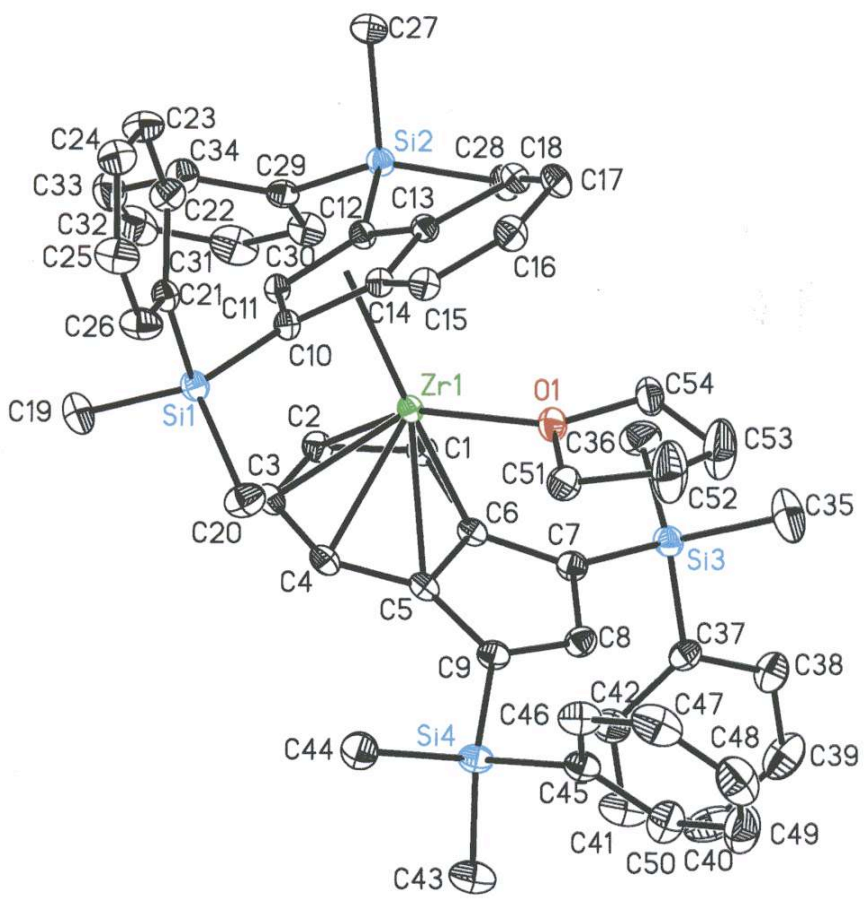

Figure S4. Fully labeled view of the molecular structure of $\mathbf{2 - T H F}$ at $30 \%$ probability ellipsoids with hydrogen atoms omitted for clarity. 
Table S2. Crystal data and structure refinement for 2-THF.

Identification code

Empirical formula

Formula weight

Temperature

Wavelength

Crystal system

Space group

Unit cell dimensions

Volume

Z

Density (calculated)

Absorption coefficient

$\mathrm{F}(000)$

Crystal size

Theta range for data collection

Index ranges

Reflections collected

Independent reflections

Completeness to theta $=36.14^{\circ}$

Absorption correction

Max. and min. transmission

Refinement method

Data / restraints / parameters

Goodness-of-fit on $\mathrm{F}^{2}$

Final $\mathrm{R}$ indices $[\mathrm{I}>2 \operatorname{sigma}(\mathrm{I})]$

$\mathrm{R}$ indices (all data)

Largest diff. peak and hole
2-THF

C54 H62 O Si4 Zr

930.62

173(2) K

$0.71073 \AA$

Triclinic

P-1

$\begin{array}{ll}\mathrm{a}=12.7495(10) \AA & \square=87.677(2)^{\circ} . \\ \mathrm{b}=12.9326(10) \AA & \square=70.785(2)^{\circ} . \\ \mathrm{c}=17.4158(13) \AA & \square=64.140(2)^{\circ} .\end{array}$

2422.1(3) $\AA^{3}$

2

$1.276 \mathrm{Mg} / \mathrm{m}^{3}$

$0.364 \mathrm{~mm}^{-1}$

980

$0.40 \times 0.30 \times 0.25 \mathrm{~mm}^{3}$

2.28 to $36.14^{\circ}$.

$-18<=\mathrm{h}<=18,-18<=\mathrm{k}<=18,-24<=\mathrm{l}<=28$

31134

$15284[\mathrm{R}($ int $)=0.0307]$

$65.9 \%$

\section{SADABS}

0.9146 and 0.8682

Full-matrix least-squares on $\mathrm{F}^{2}$

15284 / 0 / 773

1.034

$\mathrm{R} 1=0.0402, \mathrm{wR} 2=0.0919$

$\mathrm{R} 1=0.0609, \mathrm{wR} 2=0.1001$

0.830 and -0.722 e. $\AA^{-3}$ 
Table S3. Bond lengths $[\AA]$ and angles $\left[{ }^{\circ}\right]$ for

2-THF.

\begin{tabular}{|c|c|}
\hline $\mathrm{Zr}(1)-\mathrm{O}(1)$ & $2.2304(12)$ \\
\hline $\operatorname{Zr}(1)-C(14)$ & $2.3540(17)$ \\
\hline $\operatorname{Zr}(1)-\mathrm{C}(11)$ & $2.3573(17)$ \\
\hline $\operatorname{Zr}(1)-C(3)$ & $2.4396(17)$ \\
\hline $\operatorname{Zr}(1)-\mathrm{C}(12)$ & $2.4776(17)$ \\
\hline $\operatorname{Zr}(1)-C(13)$ & $2.4796(17)$ \\
\hline $\operatorname{Zr}(1)-C(15)$ & $2.4882(17)$ \\
\hline $\operatorname{Zr}(1)-C(4)$ & $2.4906(17)$ \\
\hline $\operatorname{Zr}(1)-C(10)$ & $2.4914(17)$ \\
\hline $\operatorname{Zr}(1)-C(2)$ & $2.4913(17)$ \\
\hline $\operatorname{Zr}(1)-C(1)$ & $2.6227(17)$ \\
\hline $\operatorname{Zr}(1)-C(5)$ & $2.6237(17)$ \\
\hline $\mathrm{Si}(1)-\mathrm{C}(20)$ & $1.861(2)$ \\
\hline $\operatorname{Si}(1)-C(2)$ & $1.8632(17)$ \\
\hline $\operatorname{Si}(1)-C(19)$ & $1.867(2)$ \\
\hline $\operatorname{Si}(1)-C(21)$ & $1.8793(19)$ \\
\hline $\mathrm{Si}(2)-\mathrm{C}(4)$ & $1.8646(17)$ \\
\hline $\operatorname{Si}(2)-C(27)$ & $1.866(2)$ \\
\hline $\operatorname{Si}(2)-C(28)$ & $1.870(2)$ \\
\hline $\operatorname{Si}(2)-C(29)$ & $1.8716(18)$ \\
\hline $\mathrm{Si}(3)-\mathrm{C}(16)$ & $1.8492(18)$ \\
\hline $\operatorname{Si}(3)-C(35)$ & $1.868(3)$ \\
\hline $\mathrm{Si}(3)-\mathrm{C}(36)$ & $1.870(3)$ \\
\hline $\operatorname{Si}(3)-C(37)$ & $1.880(2)$ \\
\hline $\mathrm{Si}(4)-\mathrm{C}(18)$ & $1.8602(18)$ \\
\hline $\operatorname{Si}(4)-C(43)$ & $1.864(3)$ \\
\hline $\mathrm{Si}(4)-\mathrm{C}(44)$ & $1.867(2)$ \\
\hline $\operatorname{Si}(4)-C(45)$ & $1.890(2)$ \\
\hline $\mathrm{O}(1)-\mathrm{C}(51)$ & $1.467(2)$ \\
\hline $\mathrm{O}(1)-\mathrm{C}(54)$ & $1.470(2)$ \\
\hline C(1)-C(9) & $1.420(2)$ \\
\hline $\mathrm{C}(1)-\mathrm{C}(5)$ & $1.431(2)$ \\
\hline
\end{tabular}

\begin{tabular}{|c|c|}
\hline $\mathrm{C}(1)-\mathrm{C}(2)$ & $1.451(2)$ \\
\hline$C(2)-C(3)$ & $1.435(2)$ \\
\hline$C(3)-C(4)$ & $1.431(2)$ \\
\hline$C(4)-C(5)$ & $1.456(2)$ \\
\hline$C(5)-C(6)$ & $1.419(2)$ \\
\hline$C(6)-C(7)$ & $1.371(3)$ \\
\hline$C(7)-C(8)$ & $1.414(3)$ \\
\hline$C(8)-C(9)$ & $1.370(3)$ \\
\hline$C(10)-C(18)$ & $1.427(2)$ \\
\hline$C(10)-C(15)$ & $1.448(2)$ \\
\hline$C(10)-C(11)$ & $1.450(2)$ \\
\hline$C(11)-C(12)$ & $1.426(3)$ \\
\hline$C(12)-C(13)$ & $1.394(3)$ \\
\hline$C(13)-C(14)$ & $1.425(2)$ \\
\hline$C(14)-C(15)$ & $1.452(2)$ \\
\hline$C(15)-C(16)$ & $1.432(2)$ \\
\hline$C(16)-C(17)$ & $1.416(3)$ \\
\hline$C(17)-C(18)$ & $1.412(3)$ \\
\hline$C(21)-C(22)$ & $1.394(3)$ \\
\hline$C(21)-C(26)$ & $1.398(3)$ \\
\hline$C(22)-C(23)$ & $1.381(3)$ \\
\hline$C(23)-C(24)$ & $1.384(3)$ \\
\hline$C(24)-C(25)$ & $1.372(3)$ \\
\hline$C(25)-C(26)$ & $1.388(3)$ \\
\hline$C(29)-C(30)$ & $1.395(3)$ \\
\hline$C(29)-C(34)$ & $1.397(3)$ \\
\hline$C(30)-C(31)$ & $1.387(3)$ \\
\hline$C(31)-C(32)$ & $1.377(4)$ \\
\hline$C(32)-C(33)$ & $1.382(4)$ \\
\hline$C(33)-C(34)$ & $1.389(3)$ \\
\hline $\mathrm{C}(37)-\mathrm{C}(38)$ & $1.393(3)$ \\
\hline$C(37)-C(42)$ & $1.401(3)$ \\
\hline$C(38)-C(39)$ & $1.388(3)$ \\
\hline$C(39)-C(40)$ & $1.367(4)$ \\
\hline$C(40)-C(41)$ & $1.377(4)$ \\
\hline
\end{tabular}




\begin{tabular}{|c|c|c|c|}
\hline $\mathrm{C}(41)-\mathrm{C}(42)$ & $1.384(3)$ & $\mathrm{C}(12)-\operatorname{Zr}(1)-\mathrm{C}(4)$ & $102.64(6)$ \\
\hline $\mathrm{C}(45)-\mathrm{C}(50)$ & $1.394(3)$ & $C(13)-\operatorname{Zr}(1)-C(4)$ & $87.39(6)$ \\
\hline$C(45)-C(46)$ & $1.396(3)$ & $\mathrm{C}(15)-\operatorname{Zr}(1)-\mathrm{C}(4)$ & $130.09(5)$ \\
\hline $\mathrm{C}(46)-\mathrm{C}(47)$ & $1.386(4)$ & $\mathrm{O}(1)-\operatorname{Zr}(1)-\mathrm{C}(10)$ & $87.64(5)$ \\
\hline C(47)-C(48) & $1.371(4)$ & $\mathrm{C}(14)-\mathrm{Zr}(1)-\mathrm{C}(10)$ & $62.48(6)$ \\
\hline C(48)-C(49) & $1.362(5)$ & $\mathrm{C}(11)-\operatorname{Zr}(1)-\mathrm{C}(10)$ & $34.66(6)$ \\
\hline $\mathrm{C}(49)-\mathrm{C}(50)$ & $1.392(4)$ & C(3)-Zr(1)-C(10) & $136.21(6)$ \\
\hline$C(51)-C(52)$ & $1.491(3)$ & $C(12)-\operatorname{Zr}(1)-C(10)$ & $58.68(6)$ \\
\hline $\mathrm{C}(52)-\mathrm{C}(53)$ & $1.452(4)$ & $\mathrm{C}(13)-\mathrm{Zr}(1)-\mathrm{C}(10)$ & $69.20(6)$ \\
\hline \multirow[t]{2}{*}{$C(53)-C(54)$} & $1.480(3)$ & $C(15)-\operatorname{Zr}(1)-C(10)$ & $33.82(5)$ \\
\hline & & $\mathrm{C}(4)-\operatorname{Zr}(1)-\mathrm{C}(10)$ & $156.46(6)$ \\
\hline $\mathrm{O}(1)-\mathrm{Zr}(1)-\mathrm{C}(14)$ & $113.54(6)$ & $\mathrm{O}(1)-\mathrm{Zr}(1)-\mathrm{C}(2)$ & $109.29(5)$ \\
\hline $\mathrm{O}(1)-\mathrm{Zr}(1)-\mathrm{C}(11)$ & $111.88(6)$ & $C(14)-\operatorname{Zr}(1)-C(2)$ & $136.09(6)$ \\
\hline$C(14)-\operatorname{Zr}(1)-C(11)$ & $76.12(6)$ & $\mathrm{C}(11)-\operatorname{Zr}(1)-\mathrm{C}(2)$ & $96.83(6)$ \\
\hline $\mathrm{O}(1)-\operatorname{Zr}(1)-\mathrm{C}(3)$ & $132.54(5)$ & $C(3)-\operatorname{Zr}(1)-C(2)$ & $33.82(5)$ \\
\hline$C(14)-\operatorname{Zr}(1)-C(3)$ & $104.75(6)$ & $\mathrm{C}(12)-\operatorname{Zr}(1)-\mathrm{C}(2)$ & $88.47(6)$ \\
\hline $\mathrm{C}(11)-\mathrm{Zr}(1)-\mathrm{C}(3)$ & $103.47(6)$ & $C(13)-\operatorname{Zr}(1)-C(2)$ & $104.34(6)$ \\
\hline $\mathrm{O}(1)-\mathrm{Zr}(1)-\mathrm{C}(12)$ & $145.10(6)$ & $\mathrm{C}(15)-\operatorname{Zr}(1)-\mathrm{C}(2)$ & $157.16(6)$ \\
\hline$C(14)-\operatorname{Zr}(1)-C(12)$ & $61.26(6)$ & $C(4)-\operatorname{Zr}(1)-C(2)$ & $57.24(6)$ \\
\hline$C(11)-\operatorname{Zr}(1)-C(12)$ & $34.19(6)$ & $\mathrm{C}(10)-\operatorname{Zr}(1)-\mathrm{C}(2)$ & $129.59(6)$ \\
\hline$C(3)-\operatorname{Zr}(1)-C(12)$ & $78.18(6)$ & $\mathrm{O}(1)-\mathrm{Zr}(1)-\mathrm{C}(1)$ & $80.55(5)$ \\
\hline $\mathrm{O}(1)-\mathrm{Zr}(1)-\mathrm{C}(13)$ & $146.32(6)$ & $\mathrm{C}(14)-\operatorname{Zr}(1)-\mathrm{C}(1)$ & $151.43(6)$ \\
\hline$C(14)-\operatorname{Zr}(1)-C(13)$ & $34.17(6)$ & $\mathrm{C}(11)-\operatorname{Zr}(1)-\mathrm{C}(1)$ & $123.25(6)$ \\
\hline$C(11)-\operatorname{Zr}(1)-C(13)$ & $61.30(6)$ & $C(3)-Z r(1)-C(1)$ & $53.33(5)$ \\
\hline$C(3)-\operatorname{Zr}(1)-C(13)$ & $78.75(6)$ & $\mathrm{C}(12)-\operatorname{Zr}(1)-\mathrm{C}(1)$ & $121.30(6)$ \\
\hline $\mathrm{C}(12)-\operatorname{Zr}(1)-\mathrm{C}(13)$ & $32.67(6)$ & $\mathrm{C}(13)-\operatorname{Zr}(1)-\mathrm{C}(1)$ & $132.06(6)$ \\
\hline $\mathrm{O}(1)-\mathrm{Zr}(1)-\mathrm{C}(15)$ & $88.39(5)$ & $\mathrm{C}(15)-\operatorname{Zr}(1)-\mathrm{C}(1)$ & $168.86(5)$ \\
\hline$C(14)-\operatorname{Zr}(1)-C(15)$ & $34.77(6)$ & $C(4)-Z r(1)-C(1)$ & $54.67(5)$ \\
\hline$C(11)-\operatorname{Zr}(1)-C(15)$ & $62.31(6)$ & $\mathrm{C}(10)-\operatorname{Zr}(1)-\mathrm{C}(1)$ & $145.54(5)$ \\
\hline$C(3)-\operatorname{Zr}(1)-C(15)$ & $137.10(6)$ & $C(2)-Z r(1)-C(1)$ & $32.83(5)$ \\
\hline$C(12)-\operatorname{Zr}(1)-C(15)$ & $69.08(6)$ & $\mathrm{O}(1)-\mathrm{Zr}(1)-\mathrm{C}(5)$ & $82.05(5)$ \\
\hline $\mathrm{C}(13)-\operatorname{Zr}(1)-\mathrm{C}(15)$ & $58.69(6)$ & $C(14)-\operatorname{Zr}(1)-C(5)$ & $122.43(6)$ \\
\hline $\mathrm{O}(1)-\mathrm{Zr}(1)-\mathrm{C}(4)$ & $112.25(5)$ & $\mathrm{C}(11)-\operatorname{Zr}(1)-\mathrm{C}(5)$ & $151.47(6)$ \\
\hline$C(14)-Z r(1)-C(4)$ & $96.80(6)$ & $C(3)-Z r(1)-C(5)$ & $53.37(5)$ \\
\hline$C(11)-Z r(1)-C(4)$ & $134.25(6)$ & $C(12)-\operatorname{Zr}(1)-C(5)$ & $131.36(6)$ \\
\hline$C(3)-\operatorname{Zr}(1)-C(4)$ & $33.74(5)$ & $\mathrm{C}(13)-\operatorname{Zr}(1)-\mathrm{C}(5)$ & $120.30(6)$ \\
\hline
\end{tabular}




\begin{tabular}{|c|c|c|c|}
\hline$C(15)-\operatorname{Zr}(1)-C(5)$ & $145.41(5)$ & $C(5)-C(1)-\operatorname{Zr}(1)$ & $74.21(10)$ \\
\hline$C(4)-\operatorname{Zr}(1)-C(5)$ & $32.96(5)$ & $C(2)-C(1)-\operatorname{Zr}(1)$ & $68.60(9)$ \\
\hline$C(10)-\operatorname{Zr}(1)-C(5)$ & $169.68(5)$ & $\mathrm{C}(3)-\mathrm{C}(2)-\mathrm{C}(1)$ & $104.26(14)$ \\
\hline $\mathrm{C}(2)-\operatorname{Zr}(1)-\mathrm{C}(5)$ & $54.65(5)$ & $\mathrm{C}(3)-\mathrm{C}(2)-\mathrm{Si}(1)$ & $130.36(12)$ \\
\hline$C(1)-\operatorname{Zr}(1)-C(5)$ & $31.65(5)$ & $C(1)-C(2)-S i(1)$ & $123.96(12)$ \\
\hline $\mathrm{C}(20)-\mathrm{Si}(1)-\mathrm{C}(2)$ & $112.31(10)$ & $\mathrm{C}(3)-\mathrm{C}(2)-\operatorname{Zr}(1)$ & $71.12(9)$ \\
\hline$C(20)-\operatorname{Si}(1)-C(19)$ & $110.59(12)$ & $C(1)-C(2)-\operatorname{Zr}(1)$ & $78.57(9)$ \\
\hline$C(2)-\operatorname{Si}(1)-C(19)$ & $110.10(9)$ & $\mathrm{Si}(1)-\mathrm{C}(2)-\operatorname{Zr}(1)$ & $125.65(9)$ \\
\hline$C(20)-\operatorname{Si}(1)-C(21)$ & $108.72(10)$ & $C(4)-C(3)-C(2)$ & $112.73(14)$ \\
\hline$C(2)-S i(1)-C(21)$ & $105.86(8)$ & $C(4)-C(3)-\operatorname{Zr}(1)$ & $75.09(9)$ \\
\hline $\mathrm{C}(19)-\mathrm{Si}(1)-\mathrm{C}(21)$ & $109.10(10)$ & $C(2)-C(3)-\operatorname{Zr}(1)$ & $75.07(10)$ \\
\hline$C(4)-S i(2)-C(27)$ & $109.08(9)$ & $\mathrm{C}(3)-\mathrm{C}(4)-\mathrm{C}(5)$ & $104.28(14)$ \\
\hline C(4)-Si(2)-C(28) & $111.63(10)$ & $C(3)-C(4)-S i(2)$ & $129.06(13)$ \\
\hline $\mathrm{C}(27)-\mathrm{Si}(2)-\mathrm{C}(28)$ & 107.91(11) & $C(5)-C(4)-S i(2)$ & $125.19(12)$ \\
\hline $\mathrm{C}(4)-\mathrm{Si}(2)-\mathrm{C}(29)$ & $110.56(8)$ & $C(3)-C(4)-\operatorname{Zr}(1)$ & $71.18(9)$ \\
\hline $\mathrm{C}(27)-\mathrm{Si}(2)-\mathrm{C}(29)$ & $108.05(9)$ & $C(5)-C(4)-\operatorname{Zr}(1)$ & $78.55(10)$ \\
\hline $\mathrm{C}(28)-\mathrm{Si}(2)-\mathrm{C}(29)$ & $109.51(10)$ & $\mathrm{Si}(2)-\mathrm{C}(4)-\operatorname{Zr}(1)$ & $125.70(8)$ \\
\hline $\mathrm{C}(16)-\mathrm{Si}(3)-\mathrm{C}(35)$ & $114.57(10)$ & $C(6)-C(5)-C(1)$ & $119.50(16)$ \\
\hline $\mathrm{C}(16)-\mathrm{Si}(3)-\mathrm{C}(36)$ & $110.90(12)$ & $C(6)-C(5)-C(4)$ & $131.44(16)$ \\
\hline $\mathrm{C}(35)-\mathrm{Si}(3)-\mathrm{C}(36)$ & $108.22(17)$ & $C(1)-C(5)-C(4)$ & $109.04(14)$ \\
\hline $\mathrm{C}(16)-\mathrm{Si}(3)-\mathrm{C}(37)$ & $108.72(8)$ & $C(6)-C(5)-\operatorname{Zr}(1)$ & $124.72(12)$ \\
\hline $\mathrm{C}(35)-\mathrm{Si}(3)-\mathrm{C}(37)$ & $107.46(12)$ & $C(1)-C(5)-\operatorname{Zr}(1)$ & $74.14(10)$ \\
\hline $\mathrm{C}(36)-\mathrm{Si}(3)-\mathrm{C}(37)$ & $106.63(13)$ & $C(4)-C(5)-\operatorname{Zr}(1)$ & $68.49(9)$ \\
\hline $\mathrm{C}(18)-\mathrm{Si}(4)-\mathrm{C}(43)$ & $110.02(11)$ & $C(7)-C(6)-C(5)$ & $119.15(18)$ \\
\hline $\mathrm{C}(18)-\mathrm{Si}(4)-\mathrm{C}(44)$ & $113.80(10)$ & $C(6)-C(7)-C(8)$ & $121.27(17)$ \\
\hline C(43)-Si(4)-C(44) & $107.66(14)$ & $\mathrm{C}(9)-\mathrm{C}(8)-\mathrm{C}(7)$ & $121.20(18)$ \\
\hline $\mathrm{C}(18)-\mathrm{Si}(4)-\mathrm{C}(45)$ & $110.49(8)$ & $\mathrm{C}(8)-\mathrm{C}(9)-\mathrm{C}(1)$ & $119.03(18)$ \\
\hline $\mathrm{C}(43)-\mathrm{Si}(4)-\mathrm{C}(45)$ & $107.20(12)$ & $\mathrm{C}(18)-\mathrm{C}(10)-\mathrm{C}(15)$ & $108.46(15)$ \\
\hline $\mathrm{C}(44)-\mathrm{Si}(4)-\mathrm{C}(45)$ & $107.41(11)$ & $\mathrm{C}(18)-\mathrm{C}(10)-\mathrm{C}(11)$ & $131.25(16)$ \\
\hline $\mathrm{C}(51)-\mathrm{O}(1)-\mathrm{C}(54)$ & $109.65(15)$ & $\mathrm{C}(15)-\mathrm{C}(10)-\mathrm{C}(11)$ & $119.95(15)$ \\
\hline $\mathrm{C}(51)-\mathrm{O}(1)-\mathrm{Zr}(1)$ & $123.86(11)$ & $\mathrm{C}(18)-\mathrm{C}(10)-\mathrm{Zr}(1)$ & $126.42(12)$ \\
\hline $\mathrm{C}(54)-\mathrm{O}(1)-\mathrm{Zr}(1)$ & $125.36(12)$ & $\mathrm{C}(15)-\mathrm{C}(10)-\operatorname{Zr}(1)$ & $72.97(9)$ \\
\hline $\mathrm{C}(9)-\mathrm{C}(1)-\mathrm{C}(5)$ & $119.83(16)$ & $\mathrm{C}(11)-\mathrm{C}(10)-\mathrm{Zr}(1)$ & $67.60(10)$ \\
\hline $\mathrm{C}(9)-\mathrm{C}(1)-\mathrm{C}(2)$ & $130.77(16)$ & $\mathrm{C}(12)-\mathrm{C}(11)-\mathrm{C}(10)$ & $115.69(16)$ \\
\hline$C(5)-C(1)-C(2)$ & $109.35(14)$ & $\mathrm{C}(12)-\mathrm{C}(11)-\mathrm{Zr}(1)$ & $77.53(10)$ \\
\hline$C(9)-C(1)-\operatorname{Zr}(1)$ & $125.74(12)$ & $C(10)-C(11)-\operatorname{Zr}(1)$ & $77.73(10)$ \\
\hline
\end{tabular}




\begin{tabular}{|c|c|}
\hline $\mathrm{C}(13)-\mathrm{C}(12)-\mathrm{C}(11)$ & $122.13(17)$ \\
\hline$C(13)-C(12)-Z r(1)$ & $73.74(10)$ \\
\hline $\mathrm{C}(11)-\mathrm{C}(12)-\mathrm{Zr}(1)$ & $68.28(10)$ \\
\hline$C(12)-C(13)-C(14)$ & $121.86(17)$ \\
\hline $\mathrm{C}(12)-\mathrm{C}(13)-\mathrm{Zr}(1)$ & $73.59(10)$ \\
\hline$C(14)-C(13)-\operatorname{Zr}(1)$ & $68.08(9)$ \\
\hline C(13)-C(14)-C(15) & $115.57(16)$ \\
\hline$C(13)-C(14)-\operatorname{Zr}(1)$ & $77.75(10)$ \\
\hline$C(15)-C(14)-\operatorname{Zr}(1)$ & $77.67(10)$ \\
\hline$C(16)-C(15)-C(10)$ & $108.49(15)$ \\
\hline$C(16)-C(15)-C(14)$ & $130.86(16)$ \\
\hline$C(10)-C(15)-C(14)$ & $120.28(15)$ \\
\hline$C(16)-C(15)-\operatorname{Zr}(1)$ & $126.23(12)$ \\
\hline$C(10)-C(15)-\operatorname{Zr}(1)$ & $73.21(10)$ \\
\hline$C(14)-C(15)-\operatorname{Zr}(1)$ & $67.56(9)$ \\
\hline $\mathrm{C}(17)-\mathrm{C}(16)-\mathrm{C}(15)$ & $104.94(15)$ \\
\hline $\mathrm{C}(17)-\mathrm{C}(16)-\mathrm{Si}(3)$ & $122.41(14)$ \\
\hline $\mathrm{C}(15)-\mathrm{C}(16)-\mathrm{Si}(3)$ & $131.78(14)$ \\
\hline $\mathrm{C}(18)-\mathrm{C}(17)-\mathrm{C}(16)$ & $112.72(17)$ \\
\hline $\mathrm{C}(17)-\mathrm{C}(18)-\mathrm{C}(10)$ & $105.35(15)$ \\
\hline C(17)-C(18)-Si(4) & $122.93(14)$ \\
\hline $\mathrm{C}(10)-\mathrm{C}(18)-\mathrm{Si}(4)$ & $130.70(14)$ \\
\hline $\mathrm{C}(22)-\mathrm{C}(21)-\mathrm{C}(26)$ & $116.65(18)$ \\
\hline $\mathrm{C}(22)-\mathrm{C}(21)-\mathrm{Si}(1)$ & $120.47(14)$ \\
\hline $\mathrm{C}(26)-\mathrm{C}(21)-\mathrm{Si}(1)$ & $122.87(15)$ \\
\hline $\mathrm{C}(23)-\mathrm{C}(22)-\mathrm{C}(21)$ & $121.83(18)$ \\
\hline $\mathrm{C}(22)-\mathrm{C}(23)-\mathrm{C}(24)$ & $120.0(2)$ \\
\hline $\mathrm{C}(25)-\mathrm{C}(24)-\mathrm{C}(23)$ & $119.8(2)$ \\
\hline $\mathrm{C}(24)-\mathrm{C}(25)-\mathrm{C}(26)$ & $119.87(19)$ \\
\hline$C(25)-C(26)-C(21)$ & $121.8(2)$ \\
\hline$C(30)-C(29)-C(34)$ & $117.05(18)$ \\
\hline C(30)-C(29)-Si(2) & $122.07(15)$ \\
\hline $\mathrm{C}(34)-\mathrm{C}(29)-\mathrm{Si}(2)$ & $120.72(15)$ \\
\hline $\mathrm{C}(31)-\mathrm{C}(30)-\mathrm{C}(29)$ & $121.7(2)$ \\
\hline $\mathrm{C}(32)-\mathrm{C}(31)-\mathrm{C}(30)$ & $120.1(2)$ \\
\hline $\mathrm{C}(31)-\mathrm{C}(32)-\mathrm{C}(33)$ & $119.6(2)$ \\
\hline
\end{tabular}

$\begin{array}{ll}\mathrm{C}(32)-\mathrm{C}(33)-\mathrm{C}(34) & 120.2(2) \\ \mathrm{C}(33)-\mathrm{C}(34)-\mathrm{C}(29) & 121.3(2) \\ \mathrm{C}(38)-\mathrm{C}(37)-\mathrm{C}(42) & 116.68(19) \\ \mathrm{C}(38)-\mathrm{C}(37)-\mathrm{Si}(3) & 121.80(16) \\ \mathrm{C}(42)-\mathrm{C}(37)-\mathrm{Si}(3) & 121.52(15) \\ \mathrm{C}(39)-\mathrm{C}(38)-\mathrm{C}(37) & 121.7(2) \\ \mathrm{C}(40)-\mathrm{C}(39)-\mathrm{C}(38) & 120.2(2) \\ \mathrm{C}(39)-\mathrm{C}(40)-\mathrm{C}(41) & 119.8(2) \\ \mathrm{C}(40)-\mathrm{C}(41)-\mathrm{C}(42) & 120.2(3) \\ \mathrm{C}(41)-\mathrm{C}(42)-\mathrm{C}(37) & 121.4(2) \\ \mathrm{C}(50)-\mathrm{C}(45)-\mathrm{C}(46) & 116.4(2) \\ \mathrm{C}(50)-\mathrm{C}(45)-\mathrm{Si}(4) & 121.88(17) \\ \mathrm{C}(46)-\mathrm{C}(45)-\mathrm{Si}(4) & 121.69(17) \\ \mathrm{C}(47)-\mathrm{C}(46)-\mathrm{C}(45) & 121.6(2) \\ \mathrm{C}(48)-\mathrm{C}(47)-\mathrm{C}(46) & 120.6(3) \\ \mathrm{C}(49)-\mathrm{C}(48)-\mathrm{C}(47) & 119.4(3) \\ \mathrm{C}(48)-\mathrm{C}(49)-\mathrm{C}(50) & 120.5(3) \\ \mathrm{C}(49)-\mathrm{C}(50)-\mathrm{C}(45) & 121.5(3) \\ \mathrm{O}(1)-\mathrm{C}(51)-\mathrm{C}(52) & 104.78(19) \\ \mathrm{C}(53)-\mathrm{C}(52)-\mathrm{C}(51) & 105.5(2) \\ \mathrm{C}(52)-\mathrm{C}(53)-\mathrm{C}(54) & 107.1(2) \\ \mathrm{O}(1)-\mathrm{C}(54)-\mathrm{C}(53) & 104.57(19)\end{array}$




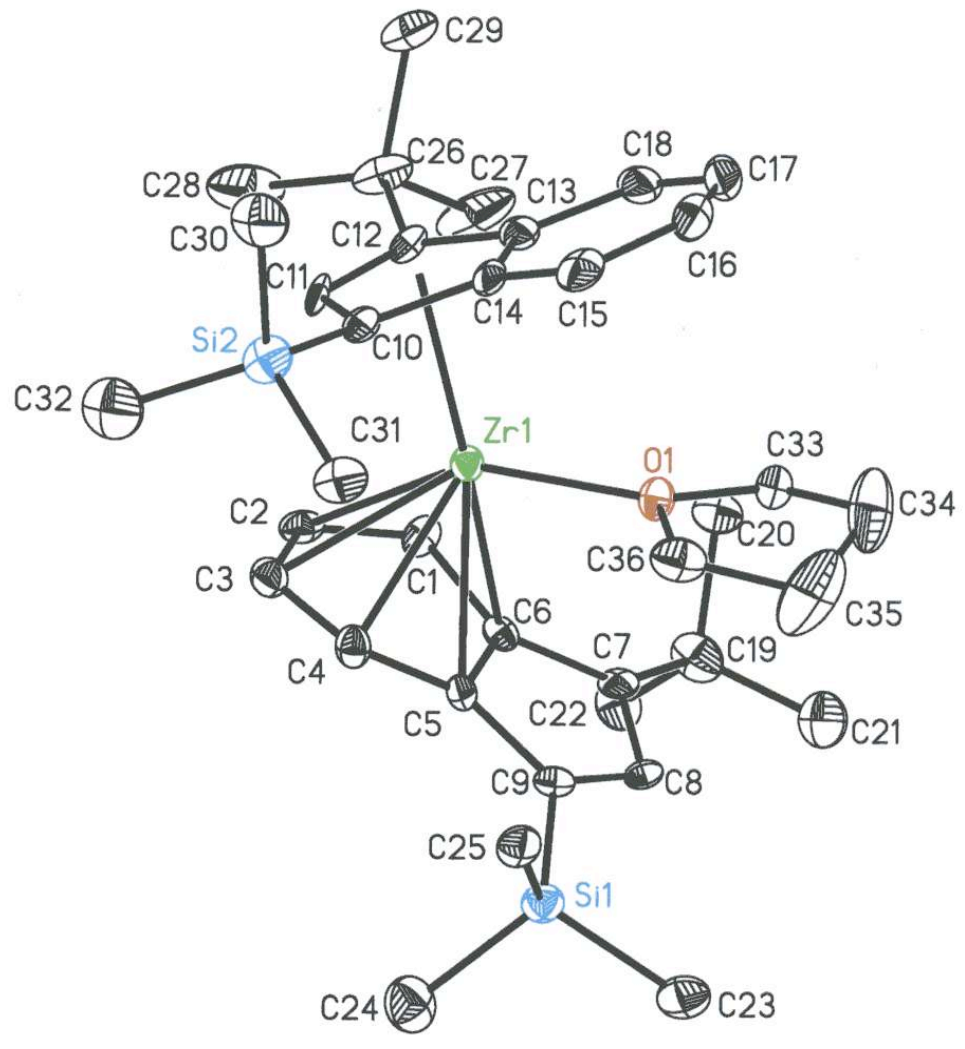

Figure S5. Fully labeled view of the molecular structure of $\mathbf{r a c}-\mathbf{4 - T H F}$ at $30 \%$ probability ellipsoids with hydrogen atoms omitted for clarity. 
Table S4. Crystal data and structure refinement for $\mathbf{r a c - 4 - T H F}$

Identification code

rac-4-THF

Empirical formula

C36 H54 O Si2 Zr

Formula weight

650.19

Temperature

$173(2) \mathrm{K}$

Wavelength

$0.71073 \AA$

Crystal system

Monoclinic

Space group

Unit cell dimensions

P2(1)/c

$\mathrm{a}=11.6523(18) \AA$

$\square=90^{\circ}$.

$\mathrm{b}=19.856(3) \AA$

$\square=102.833(5)^{\circ}$.

$c=15.699(3) \AA$

$\square=90^{\circ}$.

Volume

$3541.6(10) \AA^{3}$

Z

Density (calculated)

4

Absorption coefficient

$1.219 \mathrm{Mg} / \mathrm{m}^{3}$

$0.403 \mathrm{~mm}^{-1}$

$\mathrm{F}(000)$

1384

Crystal size

$0.15 \times 0.10 \times 0.03 \mathrm{~mm}^{3}$

Theta range for data collection

1.68 to $21.96^{\circ}$.

Index ranges

$-12<=\mathrm{h}<=12,-20<=\mathrm{k}<=20,-16<=\mathrm{l}<=16$

Reflections collected

14076

Independent reflections

$4323[\mathrm{R}($ int $)=0.0764]$

Completeness to theta $=21.96^{\circ}$

Absorption correction

$99.7 \%$

Max. and min. transmission

Refinement method

SADABS

Data / restraints / parameters

0.9900 and 0.9420

Full-matrix least-squares on $\mathrm{F}^{2}$

Goodness-of-fit on $\mathrm{F}^{2}$

4323 / 0 / 361

Final $\mathrm{R}$ indices $[\mathrm{I}>2 \operatorname{sigma}(\mathrm{I})]$

$\mathrm{R}$ indices (all data)

1.059

Largest diff. peak and hole

$\mathrm{R} 1=0.0663, \mathrm{wR} 2=0.1576$

$\mathrm{R} 1=0.1018, \mathrm{wR} 2=0.1738$

2.804 and -0.360 e. $\AA^{-3}$ 
Table S5. Bond lengths $[\AA]$ and angles $\left[^{\circ}\right]$ for $\boldsymbol{r a c}-\mathbf{4 - T H F}$.

\begin{tabular}{|c|c|c|c|}
\hline $\mathrm{Zr}(1)-\mathrm{O}(1)$ & $2.259(5)$ & $C(10)-C(14)$ & $1.469(11)$ \\
\hline $\operatorname{Zr}(1)-C(1)$ & $2.328(7)$ & $\mathrm{C}(11)-\mathrm{C}(12)$ & $1.407(10)$ \\
\hline $\mathrm{Zr}(1)-\mathrm{C}(4)$ & $2.358(8)$ & $C(12)-C(13)$ & $1.464(11)$ \\
\hline $\operatorname{Zr}(1)-C(6)$ & $2.421(7)$ & $C(12)-C(26)$ & $1.571(9)$ \\
\hline $\operatorname{Zr}(1)-C(11)$ & $2.466(7)$ & C(13)-C(18) & $1.420(11)$ \\
\hline $\operatorname{Zr}(1)-C(3)$ & $2.472(8)$ & $C(13)-C(14)$ & $1.426(11)$ \\
\hline $\mathrm{Zr}(1)-\mathrm{C}(2)$ & $2.474(8)$ & $C(14)-C(15)$ & $1.438(11)$ \\
\hline $\operatorname{Zr}(1)-C(5)$ & $2.496(7)$ & $C(15)-C(16)$ & $1.371(13)$ \\
\hline $\operatorname{Zr}(1)-C(10)$ & $2.498(7)$ & $C(16)-C(17)$ & $1.360(14)$ \\
\hline $\operatorname{Zr}(1)-C(12)$ & $2.523(7)$ & $\mathrm{C}(17)-\mathrm{C}(18)$ & $1.382(13)$ \\
\hline $\operatorname{Zr}(1)-C(13)$ & $2.591(7)$ & $C(19)-C(21)$ & $1.524(10)$ \\
\hline $\operatorname{Zr}(1)-C(14)$ & $2.610(7)$ & $C(19)-C(22)$ & $1.568(10)$ \\
\hline $\operatorname{Si}(1)-C(9)$ & $1.815(8)$ & $C(19)-C(20)$ & $1.590(10)$ \\
\hline $\mathrm{Si}(1)-\mathrm{C}(23)$ & $1.831(8)$ & $C(26)-C(28)$ & $1.547(12)$ \\
\hline $\operatorname{Si}(1)-C(25)$ & $1.833(8)$ & $C(26)-C(27)$ & $1.590(10)$ \\
\hline $\mathrm{Si}(1)-\mathrm{C}(24)$ & $1.834(9)$ & $C(26)-C(29)$ & $1.609(10)$ \\
\hline $\operatorname{Si}(2)-C(30)$ & $1.806(9)$ & C(33)-C(34) & $1.485(12)$ \\
\hline $\operatorname{Si}(2)-C(32)$ & $1.805(11)$ & $C(34)-C(35)$ & $1.469(16)$ \\
\hline $\operatorname{Si}(2)-C(10)$ & $1.816(8)$ & $C(35)-C(36)$ & $1.482(14)$ \\
\hline $\mathrm{Si}(2)-\mathrm{C}(31)$ & $1.825(9)$ & & \\
\hline $\mathrm{O}(1)-\mathrm{C}(33)$ & $1.454(9)$ & $\mathrm{O}(1)-\mathrm{Zr}(1)-\mathrm{C}(1)$ & $119.6(2)$ \\
\hline $\mathrm{O}(1)-\mathrm{C}(36)$ & $1.463(9)$ & $\mathrm{O}(1)-\mathrm{Zr}(1)-\mathrm{C}(4)$ & $105.0(2)$ \\
\hline$C(1)-C(2)$ & $1.399(11)$ & $C(1)-\operatorname{Zr}(1)-C(4)$ & $76.6(3)$ \\
\hline$C(1)-C(6)$ & $1.479(10)$ & $\mathrm{O}(1)-\mathrm{Zr}(1)-\mathrm{C}(6)$ & $89.2(2)$ \\
\hline$C(2)-C(3)$ & $1.392(11)$ & $C(1)-\operatorname{Zr}(1)-C(6)$ & $36.2(2)$ \\
\hline$C(3)-C(4)$ & $1.395(11)$ & $C(4)-\operatorname{Zr}(1)-C(6)$ & $63.5(2)$ \\
\hline$C(4)-C(5)$ & $1.462(10)$ & $\mathrm{O}(1)-\operatorname{Zr}(1)-\mathrm{C}(11)$ & $131.0(2)$ \\
\hline$C(5)-C(6)$ & $1.441(10)$ & $C(1)-\operatorname{Zr}(1)-C(11)$ & $104.2(2)$ \\
\hline$C(5)-C(9)$ & $1.437(10)$ & $\mathrm{C}(4)-\operatorname{Zr}(1)-\mathrm{C}(11)$ & $105.4(3)$ \\
\hline$C(6)-C(7)$ & $1.427(10)$ & $C(6)-\operatorname{Zr}(1)-C(11)$ & $139.0(2)$ \\
\hline$C(7)-C(8)$ & $1.379(10)$ & $\mathrm{O}(1)-\mathrm{Zr}(1)-\mathrm{C}(3)$ & $138.4(2)$ \\
\hline$C(7)-C(19)$ & $1.600(9)$ & $C(1)-\operatorname{Zr}(1)-C(3)$ & $61.4(3)$ \\
\hline $\mathrm{C}(8)-\mathrm{C}(9)$ & $1.406(10)$ & $C(4)-\operatorname{Zr}(1)-C(3)$ & $33.5(2)$ \\
\hline $\mathrm{C}(10)-\mathrm{C}(11)$ & $1.395(11)$ & $C(6)-\operatorname{Zr}(1)-C(3)$ & $70.3(2)$ \\
\hline
\end{tabular}




\begin{tabular}{|c|c|c|c|}
\hline $\mathrm{C}(11)-\operatorname{Zr}(1)-\mathrm{C}(3)$ & $80.5(3)$ & $C(3)-\operatorname{Zr}(1)-C(13)$ & 133.1(3) \\
\hline $\mathrm{O}(1)-\mathrm{Zr}(1)-\mathrm{C}(2)$ & $148.6(2)$ & $C(2)-\operatorname{Zr}(1)-C(13)$ & $120.1(3)$ \\
\hline$C(1)-\operatorname{Zr}(1)-C(2)$ & $33.7(3)$ & $C(5)-\operatorname{Zr}(1)-C(13)$ & $168.7(2)$ \\
\hline$C(4)-\operatorname{Zr}(1)-C(2)$ & $60.6(3)$ & $C(10)-\operatorname{Zr}(1)-C(13)$ & $55.2(2)$ \\
\hline$C(6)-\operatorname{Zr}(1)-C(2)$ & $59.5(2)$ & $C(12)-\operatorname{Zr}(1)-C(13)$ & $33.2(2)$ \\
\hline $\mathrm{C}(11)-\mathrm{Zr}(1)-\mathrm{C}(2)$ & $80.4(2)$ & $\mathrm{O}(1)-\mathrm{Zr}(1)-\mathrm{C}(14)$ & $78.6(2)$ \\
\hline$C(3)-\operatorname{Zr}(1)-C(2)$ & $32.7(3)$ & $C(1)-\operatorname{Zr}(1)-C(14)$ & $148.5(3)$ \\
\hline $\mathrm{O}(1)-\mathrm{Zr}(1)-\mathrm{C}(5)$ & $84.5(2)$ & $C(4)-\operatorname{Zr}(1)-C(14)$ & $126.2(3)$ \\
\hline$C(1)-\operatorname{Zr}(1)-C(5)$ & $62.9(3)$ & $C(6)-\operatorname{Zr}(1)-C(14)$ & $166.0(2)$ \\
\hline$C(4)-\operatorname{Zr}(1)-C(5)$ & $34.9(2)$ & $C(11)-\operatorname{Zr}(1)-C(14)$ & $52.5(2)$ \\
\hline$C(6)-\operatorname{Zr}(1)-C(5)$ & $34.0(2)$ & $C(3)-\operatorname{Zr}(1)-C(14)$ & $123.6(2)$ \\
\hline$C(11)-\operatorname{Zr}(1)-C(5)$ & $138.2(2)$ & $C(2)-\operatorname{Zr}(1)-C(14)$ & $132.8(2)$ \\
\hline$C(3)-\operatorname{Zr}(1)-C(5)$ & $58.1(2)$ & $C(5)-\operatorname{Zr}(1)-C(14)$ & $148.4(3)$ \\
\hline$C(2)-\operatorname{Zr}(1)-C(5)$ & $68.3(2)$ & $C(10)-\operatorname{Zr}(1)-C(14)$ & $33.3(2)$ \\
\hline $\mathrm{O}(1)-\mathrm{Zr}(1)-\mathrm{C}(10)$ & $104.9(2)$ & $C(12)-\operatorname{Zr}(1)-C(14)$ & $54.2(2)$ \\
\hline$C(1)-\operatorname{Zr}(1)-C(10)$ & $135.0(3)$ & $C(13)-\operatorname{Zr}(1)-C(14)$ & $31.8(2)$ \\
\hline$C(4)-\operatorname{Zr}(1)-C(10)$ & $99.3(3)$ & $C(9)-S i(1)-C(23)$ & $108.3(4)$ \\
\hline$C(6)-\operatorname{Zr}(1)-C(10)$ & $160.5(2)$ & $C(9)-S i(1)-C(25)$ & $113.5(4)$ \\
\hline $\mathrm{C}(11)-\operatorname{Zr}(1)-\mathrm{C}(10)$ & $32.6(2)$ & $C(23)-S i(1)-C(25)$ & $110.6(4)$ \\
\hline$C(3)-\operatorname{Zr}(1)-C(10)$ & $90.3(3)$ & $C(9)-S i(1)-C(24)$ & $110.4(4)$ \\
\hline$C(2)-\operatorname{Zr}(1)-C(10)$ & $105.0(3)$ & $C(23)-\operatorname{Si}(1)-C(24)$ & $107.5(4)$ \\
\hline$C(5)-\operatorname{Zr}(1)-C(10)$ & $132.5(3)$ & $C(25)-S i(1)-C(24)$ & $106.4(4)$ \\
\hline $\mathrm{O}(1)-\mathrm{Zr}(1)-\mathrm{C}(12)$ & $117.8(2)$ & $C(30)-\operatorname{Si}(2)-C(32)$ & $110.9(5)$ \\
\hline$C(1)-\operatorname{Zr}(1)-C(12)$ & $94.5(3)$ & $C(30)-S i(2)-C(10)$ & $106.9(4)$ \\
\hline$C(4)-\operatorname{Zr}(1)-C(12)$ & $134.3(2)$ & $C(32)-\operatorname{Si}(2)-C(10)$ & $109.9(4)$ \\
\hline$C(6)-\operatorname{Zr}(1)-C(12)$ & $128.7(2)$ & $C(30)-\operatorname{Si}(2)-C(31)$ & $108.8(4)$ \\
\hline $\mathrm{C}(11)-\mathrm{Zr}(1)-\mathrm{C}(12)$ & $32.7(2)$ & $C(32)-S i(2)-C(31)$ & $109.8(5)$ \\
\hline $\mathrm{C}(3)-\mathrm{Zr}(1)-\mathrm{C}(12)$ & $103.0(3)$ & $C(10)-S i(2)-C(31)$ & $110.7(4)$ \\
\hline$C(2)-\operatorname{Zr}(1)-C(12)$ & $87.1(2)$ & $\mathrm{C}(33)-\mathrm{O}(1)-\mathrm{C}(36)$ & $108.4(6)$ \\
\hline$C(5)-\operatorname{Zr}(1)-C(12)$ & $155.1(2)$ & $\mathrm{C}(33)-\mathrm{O}(1)-\mathrm{Zr}(1)$ & $126.0(4)$ \\
\hline$C(10)-\operatorname{Zr}(1)-C(12)$ & $56.2(3)$ & $\mathrm{C}(36)-\mathrm{O}(1)-\mathrm{Zr}(1)$ & $124.6(5)$ \\
\hline $\mathrm{O}(1)-\operatorname{Zr}(1)-\mathrm{C}(13)$ & $85.3(2)$ & $C(2)-C(1)-C(6)$ & $115.0(7)$ \\
\hline$C(1)-\operatorname{Zr}(1)-C(13)$ & 119.1(3) & $C(2)-C(1)-\operatorname{Zr}(1)$ & $78.9(4)$ \\
\hline$C(4)-\operatorname{Zr}(1)-C(13)$ & $154.4(3)$ & $C(6)-C(1)-\operatorname{Zr}(1)$ & $75.3(4)$ \\
\hline$C(6)-\operatorname{Zr}(1)-C(13)$ & $141.2(2)$ & $C(3)-C(2)-C(1)$ & $123.1(7)$ \\
\hline$C(11)-\operatorname{Zr}(1)-C(13)$ & $53.1(2)$ & $C(3)-C(2)-Z r(1)$ & $73.6(4)$ \\
\hline
\end{tabular}




\begin{tabular}{|c|c|c|c|}
\hline$C(1)-C(2)-Z r(1)$ & $67.4(4)$ & $\mathrm{C}(11)-\mathrm{C}(12)-\mathrm{C}(26)$ & $126.3(6)$ \\
\hline$C(2)-C(3)-C(4)$ & $122.3(7)$ & $\mathrm{C}(13)-\mathrm{C}(12)-\mathrm{C}(26)$ & $126.6(7)$ \\
\hline$C(2)-C(3)-Z r(1)$ & $73.7(4)$ & $\mathrm{C}(11)-\mathrm{C}(12)-\mathrm{Zr}(1)$ & $71.4(4)$ \\
\hline $\mathrm{C}(4)-\mathrm{C}(3)-\mathrm{Zr}(1)$ & $68.8(4)$ & $\mathrm{C}(13)-\mathrm{C}(12)-\mathrm{Zr}(1)$ & $75.9(4)$ \\
\hline$C(3)-C(4)-C(5)$ & $115.1(7)$ & $C(26)-C(12)-Z r(1)$ & $132.5(4)$ \\
\hline$C(3)-C(4)-Z r(1)$ & $77.8(5)$ & $\mathrm{C}(18)-\mathrm{C}(13)-\mathrm{C}(14)$ & $118.8(8)$ \\
\hline$C(5)-C(4)-Z r(1)$ & $77.7(4)$ & $\mathrm{C}(18)-\mathrm{C}(13)-\mathrm{C}(12)$ & $133.0(8)$ \\
\hline$C(6)-C(5)-C(9)$ & $108.2(6)$ & $\mathrm{C}(14)-\mathrm{C}(13)-\mathrm{C}(12)$ & $108.1(7)$ \\
\hline$C(6)-C(5)-C(4)$ & $120.1(7)$ & $\mathrm{C}(18)-\mathrm{C}(13)-\mathrm{Zr}(1)$ & $122.3(5)$ \\
\hline$C(9)-C(5)-C(4)$ & $131.2(7)$ & $C(14)-C(13)-\operatorname{Zr}(1)$ & $74.8(4)$ \\
\hline$C(6)-C(5)-Z r(1)$ & $70.1(4)$ & $\mathrm{C}(12)-\mathrm{C}(13)-\mathrm{Zr}(1)$ & $70.8(4)$ \\
\hline$C(9)-C(5)-Z r(1)$ & $128.5(5)$ & $\mathrm{C}(13)-\mathrm{C}(14)-\mathrm{C}(15)$ & $119.7(8)$ \\
\hline$C(4)-C(5)-Z r(1)$ & $67.4(4)$ & $C(13)-C(14)-C(10)$ & $109.2(7)$ \\
\hline$C(7)-C(6)-C(5)$ & $107.7(6)$ & $\mathrm{C}(15)-\mathrm{C}(14)-\mathrm{C}(10)$ & $131.2(8)$ \\
\hline$C(7)-C(6)-C(1)$ & $133.0(7)$ & $C(13)-C(14)-Z r(1)$ & $73.4(4)$ \\
\hline$C(5)-C(6)-C(1)$ & $119.3(6)$ & $C(15)-C(14)-\operatorname{Zr}(1)$ & $124.3(5)$ \\
\hline$C(7)-C(6)-Z r(1)$ & $125.9(5)$ & $C(10)-C(14)-Z r(1)$ & $69.2(4)$ \\
\hline$C(5)-C(6)-Z r(1)$ & $75.8(4)$ & $C(16)-C(15)-C(14)$ & $118.0(9)$ \\
\hline$C(1)-C(6)-Z r(1)$ & $68.4(4)$ & $C(17)-C(16)-C(15)$ & $122.8(9)$ \\
\hline$C(8)-C(7)-C(6)$ & $106.2(7)$ & $\mathrm{C}(16)-\mathrm{C}(17)-\mathrm{C}(18)$ & $121.4(9)$ \\
\hline $\mathrm{C}(8)-\mathrm{C}(7)-\mathrm{C}(19)$ & $125.1(6)$ & $\mathrm{C}(17)-\mathrm{C}(18)-\mathrm{C}(13)$ & $119.3(9)$ \\
\hline$C(6)-C(7)-C(19)$ & $128.0(7)$ & $C(21)-C(19)-C(22)$ & $111.3(6)$ \\
\hline $\mathrm{C}(7)-\mathrm{C}(8)-\mathrm{C}(9)$ & $113.0(7)$ & $C(21)-C(19)-C(20)$ & $108.8(6)$ \\
\hline $\mathrm{C}(8)-\mathrm{C}(9)-\mathrm{C}(5)$ & $104.8(7)$ & $C(22)-C(19)-C(20)$ & $107.5(6)$ \\
\hline C(8)-C(9)-Si(1) & $125.7(6)$ & $C(21)-C(19)-C(7)$ & $111.7(6)$ \\
\hline C(5)-C(9)-Si(1) & $127.9(6)$ & $\mathrm{C}(22)-\mathrm{C}(19)-\mathrm{C}(7)$ & $107.2(5)$ \\
\hline$C(11)-C(10)-C(14)$ & $103.3(7)$ & $C(20)-C(19)-C(7)$ & $110.3(5)$ \\
\hline $\mathrm{C}(11)-\mathrm{C}(10)-\mathrm{Si}(2)$ & $127.0(6)$ & $C(28)-C(26)-C(12)$ & $112.8(6)$ \\
\hline $\mathrm{C}(14)-\mathrm{C}(10)-\mathrm{Si}(2)$ & $126.2(6)$ & $C(28)-C(26)-C(27)$ & $110.3(7)$ \\
\hline$C(11)-C(10)-\operatorname{Zr}(1)$ & $72.4(4)$ & $C(12)-C(26)-C(27)$ & $112.6(6)$ \\
\hline$C(14)-C(10)-Z r(1)$ & $77.5(4)$ & $C(28)-C(26)-C(29)$ & $108.4(6)$ \\
\hline $\mathrm{Si}(2)-\mathrm{C}(10)-\mathrm{Zr}(1)$ & $130.6(4)$ & $C(12)-C(26)-C(29)$ & $106.4(5)$ \\
\hline$C(10)-C(11)-C(12)$ & $115.1(7)$ & $C(27)-C(26)-C(29)$ & $105.9(6)$ \\
\hline$C(10)-C(11)-\operatorname{Zr}(1)$ & $75.0(4)$ & $\mathrm{O}(1)-\mathrm{C}(33)-\mathrm{C}(34)$ & $106.0(7)$ \\
\hline$C(12)-C(11)-\operatorname{Zr}(1)$ & $75.9(4)$ & $C(35)-C(34)-C(33)$ & $104.3(9)$ \\
\hline $\mathrm{C}(11)-\mathrm{C}(12)-\mathrm{C}(13)$ & $103.9(6)$ & $\mathrm{C}(34)-\mathrm{C}(35)-\mathrm{C}(36)$ & $105.8(9)$ \\
\hline
\end{tabular}


$\mathrm{O}(1)-\mathrm{C}(36)-\mathrm{C}(35)$

106.0(8) 


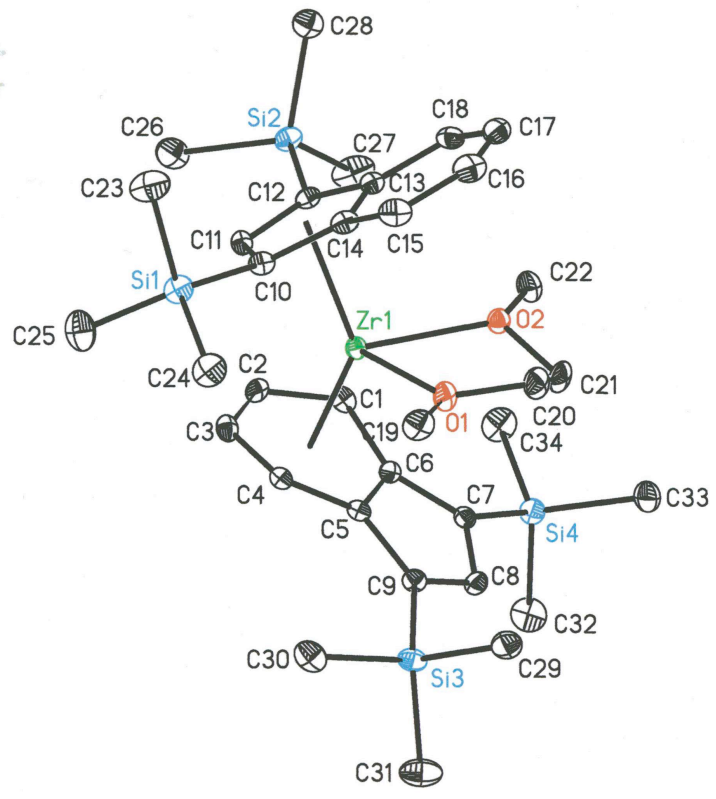

Figure S6a. Fully labeled view of one molecule of 1-DME at $30 \%$ probability ellipsoids. Hydrogen atoms omitted for clarity.
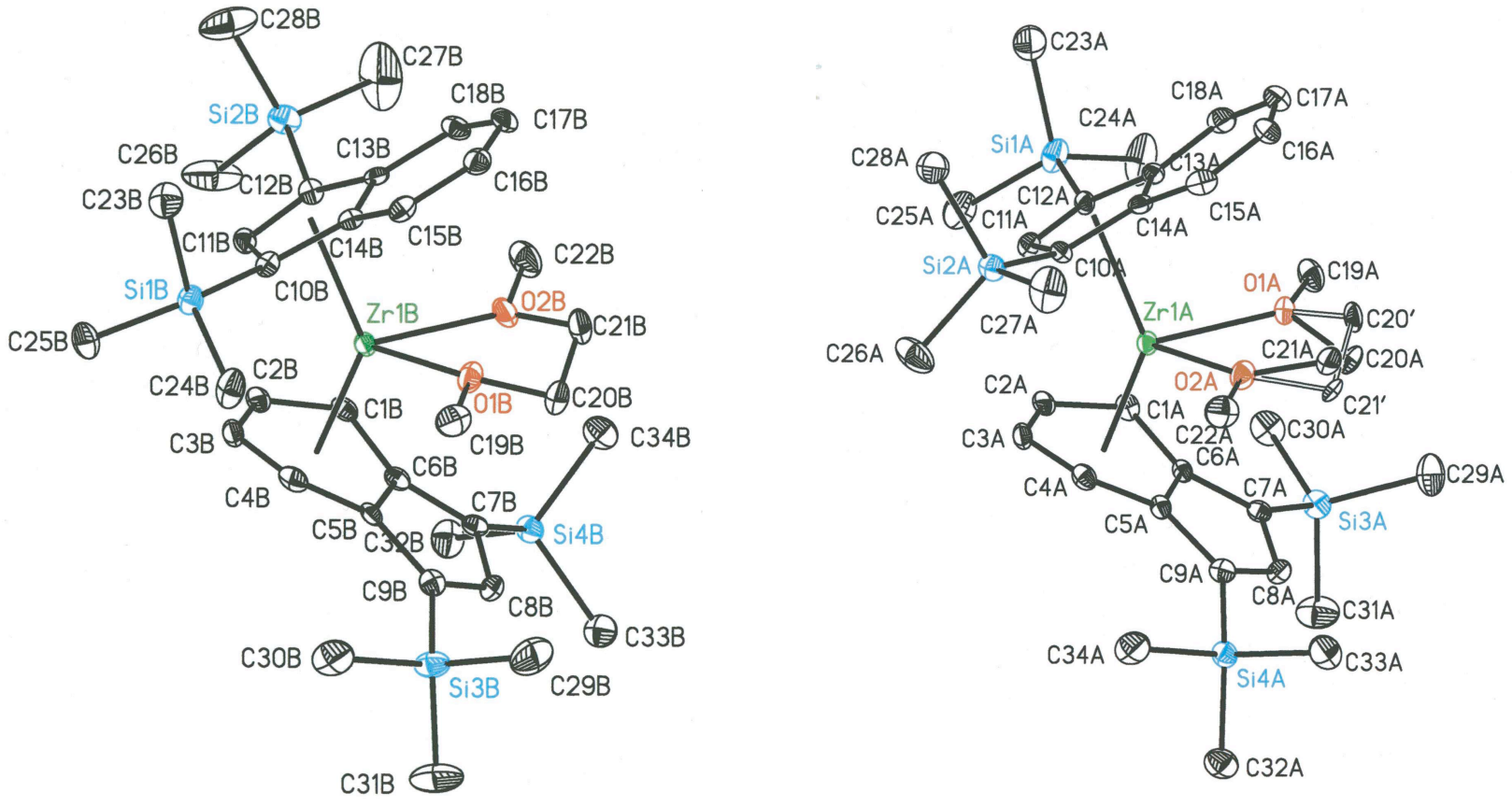

Figure S6b. Fully labeled views of the second (left) and third (right) molecules of 1-DME at $30 \%$ probability ellipsoids. Hydrogen atoms omitted for clarity. 
Table S6. Crystal data and structure refinement for 1-DME.

Identification code

Empirical formula

Formula weight

Temperature

Wavelength

Crystal system

Space group

Unit cell dimensions

Volume

Z

Density (calculated)

Absorption coefficient

$\mathrm{F}(000)$

Crystal size

Theta range for data collection

Index ranges

Reflections collected

Independent reflections

Completeness to theta $=23.82^{\circ}$

Absorption correction

Max. and min. transmission

Refinement method

Data / restraints / parameters

Goodness-of-fit on $\mathrm{F}^{2}$

Final $R$ indices $[\mathrm{I}>2 \operatorname{sigma}(\mathrm{I})]$

$\mathrm{R}$ indices (all data)

Largest diff. peak and hole $\operatorname{cb} 55$

C102 H168 O6 Si12 Zr3

2101.10

173(2) K

$0.71073 \AA$

Monoclinic

$\mathrm{P} 2(1) / \mathrm{c}$

$\mathrm{a}=20.7926(17) \AA \quad \square=90^{\circ}$.

$\mathrm{b}=25.896(2) \AA$

$\square=106.506(3)^{\circ}$.

$\mathrm{c}=21.9514(12) \AA$

$\square=90^{\circ}$.

$11332.5(14) \AA^{3}$

4

$1.231 \mathrm{Mg} / \mathrm{m}^{3}$

$0.445 \mathrm{~mm}^{-1}$

4464

$0.50 \times 0.10 \times 0.05 \mathrm{~mm}^{3}$

1.25 to $23.82^{\circ}$.

$-23<=\mathrm{h}<=22,-29<=\mathrm{k}<=29,-24<=\mathrm{l}<=14$

40487

$13931[\mathrm{R}($ int $)=0.0553]$

$79.9 \%$

Semiempirical by SADABS

0.9781 and 0.8082

Full-matrix least-squares on $\mathrm{F}^{2}$

$13931 / 0 / 1321$

0.961

$\mathrm{R} 1=0.0363, \mathrm{wR} 2=0.0714$

$\mathrm{R} 1=0.0728, \mathrm{wR} 2=0.0812$ 
Table S7. Bond lengths $[\AA]$ and angles $\left[{ }^{\circ}\right]$ for 1-DME.

\begin{tabular}{|c|c|c|c|}
\hline $\mathrm{Zr}(1)-\mathrm{O}(2)$ & $2.305(2)$ & $\mathrm{C}(2)-\mathrm{C}(3)$ & $1.394(5)$ \\
\hline $\mathrm{Zr}(1)-\mathrm{O}(1)$ & $2.326(3)$ & $C(3)-C(4)$ & $1.412(5)$ \\
\hline $\operatorname{Zr}(1)-C(4)$ & $2.340(4)$ & $C(4)-C(5)$ & $1.455(5)$ \\
\hline $\operatorname{Zr}(1)-C(1)$ & $2.368(4)$ & $\mathrm{C}(5)-\mathrm{C}(9)$ & $1.428(5)$ \\
\hline $\operatorname{Zr}(1)-C(11)$ & $2.433(4)$ & $C(5)-C(6)$ & $1.432(5)$ \\
\hline $\operatorname{Zr}(1)-C(3)$ & $2.448(4)$ & $C(6)-C(7)$ & $1.433(4)$ \\
\hline $\mathrm{Zr}(1)-\mathrm{C}(2)$ & $2.469(4)$ & $C(7)-C(8)$ & $1.414(5)$ \\
\hline $\operatorname{Zr}(1)-C(10)$ & $2.508(3)$ & $\mathrm{C}(8)-\mathrm{C}(9)$ & $1.417(5)$ \\
\hline $\operatorname{Zr}(1)-C(12)$ & $2.516(3)$ & $\mathrm{C}(10)-\mathrm{C}(11)$ & $1.418(5)$ \\
\hline $\operatorname{Zr}(1)-C(5)$ & $2.556(3)$ & $C(10)-C(14)$ & $1.459(5)$ \\
\hline $\operatorname{Zr}(1)-C(6)$ & $2.584(3)$ & $C(11)-C(12)$ & $1.428(5)$ \\
\hline $\operatorname{Zr}(1)-C(14)$ & $2.643(3)$ & $\mathrm{C}(12)-\mathrm{C}(13)$ & $1.451(5)$ \\
\hline $\operatorname{Si}(1)-C(10)$ & $1.855(4)$ & $C(13)-C(18)$ & $1.415(5)$ \\
\hline $\operatorname{Si}(1)-C(25)$ & $1.860(4)$ & $C(13)-C(14)$ & $1.436(5)$ \\
\hline $\mathrm{Si}(1)-\mathrm{C}(24)$ & $1.864(4)$ & $C(14)-C(15)$ & $1.397(6)$ \\
\hline $\operatorname{Si}(1)-C(23)$ & $1.870(4)$ & $C(15)-C(16)$ & $1.353(6)$ \\
\hline $\mathrm{Si}(2)-\mathrm{C}(12)$ & $1.858(4)$ & $C(16)-C(17)$ & $1.415(6)$ \\
\hline $\operatorname{Si}(2)-C(27)$ & $1.860(4)$ & $\mathrm{C}(17)-\mathrm{C}(18)$ & $1.358(6)$ \\
\hline $\operatorname{Si}(2)-C(28)$ & $1.862(4)$ & $C(20)-C(21)$ & $1.472(6)$ \\
\hline $\mathrm{Si}(2)-\mathrm{C}(26)$ & $1.873(4)$ & $\operatorname{Zr}(1 \mathrm{~A})-\mathrm{O}(1 \mathrm{~A})$ & $2.296(3)$ \\
\hline $\operatorname{Si}(3)-C(9)$ & $1.840(4)$ & $\mathrm{Zr}(1 \mathrm{~A})-\mathrm{O}(2 \mathrm{~A})$ & $2.311(3)$ \\
\hline $\mathrm{Si}(3)-\mathrm{C}(29)$ & $1.871(4)$ & $\operatorname{Zr}(1 \mathrm{~A})-\mathrm{C}(1 \mathrm{~A})$ & $2.355(4)$ \\
\hline $\mathrm{Si}(3)-\mathrm{C}(30)$ & $1.875(4)$ & $\operatorname{Zr}(1 \mathrm{~A})-\mathrm{C}(4 \mathrm{~A})$ & $2.367(4)$ \\
\hline $\operatorname{Si}(3)-C(31)$ & $1.876(4)$ & $\operatorname{Zr}(1 \mathrm{~A})-\mathrm{C}(11 \mathrm{~A})$ & $2.425(4)$ \\
\hline $\operatorname{Si}(4)-C(7)$ & $1.834(4)$ & $\operatorname{Zr}(1 \mathrm{~A})-\mathrm{C}(2 \mathrm{~A})$ & $2.461(4)$ \\
\hline $\operatorname{Si}(4)-C(32)$ & $1.865(4)$ & $\operatorname{Zr}(1 \mathrm{~A})-\mathrm{C}(3 \mathrm{~A})$ & $2.467(4)$ \\
\hline $\mathrm{Si}(4)-\mathrm{C}(33)$ & $1.867(4)$ & $\operatorname{Zr}(1 \mathrm{~A})-\mathrm{C}(10 \mathrm{~A})$ & $2.479(3)$ \\
\hline $\operatorname{Si}(4)-C(34)$ & $1.875(4)$ & $\operatorname{Zr}(1 \mathrm{~A})-\mathrm{C}(12 \mathrm{~A})$ & $2.530(3)$ \\
\hline $\mathrm{O}(1)-\mathrm{C}(19)$ & $1.436(4)$ & $\operatorname{Zr}(1 \mathrm{~A})-\mathrm{C}(6 \mathrm{~A})$ & $2.555(3)$ \\
\hline $\mathrm{O}(1)-\mathrm{C}(20)$ & $1.439(5)$ & $\operatorname{Zr}(1 \mathrm{~A})-\mathrm{C}(5 \mathrm{~A})$ & $2.583(3)$ \\
\hline $\mathrm{O}(2)-\mathrm{C}(22)$ & $1.431(4)$ & $\operatorname{Zr}(1 \mathrm{~A})-\mathrm{C}(14 \mathrm{~A})$ & $2.628(3)$ \\
\hline $\mathrm{O}(2)-\mathrm{C}(21)$ & $1.444(4)$ & $\mathrm{Si}(1 \mathrm{~A})-\mathrm{C}(12 \mathrm{~A})$ & $1.859(4)$ \\
\hline $\mathrm{C}(1)-\mathrm{C}(2)$ & $1.412(5)$ & $\operatorname{Si}(1 \mathrm{~A})-\mathrm{C}(23 \mathrm{~A})$ & $1.865(4)$ \\
\hline$C(1)-C(6)$ & $1.457(5)$ & $\mathrm{Si}(1 \mathrm{~A})-\mathrm{C}(25 \mathrm{~A})$ & $1.866(4)$ \\
\hline
\end{tabular}




\begin{tabular}{|c|c|c|c|}
\hline $\operatorname{Si}(1 \mathrm{~A})-\mathrm{C}(24 \mathrm{~A})$ & $1.877(4)$ & $C(15 A)-C(16 A)$ & $1.353(6)$ \\
\hline $\operatorname{Si}(2 A)-C(10 A)$ & $1.850(4)$ & $C(16 A)-C(17 A)$ & $1.397(6)$ \\
\hline $\operatorname{Si}(2 A)-C(28 A)$ & $1.855(4)$ & $\mathrm{C}(17 \mathrm{~A})-\mathrm{C}(18 \mathrm{~A})$ & $1.360(6)$ \\
\hline $\operatorname{Si}(2 A)-C(26 A)$ & $1.858(4)$ & $C(20 A)-C(21 A)$ & $1.43(3)$ \\
\hline $\operatorname{Si}(2 A)-C(27 A)$ & $1.869(4)$ & $\mathrm{C}\left(20^{\prime}\right)-\mathrm{C}\left(21^{\prime}\right)$ & $1.56(4)$ \\
\hline $\operatorname{Si}(3 \mathrm{~A})-\mathrm{C}(7 \mathrm{~A})$ & $1.841(4)$ & $\mathrm{Zr}(1 \mathrm{~B})-\mathrm{O}(1 \mathrm{~B})$ & $2.297(3)$ \\
\hline $\operatorname{Si}(3 \mathrm{~A})-\mathrm{C}(30 \mathrm{~A})$ & $1.865(4)$ & $\mathrm{Zr}(1 \mathrm{~B})-\mathrm{O}(2 \mathrm{~B})$ & $2.312(3)$ \\
\hline $\operatorname{Si}(3 A)-C(29 A)$ & $1.865(4)$ & $\mathrm{Zr}(1 \mathrm{~B})-\mathrm{C}(4 \mathrm{~B})$ & $2.341(4)$ \\
\hline $\operatorname{Si}(3 \mathrm{~A})-\mathrm{C}(31 \mathrm{~A})$ & $1.871(4)$ & $\mathrm{Zr}(1 \mathrm{~B})-\mathrm{C}(1 \mathrm{~B})$ & $2.380(4)$ \\
\hline $\mathrm{Si}(4 \mathrm{~A})-\mathrm{C}(9 \mathrm{~A})$ & $1.846(4)$ & $\mathrm{Zr}(1 \mathrm{~B})-\mathrm{C}(11 \mathrm{~B})$ & $2.425(4)$ \\
\hline $\operatorname{Si}(4 A)-C(33 A)$ & $1.868(4)$ & $\mathrm{Zr}(1 \mathrm{~B})-\mathrm{C}(3 \mathrm{~B})$ & $2.448(4)$ \\
\hline $\operatorname{Si}(4 A)-C(32 A)$ & $1.870(4)$ & $\mathrm{Zr}(1 \mathrm{~B})-\mathrm{C}(2 \mathrm{~B})$ & $2.474(4)$ \\
\hline $\operatorname{Si}(4 \mathrm{~A})-\mathrm{C}(34 \mathrm{~A})$ & $1.876(4)$ & $\mathrm{Zr}(1 \mathrm{~B})-\mathrm{C}(12 \mathrm{~B})$ & 2.493(3) \\
\hline $\mathrm{O}(1 \mathrm{~A})-\mathrm{C}\left(20^{\prime}\right)$ & $1.347(18)$ & $\mathrm{Zr}(1 \mathrm{~B})-\mathrm{C}(10 \mathrm{~B})$ & $2.516(3)$ \\
\hline $\mathrm{O}(1 \mathrm{~A})-\mathrm{C}(19 \mathrm{~A})$ & $1.434(4)$ & $\mathrm{Zr}(1 \mathrm{~B})-\mathrm{C}(5 \mathrm{~B})$ & $2.575(3)$ \\
\hline $\mathrm{O}(1 \mathrm{~A})-\mathrm{C}(20 \mathrm{~A})$ & $1.514(15)$ & $\mathrm{Zr}(1 \mathrm{~B})-\mathrm{C}(6 \mathrm{~B})$ & $2.580(3)$ \\
\hline $\mathrm{O}(2 \mathrm{~A})-\mathrm{C}(21 \mathrm{~A})$ & $1.37(2)$ & $\mathrm{Zr}(1 \mathrm{~B})-\mathrm{C}(13 \mathrm{~B})$ & $2.643(3)$ \\
\hline $\mathrm{O}(2 \mathrm{~A})-\mathrm{C}(22 \mathrm{~A})$ & $1.421(4)$ & $\mathrm{Si}(1 \mathrm{~B})-\mathrm{C}(25 \mathrm{~B})$ & $1.857(4)$ \\
\hline $\mathrm{O}(2 \mathrm{~A})-\mathrm{C}\left(21^{\prime}\right)$ & $1.50(2)$ & $\mathrm{Si}(1 \mathrm{~B})-\mathrm{C}(10 \mathrm{~B})$ & $1.863(4)$ \\
\hline$C(1 \mathrm{~A})-\mathrm{C}(2 \mathrm{~A})$ & $1.414(5)$ & $\mathrm{Si}(1 \mathrm{~B})-\mathrm{C}(23 \mathrm{~B})$ & $1.864(4)$ \\
\hline$C(1 \mathrm{~A})-\mathrm{C}(6 \mathrm{~A})$ & $1.456(5)$ & $\mathrm{Si}(1 \mathrm{~B})-\mathrm{C}(24 \mathrm{~B})$ & $1.870(4)$ \\
\hline$C(2 A)-C(3 A)$ & $1.394(6)$ & $\mathrm{Si}(2 \mathrm{~B})-\mathrm{C}(26 \mathrm{~B})$ & $1.832(4)$ \\
\hline$C(3 A)-C(4 A)$ & $1.409(5)$ & $\mathrm{Si}(2 \mathrm{~B})-\mathrm{C}(28 \mathrm{~B})$ & $1.845(4)$ \\
\hline$C(4 A)-C(5 A)$ & $1.459(5)$ & $\mathrm{Si}(2 \mathrm{~B})-\mathrm{C}(12 \mathrm{~B})$ & $1.853(4)$ \\
\hline$C(5 A)-C(9 A)$ & $1.429(5)$ & $\mathrm{Si}(2 \mathrm{~B})-\mathrm{C}(27 \mathrm{~B})$ & $1.859(5)$ \\
\hline$C(5 A)-C(6 A)$ & $1.437(5)$ & $\mathrm{Si}(3 \mathrm{~B})-\mathrm{C}(9 \mathrm{~B})$ & $1.840(4)$ \\
\hline$C(6 A)-C(7 A)$ & $1.438(5)$ & $\mathrm{Si}(3 \mathrm{~B})-\mathrm{C}(31 \mathrm{~B})$ & $1.857(4)$ \\
\hline$C(7 A)-C(8 A)$ & $1.407(5)$ & $\mathrm{Si}(3 \mathrm{~B})-\mathrm{C}(30 \mathrm{~B})$ & $1.858(4)$ \\
\hline $\mathrm{C}(8 \mathrm{~A})-\mathrm{C}(9 \mathrm{~A})$ & $1.412(5)$ & $\mathrm{Si}(3 \mathrm{~B})-\mathrm{C}(29 \mathrm{~B})$ & $1.873(5)$ \\
\hline$C(10 A)-C(11 A)$ & $1.433(5)$ & $\mathrm{Si}(4 \mathrm{~B})-\mathrm{C}(7 \mathrm{~B})$ & $1.845(4)$ \\
\hline$C(10 A)-C(14 A)$ & $1.460(5)$ & $\mathrm{Si}(4 \mathrm{~B})-\mathrm{C}(34 \mathrm{~B})$ & $1.864(4)$ \\
\hline$C(11 \mathrm{~A})-\mathrm{C}(12 \mathrm{~A})$ & $1.416(5)$ & $\mathrm{Si}(4 \mathrm{~B})-\mathrm{C}(33 \mathrm{~B})$ & $1.865(4)$ \\
\hline $\mathrm{C}(12 \mathrm{~A})-\mathrm{C}(13 \mathrm{~A})$ & $1.447(5)$ & $\mathrm{Si}(4 \mathrm{~B})-\mathrm{C}(32 \mathrm{~B})$ & $1.869(4)$ \\
\hline$C(13 \mathrm{~A})-\mathrm{C}(18 \mathrm{~A})$ & $1.393(6)$ & $\mathrm{O}(1 \mathrm{~B})-\mathrm{C}(19 \mathrm{~B})$ & $1.420(4)$ \\
\hline$C(13 A)-C(14 A)$ & $1.427(5)$ & $\mathrm{O}(1 \mathrm{~B})-\mathrm{C}(20 \mathrm{~B})$ & $1.454(5)$ \\
\hline$C(14 \mathrm{~A})-\mathrm{C}(15 \mathrm{~A})$ & $1.421(6)$ & $\mathrm{O}(2 \mathrm{~B})-\mathrm{C}(22 \mathrm{~B})$ & $1.429(5)$ \\
\hline
\end{tabular}




\begin{tabular}{|c|c|c|c|}
\hline $\mathrm{O}(2 \mathrm{~B})-\mathrm{C}(21 \mathrm{~B})$ & $1.438(5)$ & $C(1)-\operatorname{Zr}(1)-C(3)$ & $61.00(14)$ \\
\hline $\mathrm{C}(1 \mathrm{~B})-\mathrm{C}(2 \mathrm{~B})$ & $1.397(5)$ & $C(11)-\operatorname{Zr}(1)-C(3)$ & $76.37(13)$ \\
\hline $\mathrm{C}(1 \mathrm{~B})-\mathrm{C}(6 \mathrm{~B})$ & $1.463(5)$ & $\mathrm{O}(2)-\mathrm{Zr}(1)-\mathrm{C}(2)$ & $121.12(12)$ \\
\hline$C(2 B)-C(3 B)$ & $1.393(6)$ & $\mathrm{O}(1)-\mathrm{Zr}(1)-\mathrm{C}(2)$ & $145.90(11)$ \\
\hline $\mathrm{C}(3 \mathrm{~B})-\mathrm{C}(4 \mathrm{~B})$ & $1.416(6)$ & $C(4)-\operatorname{Zr}(1)-C(2)$ & $61.25(15)$ \\
\hline $\mathrm{C}(4 \mathrm{~B})-\mathrm{C}(5 \mathrm{~B})$ & $1.447(5)$ & $C(1)-\operatorname{Zr}(1)-C(2)$ & $33.86(13)$ \\
\hline $\mathrm{C}(5 \mathrm{~B})-\mathrm{C}(6 \mathrm{~B})$ & $1.432(5)$ & $C(11)-\operatorname{Zr}(1)-C(2)$ & $77.32(14)$ \\
\hline $\mathrm{C}(5 \mathrm{~B})-\mathrm{C}(9 \mathrm{~B})$ & $1.436(5)$ & $C(3)-\operatorname{Zr}(1)-C(2)$ & $32.93(13)$ \\
\hline $\mathrm{C}(6 \mathrm{~B})-\mathrm{C}(7 \mathrm{~B})$ & $1.442(5)$ & $\mathrm{O}(2)-\operatorname{Zr}(1)-\mathrm{C}(10)$ & $125.51(10)$ \\
\hline $\mathrm{C}(7 \mathrm{~B})-\mathrm{C}(8 \mathrm{~B})$ & $1.403(5)$ & $\mathrm{O}(1)-\operatorname{Zr}(1)-\mathrm{C}(10)$ & $93.93(11)$ \\
\hline $\mathrm{C}(8 \mathrm{~B})-\mathrm{C}(9 \mathrm{~B})$ & $1.413(5)$ & $C(4)-\operatorname{Zr}(1)-C(10)$ & $96.50(13)$ \\
\hline$C(10 B)-C(11 B)$ & $1.423(5)$ & $C(1)-\operatorname{Zr}(1)-C(10)$ & $134.78(13)$ \\
\hline$C(10 B)-C(14 B)$ & $1.454(5)$ & $C(11)-\operatorname{Zr}(1)-C(10)$ & $33.31(12)$ \\
\hline$C(11 B)-C(12 B)$ & $1.425(5)$ & $C(3)-\operatorname{Zr}(1)-C(10)$ & $87.03(12)$ \\
\hline$C(12 B)-C(13 B)$ & $1.445(5)$ & $C(2)-\operatorname{Zr}(1)-C(10)$ & $102.98(13)$ \\
\hline$C(13 B)-C(18 B)$ & $1.417(5)$ & $\mathrm{O}(2)-\operatorname{Zr}(1)-\mathrm{C}(12)$ & $94.43(10)$ \\
\hline$C(13 B)-C(14 B)$ & $1.434(5)$ & $\mathrm{O}(1)-\operatorname{Zr}(1)-\mathrm{C}(12)$ & $128.54(11)$ \\
\hline$C(14 B)-C(15 B)$ & $1.396(5)$ & $C(4)-\operatorname{Zr}(1)-C(12)$ & $132.29(13)$ \\
\hline$C(15 B)-C(16 B)$ & $1.349(6)$ & $C(1)-\operatorname{Zr}(1)-C(12)$ & $95.91(13)$ \\
\hline$C(16 B)-C(17 B)$ & $1.397(6)$ & $\mathrm{C}(11)-\operatorname{Zr}(1)-\mathrm{C}(12)$ & $33.49(12)$ \\
\hline $\mathrm{C}(17 \mathrm{~B})-\mathrm{C}(18 \mathrm{~B})$ & $1.364(6)$ & $C(3)-\operatorname{Zr}(1)-C(12)$ & $100.30(13)$ \\
\hline \multirow[t]{2}{*}{$\mathrm{C}(20 \mathrm{~B})-\mathrm{C}(21 \mathrm{~B})$} & $1.479(7)$ & $C(2)-\operatorname{Zr}(1)-C(12)$ & $85.18(13)$ \\
\hline & & $C(10)-\operatorname{Zr}(1)-C(12)$ & $56.88(12)$ \\
\hline $\mathrm{O}(2)-\mathrm{Zr}(1)-\mathrm{O}(1)$ & $67.57(9)$ & $\mathrm{O}(2)-\mathrm{Zr}(1)-\mathrm{C}(5)$ & $97.74(10)$ \\
\hline $\mathrm{O}(2)-\mathrm{Zr}(1)-\mathrm{C}(4)$ & $131.09(11)$ & $\mathrm{O}(1)-\mathrm{Zr}(1)-\mathrm{C}(5)$ & $78.48(11)$ \\
\hline $\mathrm{O}(1)-\mathrm{Zr}(1)-\mathrm{C}(4)$ & $87.82(12)$ & $C(4)-\operatorname{Zr}(1)-C(5)$ & $34.22(11)$ \\
\hline $\mathrm{O}(2)-\mathrm{Zr}(1)-\mathrm{C}(1)$ & $88.30(12)$ & $C(1)-\operatorname{Zr}(1)-C(5)$ & $60.60(13)$ \\
\hline $\mathrm{O}(1)-\mathrm{Zr}(1)-\mathrm{C}(1)$ & $129.19(11)$ & $C(11)-\operatorname{Zr}(1)-C(5)$ & $133.85(13)$ \\
\hline$C(4)-\operatorname{Zr}(1)-C(1)$ & $75.20(15)$ & $C(3)-\operatorname{Zr}(1)-C(5)$ & $57.88(12)$ \\
\hline $\mathrm{O}(2)-\mathrm{Zr}(1)-\mathrm{C}(11)$ & $126.55(11)$ & $C(2)-\operatorname{Zr}(1)-C(5)$ & $67.87(13)$ \\
\hline $\mathrm{O}(1)-\operatorname{Zr}(1)-\mathrm{C}(11)$ & $126.56(12)$ & $C(10)-\operatorname{Zr}(1)-C(5)$ & $129.52(12)$ \\
\hline$C(4)-\operatorname{Zr}(1)-C(11)$ & $102.21(13)$ & $C(12)-\operatorname{Zr}(1)-C(5)$ & $152.97(13)$ \\
\hline$C(1)-\operatorname{Zr}(1)-C(11)$ & $103.93(14)$ & $\mathrm{O}(2)-\mathrm{Zr}(1)-\mathrm{C}(6)$ & $79.79(10)$ \\
\hline $\mathrm{O}(2)-\mathrm{Zr}(1)-\mathrm{C}(3)$ & $146.94(11)$ & $\mathrm{O}(1)-\mathrm{Zr}(1)-\mathrm{C}(6)$ & $96.19(10)$ \\
\hline $\mathrm{O}(1)-\mathrm{Zr}(1)-\mathrm{C}(3)$ & $121.27(12)$ & $C(4)-\operatorname{Zr}(1)-C(6)$ & $60.89(13)$ \\
\hline$C(4)-\operatorname{Zr}(1)-C(3)$ & $34.20(13)$ & $\mathrm{C}(1)-\operatorname{Zr}(1)-\mathrm{C}(6)$ & $33.87(12)$ \\
\hline
\end{tabular}




\begin{tabular}{|c|c|c|c|}
\hline$C(11)-\operatorname{Zr}(1)-C(6)$ & $134.70(13)$ & $C(7)-S i(4)-C(33)$ & $110.62(19)$ \\
\hline$C(3)-\operatorname{Zr}(1)-C(6)$ & $67.90(12)$ & $C(32)-\operatorname{Si}(4)-C(33)$ & $105.25(18)$ \\
\hline$C(2)-\operatorname{Zr}(1)-C(6)$ & $57.52(12)$ & $C(7)-S i(4)-C(34)$ & $113.73(17)$ \\
\hline$C(10)-\operatorname{Zr}(1)-C(6)$ & $154.70(12)$ & $\mathrm{C}(32)-\mathrm{Si}(4)-\mathrm{C}(34)$ & $105.89(19)$ \\
\hline $\mathrm{C}(12)-\mathrm{Zr}(1)-\mathrm{C}(6)$ & $128.93(12)$ & $\mathrm{C}(33)-\mathrm{Si}(4)-\mathrm{C}(34)$ & 109.29(19) \\
\hline$C(5)-\operatorname{Zr}(1)-C(6)$ & $32.34(11)$ & $\mathrm{C}(19)-\mathrm{O}(1)-\mathrm{C}(20)$ & $112.0(3)$ \\
\hline $\mathrm{O}(2)-\mathrm{Zr}(1)-\mathrm{C}(14)$ & $92.80(11)$ & $\mathrm{C}(19)-\mathrm{O}(1)-\mathrm{Zr}(1)$ & $126.3(2)$ \\
\hline $\mathrm{O}(1)-\operatorname{Zr}(1)-\mathrm{C}(14)$ & $77.98(11)$ & $\mathrm{C}(20)-\mathrm{O}(1)-\mathrm{Zr}(1)$ & $120.6(3)$ \\
\hline$C(4)-\operatorname{Zr}(1)-C(14)$ & $123.73(14)$ & $\mathrm{C}(22)-\mathrm{O}(2)-\mathrm{C}(21)$ & $113.0(3)$ \\
\hline$C(1)-\operatorname{Zr}(1)-C(14)$ & $150.11(14)$ & $\mathrm{C}(22)-\mathrm{O}(2)-\mathrm{Zr}(1)$ & $123.5(2)$ \\
\hline $\mathrm{C}(11)-\operatorname{Zr}(1)-\mathrm{C}(14)$ & $52.66(13)$ & $\mathrm{C}(21)-\mathrm{O}(2)-\mathrm{Zr}(1)$ & $116.5(2)$ \\
\hline$C(3)-\operatorname{Zr}(1)-C(14)$ & 119.81(13) & $C(2)-C(1)-C(6)$ & $116.0(4)$ \\
\hline $\mathrm{C}(2)-\mathrm{Zr}(1)-\mathrm{C}(14)$ & $129.98(12)$ & $\mathrm{C}(2)-\mathrm{C}(1)-\operatorname{Zr}(1)$ & $77.0(2)$ \\
\hline $\mathrm{C}(10)-\operatorname{Zr}(1)-\mathrm{C}(14)$ & $32.79(11)$ & $C(6)-C(1)-\operatorname{Zr}(1)$ & $81.2(3)$ \\
\hline $\mathrm{C}(12)-\mathrm{Zr}(1)-\mathrm{C}(14)$ & $54.22(12)$ & $\mathrm{C}(3)-\mathrm{C}(2)-\mathrm{C}(1)$ & $121.3(4)$ \\
\hline$C(5)-\operatorname{Zr}(1)-C(14)$ & $148.15(13)$ & $C(3)-C(2)-\operatorname{Zr}(1)$ & $72.7(2)$ \\
\hline $\mathrm{C}(6)-\mathrm{Zr}(1)-\mathrm{C}(14)$ & 171.97(12) & $C(1)-C(2)-\operatorname{Zr}(1)$ & $69.1(2)$ \\
\hline $\mathrm{C}(10)-\mathrm{Si}(1)-\mathrm{C}(25)$ & $110.02(19)$ & $\mathrm{C}(2)-\mathrm{C}(3)-\mathrm{C}(4)$ & $121.9(4)$ \\
\hline $\mathrm{C}(10)-\mathrm{Si}(1)-\mathrm{C}(24)$ & $116.04(18)$ & $C(2)-C(3)-\operatorname{Zr}(1)$ & $74.4(2)$ \\
\hline $\mathrm{C}(25)-\mathrm{Si}(1)-\mathrm{C}(24)$ & $107.1(2)$ & $C(4)-C(3)-\operatorname{Zr}(1)$ & $68.7(2)$ \\
\hline $\mathrm{C}(10)-\mathrm{Si}(1)-\mathrm{C}(23)$ & $105.87(17)$ & $C(3)-C(4)-C(5)$ & $115.4(4)$ \\
\hline C(25)-Si(1)-C(23) & $107.84(19)$ & $C(3)-C(4)-\operatorname{Zr}(1)$ & $77.1(2)$ \\
\hline C(24)-Si(1)-C(23) & $109.74(18)$ & $C(5)-C(4)-\operatorname{Zr}(1)$ & $81.1(2)$ \\
\hline $\mathrm{C}(12)-\mathrm{Si}(2)-\mathrm{C}(27)$ & $116.79(17)$ & $\mathrm{C}(9)-\mathrm{C}(5)-\mathrm{C}(6)$ & $108.5(3)$ \\
\hline $\mathrm{C}(12)-\mathrm{Si}(2)-\mathrm{C}(28)$ & 109.96(17) & $\mathrm{C}(9)-\mathrm{C}(5)-\mathrm{C}(4)$ & $131.2(4)$ \\
\hline $\mathrm{C}(27)-\mathrm{Si}(2)-\mathrm{C}(28)$ & $106.64(18)$ & $\mathrm{C}(6)-\mathrm{C}(5)-\mathrm{C}(4)$ & $120.3(3)$ \\
\hline $\mathrm{C}(12)-\mathrm{Si}(2)-\mathrm{C}(26)$ & 107.13(19) & $C(9)-C(5)-\operatorname{Zr}(1)$ & $135.4(3)$ \\
\hline $\mathrm{C}(27)-\mathrm{Si}(2)-\mathrm{C}(26)$ & $108.0(2)$ & $C(6)-C(5)-\operatorname{Zr}(1)$ & $74.90(19)$ \\
\hline $\mathrm{C}(28)-\mathrm{Si}(2)-\mathrm{C}(26)$ & $108.00(17)$ & $C(4)-C(5)-\operatorname{Zr}(1)$ & $64.72(19)$ \\
\hline C(9)-Si(3)-C(29) & $110.41(18)$ & $C(5)-C(6)-C(7)$ & 109.7(3) \\
\hline $\mathrm{C}(9)-\mathrm{Si}(3)-\mathrm{C}(30)$ & $113.19(17)$ & $C(5)-C(6)-C(1)$ & $119.0(3)$ \\
\hline $\mathrm{C}(29)-\mathrm{Si}(3)-\mathrm{C}(30)$ & 109.77(18) & $C(7)-C(6)-C(1)$ & 131.3(4) \\
\hline $\mathrm{C}(9)-\mathrm{Si}(3)-\mathrm{C}(31)$ & $109.76(18)$ & $C(5)-C(6)-\operatorname{Zr}(1)$ & $72.8(2)$ \\
\hline $\mathrm{C}(29)-\mathrm{Si}(3)-\mathrm{C}(31)$ & 106.61(18) & $C(7)-C(6)-\operatorname{Zr}(1)$ & $136.8(3)$ \\
\hline $\mathrm{C}(30)-\mathrm{Si}(3)-\mathrm{C}(31)$ & $106.83(19)$ & $C(1)-C(6)-\operatorname{Zr}(1)$ & $64.9(2)$ \\
\hline$C(7)-S i(4)-C(32)$ & $111.62(17)$ & $\mathrm{C}(8)-\mathrm{C}(7)-\mathrm{C}(6)$ & 103.7(3) \\
\hline
\end{tabular}




\begin{tabular}{|c|c|c|c|}
\hline$C(8)-C(7)-S i(4)$ & $121.7(3)$ & $\mathrm{C}(17)-\mathrm{C}(18)-\mathrm{C}(13)$ & $119.3(4)$ \\
\hline$C(6)-C(7)-S i(4)$ & $134.3(3)$ & $\mathrm{O}(1)-\mathrm{C}(20)-\mathrm{C}(21)$ & $107.7(4)$ \\
\hline $\mathrm{C}(7)-\mathrm{C}(8)-\mathrm{C}(9)$ & $113.6(3)$ & $\mathrm{O}(2)-\mathrm{C}(21)-\mathrm{C}(20)$ & $107.7(4)$ \\
\hline$C(8)-C(9)-C(5)$ & $104.5(3)$ & $\mathrm{O}(1 \mathrm{~A})-\mathrm{Zr}(1 \mathrm{~A})-\mathrm{O}(2 \mathrm{~A})$ & $68.12(10)$ \\
\hline C(8)-C(9)-Si(3) & $122.8(3)$ & $\mathrm{O}(1 \mathrm{~A})-\mathrm{Zr}(1 \mathrm{~A})-\mathrm{C}(1 \mathrm{~A})$ & $89.38(13)$ \\
\hline$C(5)-C(9)-S i(3)$ & $132.6(3)$ & $\mathrm{O}(2 \mathrm{~A})-\mathrm{Zr}(1 \mathrm{~A})-\mathrm{C}(1 \mathrm{~A})$ & $130.36(11)$ \\
\hline$C(11)-C(10)-C(14)$ & $103.5(4)$ & $\mathrm{O}(1 \mathrm{~A})-\mathrm{Zr}(1 \mathrm{~A})-\mathrm{C}(4 \mathrm{~A})$ & $130.05(11)$ \\
\hline$C(11)-C(10)-\operatorname{Si}(1)$ & $122.7(3)$ & $\mathrm{O}(2 \mathrm{~A})-\mathrm{Zr}(1 \mathrm{~A})-\mathrm{C}(4 \mathrm{~A})$ & $86.35(13)$ \\
\hline$C(14)-C(10)-S i(1)$ & $128.3(3)$ & $C(1 \mathrm{~A})-\mathrm{Zr}(1 \mathrm{~A})-\mathrm{C}(4 \mathrm{~A})$ & $75.16(15)$ \\
\hline$C(11)-C(10)-\operatorname{Zr}(1)$ & $70.4(2)$ & $\mathrm{O}(1 \mathrm{~A})-\operatorname{Zr}(1 \mathrm{~A})-\mathrm{C}(11 \mathrm{~A})$ & $126.50(13)$ \\
\hline$C(14)-C(10)-Z r(1)$ & $78.7(2)$ & $\mathrm{O}(2 \mathrm{~A})-\mathrm{Zr}(1 \mathrm{~A})-\mathrm{C}(11 \mathrm{~A})$ & $125.68(12)$ \\
\hline $\operatorname{Si}(1)-C(10)-\operatorname{Zr}(1)$ & $135.16(17)$ & $C(1 \mathrm{~A})-\operatorname{Zr}(1 \mathrm{~A})-\mathrm{C}(11 \mathrm{~A})$ & $103.50(14)$ \\
\hline$C(10)-C(11)-C(12)$ & $114.4(4)$ & $C(4 \mathrm{~A})-\operatorname{Zr}(1 \mathrm{~A})-\mathrm{C}(11 \mathrm{~A})$ & $103.37(15)$ \\
\hline$C(10)-C(11)-\operatorname{Zr}(1)$ & $76.3(2)$ & $\mathrm{O}(1 \mathrm{~A})-\mathrm{Zr}(1 \mathrm{~A})-\mathrm{C}(2 \mathrm{~A})$ & $122.70(13)$ \\
\hline$C(12)-C(11)-\operatorname{Zr}(1)$ & $76.4(2)$ & $\mathrm{O}(2 \mathrm{~A})-\mathrm{Zr}(1 \mathrm{~A})-\mathrm{C}(2 \mathrm{~A})$ & $144.76(12)$ \\
\hline$C(11)-C(12)-C(13)$ & $103.5(3)$ & $C(1 \mathrm{~A})-\mathrm{Zr}(1 \mathrm{~A})-\mathrm{C}(2 \mathrm{~A})$ & $34.05(13)$ \\
\hline $\mathrm{C}(11)-\mathrm{C}(12)-\mathrm{Si}(2)$ & $122.6(3)$ & $\mathrm{C}(4 \mathrm{~A})-\mathrm{Zr}(1 \mathrm{~A})-\mathrm{C}(2 \mathrm{~A})$ & $60.71(15)$ \\
\hline$C(13)-C(12)-S i(2)$ & $129.5(3)$ & $C(11 \mathrm{~A})-\operatorname{Zr}(1 \mathrm{~A})-\mathrm{C}(2 \mathrm{~A})$ & $77.55(14)$ \\
\hline$C(11)-C(12)-\operatorname{Zr}(1)$ & $70.1(2)$ & $\mathrm{O}(1 \mathrm{~A})-\mathrm{Zr}(1 \mathrm{~A})-\mathrm{C}(3 \mathrm{~A})$ & $147.52(12)$ \\
\hline$C(13)-C(12)-\operatorname{Zr}(1)$ & $78.8(2)$ & $\mathrm{O}(2 \mathrm{~A})-\mathrm{Zr}(1 \mathrm{~A})-\mathrm{C}(3 \mathrm{~A})$ & $119.13(13)$ \\
\hline $\mathrm{Si}(2)-\mathrm{C}(12)-\mathrm{Zr}(1)$ & $132.98(17)$ & $C(1 \mathrm{~A})-\mathrm{Zr}(1 \mathrm{~A})-\mathrm{C}(3 \mathrm{~A})$ & $61.32(15)$ \\
\hline$C(18)-C(13)-C(14)$ & $119.0(4)$ & $C(4 \mathrm{~A})-\mathrm{Zr}(1 \mathrm{~A})-\mathrm{C}(3 \mathrm{~A})$ & $33.81(13)$ \\
\hline$C(18)-C(13)-C(12)$ & $131.8(4)$ & $C(11 \mathrm{~A})-\operatorname{Zr}(1 \mathrm{~A})-\mathrm{C}(3 \mathrm{~A})$ & $77.30(14)$ \\
\hline$C(14)-C(13)-C(12)$ & $109.2(3)$ & $C(2 \mathrm{~A})-\mathrm{Zr}(1 \mathrm{~A})-\mathrm{C}(3 \mathrm{~A})$ & $32.87(13)$ \\
\hline$C(18)-C(13)-\operatorname{Zr}(1)$ & $124.6(2)$ & $\mathrm{O}(1 \mathrm{~A})-\mathrm{Zr}(1 \mathrm{~A})-\mathrm{C}(10 \mathrm{~A})$ & $122.64(11)$ \\
\hline$C(14)-C(13)-\operatorname{Zr}(1)$ & $74.0(2)$ & $\mathrm{O}(2 \mathrm{~A})-\mathrm{Zr}(1 \mathrm{~A})-\mathrm{C}(10 \mathrm{~A})$ & $91.99(11)$ \\
\hline$C(12)-C(13)-\operatorname{Zr}(1)$ & $68.70(19)$ & $C(1 \mathrm{~A})-\operatorname{Zr}(1 \mathrm{~A})-\mathrm{C}(10 \mathrm{~A})$ & $135.84(13)$ \\
\hline$C(15)-C(14)-C(13)$ & $118.9(4)$ & $C(4 A)-Z r(1 A)-C(10 A)$ & $99.43(13)$ \\
\hline$C(15)-C(14)-C(10)$ & $131.9(4)$ & $C(11 \mathrm{~A})-\operatorname{Zr}(1 \mathrm{~A})-\mathrm{C}(10 \mathrm{~A})$ & $33.95(12)$ \\
\hline$C(13)-C(14)-C(10)$ & $109.2(4)$ & $C(2 A)-Z r(1 A)-C(10 A)$ & $104.69(13)$ \\
\hline$C(15)-C(14)-\operatorname{Zr}(1)$ & $125.9(2)$ & $C(3 \mathrm{~A})-\operatorname{Zr}(1 \mathrm{~A})-\mathrm{C}(10 \mathrm{~A})$ & $89.60(13)$ \\
\hline$C(13)-C(14)-\operatorname{Zr}(1)$ & $74.5(2)$ & $\mathrm{O}(1 \mathrm{~A})-\operatorname{Zr}(1 \mathrm{~A})-\mathrm{C}(12 \mathrm{~A})$ & $95.50(11)$ \\
\hline$C(10)-C(14)-\operatorname{Zr}(1)$ & $68.54(19)$ & $\mathrm{O}(2 \mathrm{~A})-\mathrm{Zr}(1 \mathrm{~A})-\mathrm{C}(12 \mathrm{~A})$ & $130.58(11)$ \\
\hline$C(16)-C(15)-C(14)$ & $121.6(5)$ & $C(1 \mathrm{~A})-\operatorname{Zr}(1 \mathrm{~A})-\mathrm{C}(12 \mathrm{~A})$ & $93.71(13)$ \\
\hline$C(15)-C(16)-C(17)$ & $119.2(5)$ & $C(4 A)-\operatorname{Zr}(1 \mathrm{~A})-\mathrm{C}(12 \mathrm{~A})$ & $132.00(13)$ \\
\hline$C(18)-C(17)-C(16)$ & $122.1(5)$ & $C(11 A)-Z r(1 A)-C(12 A)$ & $33.13(12)$ \\
\hline
\end{tabular}




\begin{tabular}{|c|c|c|c|}
\hline $\mathrm{C}(2 \mathrm{~A})-\mathrm{Zr}(1 \mathrm{~A})-\mathrm{C}(12 \mathrm{~A})$ & $83.90(13)$ & $\mathrm{C}(12 \mathrm{~A})-\mathrm{Si}(1 \mathrm{~A})-\mathrm{C}(24 \mathrm{~A})$ & $114.45(18)$ \\
\hline$C(3 \mathrm{~A})-\mathrm{Zr}(1 \mathrm{~A})-\mathrm{C}(12 \mathrm{~A})$ & $99.82(13)$ & $C(23 A)-S i(1 A)-C(24 A)$ & $107.4(2)$ \\
\hline$C(10 A)-\operatorname{Zr}(1 A)-C(12 A)$ & $57.02(12)$ & $C(25 A)-S i(1 A)-C(24 A)$ & $107.4(2)$ \\
\hline $\mathrm{O}(1 \mathrm{~A})-\mathrm{Zr}(1 \mathrm{~A})-\mathrm{C}(6 \mathrm{~A})$ & $79.76(11)$ & $\mathrm{C}(10 \mathrm{~A})-\mathrm{Si}(2 \mathrm{~A})-\mathrm{C}(28 \mathrm{~A})$ & $106.70(17)$ \\
\hline $\mathrm{O}(2 \mathrm{~A})-\mathrm{Zr}(1 \mathrm{~A})-\mathrm{C}(6 \mathrm{~A})$ & $96.74(10)$ & $C(10 A)-S i(2 A)-C(26 A)$ & $110.65(18)$ \\
\hline$C(1 \mathrm{~A})-\operatorname{Zr}(1 \mathrm{~A})-\mathrm{C}(6 \mathrm{~A})$ & $34.20(12)$ & $\mathrm{C}(28 \mathrm{~A})-\mathrm{Si}(2 \mathrm{~A})-\mathrm{C}(26 \mathrm{~A})$ & $109.2(2)$ \\
\hline$C(4 A)-Z r(1 A)-C(6 A)$ & $60.78(13)$ & $C(10 A)-S i(2 A)-C(27 A)$ & $115.85(18)$ \\
\hline$C(11 \mathrm{~A})-\operatorname{Zr}(1 \mathrm{~A})-\mathrm{C}(6 \mathrm{~A})$ & $134.95(13)$ & $\mathrm{C}(28 \mathrm{~A})-\mathrm{Si}(2 \mathrm{~A})-\mathrm{C}(27 \mathrm{~A})$ & $105.7(2)$ \\
\hline$C(2 A)-\operatorname{Zr}(1 \mathrm{~A})-\mathrm{C}(6 \mathrm{~A})$ & $57.69(12)$ & $C(26 A)-S i(2 A)-C(27 A)$ & $108.5(2)$ \\
\hline$C(3 \mathrm{~A})-\operatorname{Zr}(1 \mathrm{~A})-\mathrm{C}(6 \mathrm{~A})$ & $68.13(13)$ & $C(7 A)-\operatorname{Si}(3 A)-C(30 A)$ & $113.67(17)$ \\
\hline$C(10 A)-Z r(1 A)-C(6 A)$ & $157.59(13)$ & $C(7 A)-\operatorname{Si}(3 A)-C(29 A)$ & $111.76(18)$ \\
\hline$C(12 \mathrm{~A})-\mathrm{Zr}(1 \mathrm{~A})-\mathrm{C}(6 \mathrm{~A})$ & $127.03(12)$ & $C(30 A)-S i(3 A)-C(29 A)$ & $108.55(19)$ \\
\hline $\mathrm{O}(1 \mathrm{~A})-\mathrm{Zr}(1 \mathrm{~A})-\mathrm{C}(5 \mathrm{~A})$ & $97.17(10)$ & $C(7 A)-\operatorname{Si}(3 A)-C(31 A)$ & $109.73(18)$ \\
\hline $\mathrm{O}(2 \mathrm{~A})-\mathrm{Zr}(1 \mathrm{~A})-\mathrm{C}(5 \mathrm{~A})$ & $78.08(10)$ & $C(30 A)-S i(3 A)-C(31 A)$ & $106.78(19)$ \\
\hline$C(1 A)-\operatorname{Zr}(1 A)-C(5 A)$ & $60.90(13)$ & $C(29 A)-S i(3 A)-C(31 A)$ & $106.0(2)$ \\
\hline$C(4 A)-Z r(1 A)-C(5 A)$ & $33.93(12)$ & $C(9 A)-S i(4 A)-C(33 A)$ & $112.0(2)$ \\
\hline$C(11 \mathrm{~A})-\mathrm{Zr}(1 \mathrm{~A})-\mathrm{C}(5 \mathrm{~A})$ & $134.65(14)$ & $\mathrm{C}(9 \mathrm{~A})-\mathrm{Si}(4 \mathrm{~A})-\mathrm{C}(32 \mathrm{~A})$ & $110.02(17)$ \\
\hline$C(2 A)-\operatorname{Zr}(1 A)-C(5 A)$ & $67.62(12)$ & $C(33 A)-S i(4 A)-C(32 A)$ & $105.55(18)$ \\
\hline$C(3 A)-\operatorname{Zr}(1 \mathrm{~A})-\mathrm{C}(5 \mathrm{~A})$ & $57.63(12)$ & $\mathrm{C}(9 \mathrm{~A})-\mathrm{Si}(4 \mathrm{~A})-\mathrm{C}(34 \mathrm{~A})$ & $113.60(18)$ \\
\hline$C(10 \mathrm{~A})-\mathrm{Zr}(1 \mathrm{~A})-\mathrm{C}(5 \mathrm{~A})$ & $131.97(12)$ & $C(33 \mathrm{~A})-\mathrm{Si}(4 \mathrm{~A})-\mathrm{C}(34 \mathrm{~A})$ & $108.1(2)$ \\
\hline$C(12 A)-Z r(1 A)-C(5 A)$ & $151.34(12)$ & $\mathrm{C}(32 \mathrm{~A})-\mathrm{Si}(4 \mathrm{~A})-\mathrm{C}(34 \mathrm{~A})$ & $107.14(19)$ \\
\hline$C(6 A)-\operatorname{Zr}(1 \mathrm{~A})-\mathrm{C}(5 \mathrm{~A})$ & $32.49(11)$ & $\mathrm{C}\left(20^{\prime}\right)-\mathrm{O}(1 \mathrm{~A})-\mathrm{C}(19 \mathrm{~A})$ & $108.6(8)$ \\
\hline $\mathrm{O}(1 \mathrm{~A})-\mathrm{Zr}(1 \mathrm{~A})-\mathrm{C}(14 \mathrm{~A})$ & $89.59(11)$ & $\mathrm{C}\left(20^{\prime}\right)-\mathrm{O}(1 \mathrm{~A})-\mathrm{C}(20 \mathrm{~A})$ & $26.5(7)$ \\
\hline $\mathrm{O}(2 \mathrm{~A})-\mathrm{Zr}(1 \mathrm{~A})-\mathrm{C}(14 \mathrm{~A})$ & $78.74(10)$ & $\mathrm{C}(19 \mathrm{~A})-\mathrm{O}(1 \mathrm{~A})-\mathrm{C}(20 \mathrm{~A})$ & $116.8(8)$ \\
\hline $\mathrm{C}(1 \mathrm{~A})-\mathrm{Zr}(1 \mathrm{~A})-\mathrm{C}(14 \mathrm{~A})$ & $147.36(13)$ & $\mathrm{C}\left(20^{\prime}\right)-\mathrm{O}(1 \mathrm{~A})-\mathrm{Zr}(1 \mathrm{~A})$ & $125.5(8)$ \\
\hline $\mathrm{C}(4 \mathrm{~A})-\mathrm{Zr}(1 \mathrm{~A})-\mathrm{C}(14 \mathrm{~A})$ & $127.86(13)$ & $\mathrm{C}(19 \mathrm{~A})-\mathrm{O}(1 \mathrm{~A})-\mathrm{Zr}(1 \mathrm{~A})$ & $125.8(2)$ \\
\hline$C(11 \mathrm{~A})-\operatorname{Zr}(1 \mathrm{~A})-\mathrm{C}(14 \mathrm{~A})$ & $52.94(13)$ & $\mathrm{C}(20 \mathrm{~A})-\mathrm{O}(1 \mathrm{~A})-\mathrm{Zr}(1 \mathrm{~A})$ & $113.6(8)$ \\
\hline $\mathrm{C}(2 \mathrm{~A})-\mathrm{Zr}(1 \mathrm{~A})-\mathrm{C}(14 \mathrm{~A})$ & $130.40(12)$ & $\mathrm{C}(21 \mathrm{~A})-\mathrm{O}(2 \mathrm{~A})-\mathrm{C}(22 \mathrm{~A})$ & $111.4(10)$ \\
\hline$C(3 A)-\operatorname{Zr}(1 \mathrm{~A})-C(14 \mathrm{~A})$ & $122.51(13)$ & $\mathrm{C}(21 \mathrm{~A})-\mathrm{O}(2 \mathrm{~A})-\mathrm{C}\left(21^{\prime}\right)$ & $23.2(8)$ \\
\hline$C(10 A)-\operatorname{Zr}(1 \mathrm{~A})-\mathrm{C}(14 \mathrm{~A})$ & $33.05(11)$ & $\mathrm{C}(22 \mathrm{~A})-\mathrm{O}(2 \mathrm{~A})-\mathrm{C}\left(21^{\prime}\right)$ & $115.8(10)$ \\
\hline $\mathrm{C}(12 \mathrm{~A})-\mathrm{Zr}(1 \mathrm{~A})-\mathrm{C}(14 \mathrm{~A})$ & $53.95(12)$ & $\mathrm{C}(21 \mathrm{~A})-\mathrm{O}(2 \mathrm{~A})-\mathrm{Zr}(1 \mathrm{~A})$ & $122.9(10)$ \\
\hline $\mathrm{C}(6 \mathrm{~A})-\mathrm{Zr}(1 \mathrm{~A})-\mathrm{C}(14 \mathrm{~A})$ & $169.35(12)$ & $\mathrm{C}(22 \mathrm{~A})-\mathrm{O}(2 \mathrm{~A})-\mathrm{Zr}(1 \mathrm{~A})$ & $125.7(3)$ \\
\hline$C(5 \mathrm{~A})-\mathrm{Zr}(1 \mathrm{~A})-\mathrm{C}(14 \mathrm{~A})$ & $151.33(12)$ & $\mathrm{C}\left(21^{\prime}\right)-\mathrm{O}(2 \mathrm{~A})-\mathrm{Zr}(1 \mathrm{~A})$ & $113.9(10)$ \\
\hline$C(12 A)-\operatorname{Si}(1 \mathrm{~A})-\mathrm{C}(23 \mathrm{~A})$ & $106.64(18)$ & $C(2 A)-C(1 A)-C(6 A)$ & $115.1(4)$ \\
\hline$C(12 A)-\operatorname{Si}(1 A)-C(25 A)$ & $110.9(2)$ & $C(2 A)-C(1 A)-Z r(1 A)$ & $77.1(2)$ \\
\hline$C(23 A)-S i(1 A)-C(25 A)$ & 110.01(19) & $C(6 \mathrm{~A})-\mathrm{C}(1 \mathrm{~A})-\mathrm{Zr}(1 \mathrm{~A})$ & $80.4(2)$ \\
\hline
\end{tabular}




\begin{tabular}{|c|c|c|c|}
\hline $\mathrm{C}(3 \mathrm{~A})-\mathrm{C}(2 \mathrm{~A})-\mathrm{C}(1 \mathrm{~A})$ & $122.4(4)$ & $\mathrm{C}(10 \mathrm{~A})-\mathrm{C}(11 \mathrm{~A})-\operatorname{Zr}(1 \mathrm{~A})$ & $75.1(2)$ \\
\hline$C(3 A)-C(2 A)-Z r(1 A)$ & $73.8(2)$ & $\mathrm{C}(11 \mathrm{~A})-\mathrm{C}(12 \mathrm{~A})-\mathrm{C}(13 \mathrm{~A})$ & $104.0(4)$ \\
\hline$C(1 \mathrm{~A})-\mathrm{C}(2 \mathrm{~A})-\mathrm{Zr}(1 \mathrm{~A})$ & $68.9(2)$ & $\mathrm{C}(11 \mathrm{~A})-\mathrm{C}(12 \mathrm{~A})-\mathrm{Si}(1 \mathrm{~A})$ & $126.1(3)$ \\
\hline $\mathrm{C}(2 \mathrm{~A})-\mathrm{C}(3 \mathrm{~A})-\mathrm{C}(4 \mathrm{~A})$ & $121.1(4)$ & $\mathrm{C}(13 \mathrm{~A})-\mathrm{C}(12 \mathrm{~A})-\mathrm{Si}(1 \mathrm{~A})$ & $125.1(3)$ \\
\hline$C(2 A)-C(3 A)-Z r(1 A)$ & 73.3(3) & $\mathrm{C}(11 \mathrm{~A})-\mathrm{C}(12 \mathrm{~A})-\operatorname{Zr}(1 \mathrm{~A})$ & $69.3(2)$ \\
\hline $\mathrm{C}(4 \mathrm{~A})-\mathrm{C}(3 \mathrm{~A})-\mathrm{Zr}(1 \mathrm{~A})$ & $69.2(2)$ & $C(13 \mathrm{~A})-\mathrm{C}(12 \mathrm{~A})-\mathrm{Zr}(1 \mathrm{~A})$ & $79.8(2)$ \\
\hline$C(3 A)-C(4 A)-C(5 A)$ & $116.3(4)$ & $\mathrm{Si}(1 \mathrm{~A})-\mathrm{C}(12 \mathrm{~A})-\operatorname{Zr}(1 \mathrm{~A})$ & $134.43(17)$ \\
\hline$C(3 A)-C(4 A)-Z r(1 A)$ & $77.0(3)$ & $C(18 \mathrm{~A})-\mathrm{C}(13 \mathrm{~A})-\mathrm{C}(14 \mathrm{~A})$ & $119.4(4)$ \\
\hline$C(5 A)-C(4 A)-Z r(1 A)$ & $81.2(3)$ & $\mathrm{C}(18 \mathrm{~A})-\mathrm{C}(13 \mathrm{~A})-\mathrm{C}(12 \mathrm{~A})$ & $131.4(4)$ \\
\hline$C(9 A)-C(5 A)-C(6 A)$ & $109.0(3)$ & $C(14 A)-C(13 A)-C(12 A)$ & $109.1(4)$ \\
\hline $\mathrm{C}(9 \mathrm{~A})-\mathrm{C}(5 \mathrm{~A})-\mathrm{C}(4 \mathrm{~A})$ & $132.0(4)$ & $\mathrm{C}(18 \mathrm{~A})-\mathrm{C}(13 \mathrm{~A})-\mathrm{Zr}(1 \mathrm{~A})$ & $128.6(3)$ \\
\hline$C(6 A)-C(5 A)-C(4 A)$ & $119.0(3)$ & $\mathrm{C}(14 \mathrm{~A})-\mathrm{C}(13 \mathrm{~A})-\operatorname{Zr}(1 \mathrm{~A})$ & $72.3(2)$ \\
\hline$C(9 A)-C(5 A)-Z r(1 A)$ & $136.4(3)$ & $\mathrm{C}(12 \mathrm{~A})-\mathrm{C}(13 \mathrm{~A})-\mathrm{Zr}(1 \mathrm{~A})$ & $68.2(2)$ \\
\hline$C(6 A)-C(5 A)-Z r(1 A)$ & 72.71(19) & $C(15 A)-C(14 A)-C(13 A)$ & $117.8(4)$ \\
\hline$C(4 A)-C(5 A)-Z r(1 A)$ & $64.9(2)$ & $C(15 A)-C(14 A)-C(10 A)$ & $132.5(4)$ \\
\hline$C(5 A)-C(6 A)-C(7 A)$ & $108.6(3)$ & $C(13 A)-C(14 A)-C(10 A)$ & $109.6(4)$ \\
\hline$C(5 A)-C(6 A)-C(1 A)$ & $120.3(3)$ & $C(15 A)-C(14 A)-Z r(1 A)$ & $123.8(2)$ \\
\hline$C(7 A)-C(6 A)-C(1 A)$ & 131.1(4) & $\mathrm{C}(13 \mathrm{~A})-\mathrm{C}(14 \mathrm{~A})-\mathrm{Zr}(1 \mathrm{~A})$ & $76.5(2)$ \\
\hline$C(5 A)-C(6 A)-Z r(1 A)$ & $74.80(19)$ & $\mathrm{C}(10 \mathrm{~A})-\mathrm{C}(14 \mathrm{~A})-\mathrm{Zr}(1 \mathrm{~A})$ & $67.86(19)$ \\
\hline$C(7 A)-C(6 A)-Z r(1 A)$ & $135.4(3)$ & $C(16 A)-C(15 A)-C(14 A)$ & $120.4(5)$ \\
\hline$C(1 \mathrm{~A})-\mathrm{C}(6 \mathrm{~A})-\mathrm{Zr}(1 \mathrm{~A})$ & $65.4(2)$ & $C(15 A)-C(16 A)-C(17 A)$ & $121.2(5)$ \\
\hline $\mathrm{C}(8 \mathrm{~A})-\mathrm{C}(7 \mathrm{~A})-\mathrm{C}(6 \mathrm{~A})$ & 104.3(3) & $C(18 A)-C(17 A)-C(16 A)$ & $119.8(5)$ \\
\hline C(8A)-C(7A)-Si(3A) & $123.1(3)$ & $\mathrm{C}(17 \mathrm{~A})-\mathrm{C}(18 \mathrm{~A})-\mathrm{C}(13 \mathrm{~A})$ & $121.1(5)$ \\
\hline $\mathrm{C}(6 \mathrm{~A})-\mathrm{C}(7 \mathrm{~A})-\mathrm{Si}(3 \mathrm{~A})$ & $132.0(3)$ & $\mathrm{C}(21 \mathrm{~A})-\mathrm{C}(20 \mathrm{~A})-\mathrm{O}(1 \mathrm{~A})$ & $109.6(14)$ \\
\hline $\mathrm{C}(7 \mathrm{~A})-\mathrm{C}(8 \mathrm{~A})-\mathrm{C}(9 \mathrm{~A})$ & $113.7(3)$ & $\mathrm{O}(1 \mathrm{~A})-\mathrm{C}\left(20^{\prime}\right)-\mathrm{C}\left(21^{\prime}\right)$ & $105.4(15)$ \\
\hline $\mathrm{C}(8 \mathrm{~A})-\mathrm{C}(9 \mathrm{~A})-\mathrm{C}(5 \mathrm{~A})$ & 104.3(3) & $\mathrm{O}(2 \mathrm{~A})-\mathrm{C}(21 \mathrm{~A})-\mathrm{C}(20 \mathrm{~A})$ & $108.9(16)$ \\
\hline $\mathrm{C}(8 \mathrm{~A})-\mathrm{C}(9 \mathrm{~A})-\mathrm{Si}(4 \mathrm{~A})$ & $122.1(3)$ & $\mathrm{C}\left(20^{\prime}\right)-\mathrm{C}\left(21^{\prime}\right)-\mathrm{O}(2 \mathrm{~A})$ & $106.7(15)$ \\
\hline$C(5 A)-C(9 A)-S i(4 A)$ & $132.3(3)$ & $\mathrm{O}(1 \mathrm{~B})-\mathrm{Zr}(1 \mathrm{~B})-\mathrm{O}(2 \mathrm{~B})$ & $68.52(10)$ \\
\hline$C(11 A)-C(10 A)-C(14 A)$ & $102.8(3)$ & $\mathrm{O}(1 \mathrm{~B})-\mathrm{Zr}(1 \mathrm{~B})-\mathrm{C}(4 \mathrm{~B})$ & $91.06(14)$ \\
\hline $\mathrm{C}(11 \mathrm{~A})-\mathrm{C}(10 \mathrm{~A})-\mathrm{Si}(2 \mathrm{~A})$ & $123.1(3)$ & $\mathrm{O}(2 \mathrm{~B})-\mathrm{Zr}(1 \mathrm{~B})-\mathrm{C}(4 \mathrm{~B})$ & $131.77(12)$ \\
\hline $\mathrm{C}(14 \mathrm{~A})-\mathrm{C}(10 \mathrm{~A})-\mathrm{Si}(2 \mathrm{~A})$ & $127.3(3)$ & $\mathrm{O}(1 \mathrm{~B})-\mathrm{Zr}(1 \mathrm{~B})-\mathrm{C}(1 \mathrm{~B})$ & $128.36(11)$ \\
\hline$C(11 \mathrm{~A})-\mathrm{C}(10 \mathrm{~A})-\mathrm{Zr}(1 \mathrm{~A})$ & $70.9(2)$ & $\mathrm{O}(2 \mathrm{~B})-\mathrm{Zr}(1 \mathrm{~B})-\mathrm{C}(1 \mathrm{~B})$ & $84.63(12)$ \\
\hline $\mathrm{C}(14 \mathrm{~A})-\mathrm{C}(10 \mathrm{~A})-\mathrm{Zr}(1 \mathrm{~A})$ & $79.1(2)$ & $\mathrm{C}(4 \mathrm{~B})-\mathrm{Zr}(1 \mathrm{~B})-\mathrm{C}(1 \mathrm{~B})$ & $74.70(15)$ \\
\hline $\operatorname{Si}(2 \mathrm{~A})-\mathrm{C}(10 \mathrm{~A})-\operatorname{Zr}(1 \mathrm{~A})$ & $136.48(18)$ & $\mathrm{O}(1 \mathrm{~B})-\mathrm{Zr}(1 \mathrm{~B})-\mathrm{C}(11 \mathrm{~B})$ & $125.27(13)$ \\
\hline$C(12 A)-C(11 A)-C(10 A)$ & $114.2(4)$ & $\mathrm{O}(2 \mathrm{~B})-\mathrm{Zr}(1 \mathrm{~B})-\mathrm{C}(11 \mathrm{~B})$ & $126.59(12)$ \\
\hline$C(12 A)-C(11 A)-Z r(1 A)$ & $77.5(2)$ & C(4B)-Zr(1B)-C(11B) & $101.14(14)$ \\
\hline
\end{tabular}




\begin{tabular}{|c|c|c|c|}
\hline $\mathrm{C}(1 \mathrm{~B})-\mathrm{Zr}(1 \mathrm{~B})-\mathrm{C}(11 \mathrm{~B})$ & $106.25(15)$ & $\mathrm{O}(1 \mathrm{~B})-\mathrm{Zr}(1 \mathrm{~B})-\mathrm{C}(6 \mathrm{~B})$ & $95.82(11)$ \\
\hline $\mathrm{O}(1 \mathrm{~B})-\mathrm{Zr}(1 \mathrm{~B})-\mathrm{C}(3 \mathrm{~B})$ & $124.84(13)$ & $\mathrm{O}(2 \mathrm{~B})-\mathrm{Zr}(1 \mathrm{~B})-\mathrm{C}(6 \mathrm{~B})$ & $78.13(10)$ \\
\hline $\mathrm{O}(2 \mathrm{~B})-\mathrm{Zr}(1 \mathrm{~B})-\mathrm{C}(3 \mathrm{~B})$ & $143.79(12)$ & $\mathrm{C}(4 \mathrm{~B})-\mathrm{Zr}(1 \mathrm{~B})-\mathrm{C}(6 \mathrm{~B})$ & $60.38(13)$ \\
\hline $\mathrm{C}(4 \mathrm{~B})-\mathrm{Zr}(1 \mathrm{~B})-\mathrm{C}(3 \mathrm{~B})$ & $34.30(14)$ & $\mathrm{C}(1 \mathrm{~B})-\mathrm{Zr}(1 \mathrm{~B})-\mathrm{C}(6 \mathrm{~B})$ & $34.02(13)$ \\
\hline$C(1 B)-Z r(1 B)-C(3 B)$ & $60.47(14)$ & $\mathrm{C}(11 \mathrm{~B})-\mathrm{Zr}(1 \mathrm{~B})-\mathrm{C}(6 \mathrm{~B})$ & $136.49(14)$ \\
\hline $\mathrm{C}(11 \mathrm{~B})-\mathrm{Zr}(1 \mathrm{~B})-\mathrm{C}(3 \mathrm{~B})$ & $76.37(13)$ & $C(3 B)-Z r(1 B)-C(6 B)$ & $67.61(12)$ \\
\hline $\mathrm{O}(1 \mathrm{~B})-\mathrm{Zr}(1 \mathrm{~B})-\mathrm{C}(2 \mathrm{~B})$ & $147.77(12)$ & $C(2 B)-Z r(1 B)-C(6 B)$ & $57.37(13)$ \\
\hline $\mathrm{O}(2 \mathrm{~B})-\mathrm{Zr}(1 \mathrm{~B})-\mathrm{C}(2 \mathrm{~B})$ & $116.56(13)$ & $\mathrm{C}(12 \mathrm{~B})-\mathrm{Zr}(1 \mathrm{~B})-\mathrm{C}(6 \mathrm{~B})$ & $134.99(13)$ \\
\hline $\mathrm{C}(4 \mathrm{~B})-\mathrm{Zr}(1 \mathrm{~B})-\mathrm{C}(2 \mathrm{~B})$ & $61.20(15)$ & $\mathrm{C}(10 \mathrm{~B})-\mathrm{Zr}(1 \mathrm{~B})-\mathrm{C}(6 \mathrm{~B})$ & $150.92(12)$ \\
\hline $\mathrm{C}(1 \mathrm{~B})-\mathrm{Zr}(1 \mathrm{~B})-\mathrm{C}(2 \mathrm{~B})$ & $33.39(13)$ & $C(5 B)-Z r(1 B)-C(6 B)$ & $32.27(11)$ \\
\hline $\mathrm{C}(11 \mathrm{~B})-\mathrm{Zr}(1 \mathrm{~B})-\mathrm{C}(2 \mathrm{~B})$ & $79.12(14)$ & $\mathrm{O}(1 \mathrm{~B})-\mathrm{Zr}(1 \mathrm{~B})-\mathrm{C}(13 \mathrm{~B})$ & $89.69(11)$ \\
\hline $\mathrm{C}(3 \mathrm{~B})-\mathrm{Zr}(1 \mathrm{~B})-\mathrm{C}(2 \mathrm{~B})$ & $32.87(13)$ & $\mathrm{O}(2 \mathrm{~B})-\mathrm{Zr}(1 \mathrm{~B})-\mathrm{C}(13 \mathrm{~B})$ & $79.49(10)$ \\
\hline $\mathrm{O}(1 \mathrm{~B})-\mathrm{Zr}(1 \mathrm{~B})-\mathrm{C}(12 \mathrm{~B})$ & $122.18(11)$ & $C(4 B)-Z r(1 B)-C(13 B)$ & $146.05(13)$ \\
\hline $\mathrm{O}(2 \mathrm{~B})-\mathrm{Zr}(1 \mathrm{~B})-\mathrm{C}(12 \mathrm{~B})$ & $93.23(11)$ & $\mathrm{C}(1 \mathrm{~B})-\mathrm{Zr}(1 \mathrm{~B})-\mathrm{C}(13 \mathrm{~B})$ & $128.85(13)$ \\
\hline $\mathrm{C}(4 \mathrm{~B})-\mathrm{Zr}(1 \mathrm{~B})-\mathrm{C}(12 \mathrm{~B})$ & $133.12(14)$ & $C(11 B)-Z r(1 B)-C(13 B)$ & $52.59(12)$ \\
\hline $\mathrm{C}(1 \mathrm{~B})-\mathrm{Zr}(1 \mathrm{~B})-\mathrm{C}(12 \mathrm{~B})$ & $101.81(13)$ & $C(3 B)-Z r(1 B)-C(13 B)$ & $128.96(13)$ \\
\hline $\mathrm{C}(11 \mathrm{~B})-\mathrm{Zr}(1 \mathrm{~B})-\mathrm{C}(12 \mathrm{~B})$ & $33.64(12)$ & $\mathrm{C}(2 \mathrm{~B})-\mathrm{Zr}(1 \mathrm{~B})-\mathrm{C}(13 \mathrm{~B})$ & $122.40(13)$ \\
\hline $\mathrm{C}(3 \mathrm{~B})-\mathrm{Zr}(1 \mathrm{~B})-\mathrm{C}(12 \mathrm{~B})$ & $102.54(14)$ & $C(12 B)-\operatorname{Zr}(1 B)-C(13 B)$ & $32.50(11)$ \\
\hline $\mathrm{C}(2 \mathrm{~B})-\mathrm{Zr}(1 \mathrm{~B})-\mathrm{C}(12 \mathrm{~B})$ & 89.91(13) & $C(10 B)-Z r(1 B)-C(13 B)$ & $53.96(11)$ \\
\hline $\mathrm{O}(1 \mathrm{~B})-\mathrm{Zr}(1 \mathrm{~B})-\mathrm{C}(10 \mathrm{~B})$ & $93.84(11)$ & $C(5 B)-Z r(1 B)-C(13 B)$ & $169.51(13)$ \\
\hline $\mathrm{O}(2 \mathrm{~B})-\mathrm{Zr}(1 \mathrm{~B})-\mathrm{C}(10 \mathrm{~B})$ & $130.83(10)$ & $\mathrm{C}(6 \mathrm{~B})-\mathrm{Zr}(1 \mathrm{~B})-\mathrm{C}(13 \mathrm{~B})$ & $153.10(12)$ \\
\hline $\mathrm{C}(4 \mathrm{~B})-\mathrm{Zr}(1 \mathrm{~B})-\mathrm{C}(10 \mathrm{~B})$ & $92.13(13)$ & $C(25 B)-S i(1 B)-C(10 B)$ & $110.27(19)$ \\
\hline $\mathrm{C}(1 \mathrm{~B})-\mathrm{Zr}(1 \mathrm{~B})-\mathrm{C}(10 \mathrm{~B})$ & $135.09(14)$ & $C(25 B)-S i(1 B)-C(23 B)$ & $108.68(18)$ \\
\hline $\mathrm{C}(11 \mathrm{~B})-\mathrm{Zr}(1 \mathrm{~B})-\mathrm{C}(10 \mathrm{~B})$ & $33.42(12)$ & $C(10 B)-S i(1 B)-C(23 B)$ & $106.19(17)$ \\
\hline $\mathrm{C}(3 \mathrm{~B})-\mathrm{Zr}(1 \mathrm{~B})-\mathrm{C}(10 \mathrm{~B})$ & $84.39(13)$ & $C(25 B)-S i(1 B)-C(24 B)$ & $106.92(19)$ \\
\hline $\mathrm{C}(2 \mathrm{~B})-\mathrm{Zr}(1 \mathrm{~B})-\mathrm{C}(10 \mathrm{~B})$ & $102.68(13)$ & $C(10 B)-S i(1 B)-C(24 B)$ & $116.70(18)$ \\
\hline $\mathrm{C}(12 \mathrm{~B})-\mathrm{Zr}(1 \mathrm{~B})-\mathrm{C}(10 \mathrm{~B})$ & $56.90(12)$ & $C(23 B)-S i(1 B)-C(24 B)$ & $107.86(19)$ \\
\hline $\mathrm{O}(1 \mathrm{~B})-\mathrm{Zr}(1 \mathrm{~B})-\mathrm{C}(5 \mathrm{~B})$ & $79.98(11)$ & $\mathrm{C}(26 \mathrm{~B})-\mathrm{Si}(2 \mathrm{~B})-\mathrm{C}(28 \mathrm{~B})$ & $110.2(2)$ \\
\hline $\mathrm{O}(2 \mathrm{~B})-\mathrm{Zr}(1 \mathrm{~B})-\mathrm{C}(5 \mathrm{~B})$ & $98.21(10)$ & $\mathrm{C}(26 \mathrm{~B})-\mathrm{Si}(2 \mathrm{~B})-\mathrm{C}(12 \mathrm{~B})$ & $111.23(19)$ \\
\hline $\mathrm{C}(4 \mathrm{~B})-\mathrm{Zr}(1 \mathrm{~B})-\mathrm{C}(5 \mathrm{~B})$ & $33.82(12)$ & $\mathrm{C}(28 \mathrm{~B})-\mathrm{Si}(2 \mathrm{~B})-\mathrm{C}(12 \mathrm{~B})$ & $106.10(18)$ \\
\hline $\mathrm{C}(1 \mathrm{~B})-\mathrm{Zr}(1 \mathrm{~B})-\mathrm{C}(5 \mathrm{~B})$ & $60.59(13)$ & $\mathrm{C}(26 \mathrm{~B})-\mathrm{Si}(2 \mathrm{~B})-\mathrm{C}(27 \mathrm{~B})$ & $108.0(3)$ \\
\hline $\mathrm{C}(11 \mathrm{~B})-\mathrm{Zr}(1 \mathrm{~B})-\mathrm{C}(5 \mathrm{~B})$ & $133.18(13)$ & $\mathrm{C}(28 \mathrm{~B})-\mathrm{Si}(2 \mathrm{~B})-\mathrm{C}(27 \mathrm{~B})$ & $105.6(3)$ \\
\hline $\mathrm{C}(3 \mathrm{~B})-\mathrm{Zr}(1 \mathrm{~B})-\mathrm{C}(5 \mathrm{~B})$ & $57.77(12)$ & $\mathrm{C}(12 \mathrm{~B})-\mathrm{Si}(2 \mathrm{~B})-\mathrm{C}(27 \mathrm{~B})$ & $115.5(2)$ \\
\hline $\mathrm{C}(2 \mathrm{~B})-\mathrm{Zr}(1 \mathrm{~B})-\mathrm{C}(5 \mathrm{~B})$ & $67.83(13)$ & $C(9 B)-S i(3 B)-C(31 B)$ & $108.31(18)$ \\
\hline $\mathrm{C}(12 \mathrm{~B})-\mathrm{Zr}(1 \mathrm{~B})-\mathrm{C}(5 \mathrm{~B})$ & $157.68(13)$ & $C(9 B)-S i(3 B)-C(30 B)$ & $113.58(18)$ \\
\hline $\mathrm{C}(10 \mathrm{~B})-\mathrm{Zr}(1 \mathrm{~B})-\mathrm{C}(5 \mathrm{~B})$ & $124.44(12)$ & $\mathrm{C}(31 \mathrm{~B})-\mathrm{Si}(3 \mathrm{~B})-\mathrm{C}(30 \mathrm{~B})$ & $108.4(2)$ \\
\hline
\end{tabular}




\begin{tabular}{|c|c|c|c|}
\hline C(9B)-Si(3B)-C(29B) & $112.9(2)$ & $\mathrm{C}(5 \mathrm{~B})-\mathrm{C}(6 \mathrm{~B})-\mathrm{Zr}(1 \mathrm{~B})$ & $73.69(19)$ \\
\hline $\mathrm{C}(31 \mathrm{~B})-\mathrm{Si}(3 \mathrm{~B})-\mathrm{C}(29 \mathrm{~B})$ & $105.8(2)$ & $\mathrm{C}(7 \mathrm{~B})-\mathrm{C}(6 \mathrm{~B})-\mathrm{Zr}(1 \mathrm{~B})$ & $136.0(3)$ \\
\hline $\mathrm{C}(30 \mathrm{~B})-\mathrm{Si}(3 \mathrm{~B})-\mathrm{C}(29 \mathrm{~B})$ & $107.5(2)$ & $\mathrm{C}(1 \mathrm{~B})-\mathrm{C}(6 \mathrm{~B})-\mathrm{Zr}(1 \mathrm{~B})$ & $65.5(2)$ \\
\hline C(7B)-Si(4B)-C(34B) & $116.66(17)$ & $\mathrm{C}(8 \mathrm{~B})-\mathrm{C}(7 \mathrm{~B})-\mathrm{C}(6 \mathrm{~B})$ & $104.7(3)$ \\
\hline C(7B)-Si(4B)-C(33B) & $107.42(18)$ & $\mathrm{C}(8 \mathrm{~B})-\mathrm{C}(7 \mathrm{~B})-\mathrm{Si}(4 \mathrm{~B})$ & $122.5(3)$ \\
\hline$C(34 B)-S i(4 B)-C(33 B)$ & $107.62(18)$ & $C(6 B)-C(7 B)-S i(4 B)$ & $131.2(3)$ \\
\hline C(7B)-Si(4B)-C(32B) & $111.60(19)$ & $\mathrm{C}(7 \mathrm{~B})-\mathrm{C}(8 \mathrm{~B})-\mathrm{C}(9 \mathrm{~B})$ & $113.3(3)$ \\
\hline $\mathrm{C}(34 \mathrm{~B})-\mathrm{Si}(4 \mathrm{~B})-\mathrm{C}(32 \mathrm{~B})$ & $105.28(19)$ & $\mathrm{C}(8 \mathrm{~B})-\mathrm{C}(9 \mathrm{~B})-\mathrm{C}(5 \mathrm{~B})$ & 104.6(3) \\
\hline $\mathrm{C}(33 \mathrm{~B})-\mathrm{Si}(4 \mathrm{~B})-\mathrm{C}(32 \mathrm{~B})$ & $107.93(18)$ & $\mathrm{C}(8 \mathrm{~B})-\mathrm{C}(9 \mathrm{~B})-\mathrm{Si}(3 \mathrm{~B})$ & $122.2(3)$ \\
\hline$C(19 B)-O(1 B)-C(20 B)$ & $112.9(4)$ & $\mathrm{C}(5 \mathrm{~B})-\mathrm{C}(9 \mathrm{~B})-\mathrm{Si}(3 \mathrm{~B})$ & $132.3(3)$ \\
\hline C(19B)-O(1B)-Zr(1B) & $124.7(3)$ & $\mathrm{C}(11 \mathrm{~B})-\mathrm{C}(10 \mathrm{~B})-\mathrm{C}(14 \mathrm{~B})$ & $104.0(4)$ \\
\hline C(20B)-O(1B)-Zr(1B) & $114.9(3)$ & $\mathrm{C}(11 \mathrm{~B})-\mathrm{C}(10 \mathrm{~B})-\mathrm{Si}(1 \mathrm{~B})$ & $126.4(3)$ \\
\hline $\mathrm{C}(22 \mathrm{~B})-\mathrm{O}(2 \mathrm{~B})-\mathrm{C}(21 \mathrm{~B})$ & $112.5(3)$ & $\mathrm{C}(14 \mathrm{~B})-\mathrm{C}(10 \mathrm{~B})-\mathrm{Si}(1 \mathrm{~B})$ & $124.9(3)$ \\
\hline $\mathrm{C}(22 \mathrm{~B})-\mathrm{O}(2 \mathrm{~B})-\mathrm{Zr}(1 \mathrm{~B})$ & $125.6(3)$ & $\mathrm{C}(11 \mathrm{~B})-\mathrm{C}(10 \mathrm{~B})-\mathrm{Zr}(1 \mathrm{~B})$ & $69.8(2)$ \\
\hline $\mathrm{C}(21 \mathrm{~B})-\mathrm{O}(2 \mathrm{~B})-\mathrm{Zr}(1 \mathrm{~B})$ & $120.0(3)$ & $\mathrm{C}(14 \mathrm{~B})-\mathrm{C}(10 \mathrm{~B})-\mathrm{Zr}(1 \mathrm{~B})$ & $79.7(2)$ \\
\hline$C(2 B)-C(1 B)-C(6 B)$ & $116.1(4)$ & $\mathrm{Si}(1 \mathrm{~B})-\mathrm{C}(10 \mathrm{~B})-\mathrm{Zr}(1 \mathrm{~B})$ & $133.94(17)$ \\
\hline C(2B)-C(1B)-Zr(1B) & $77.0(2)$ & $\mathrm{C}(10 \mathrm{~B})-\mathrm{C}(11 \mathrm{~B})-\mathrm{C}(12 \mathrm{~B})$ & $113.8(4)$ \\
\hline $\mathrm{C}(6 \mathrm{~B})-\mathrm{C}(1 \mathrm{~B})-\mathrm{Zr}(1 \mathrm{~B})$ & $80.5(3)$ & $\mathrm{C}(10 \mathrm{~B})-\mathrm{C}(11 \mathrm{~B})-\mathrm{Zr}(1 \mathrm{~B})$ & $76.8(2)$ \\
\hline $\mathrm{C}(3 \mathrm{~B})-\mathrm{C}(2 \mathrm{~B})-\mathrm{C}(1 \mathrm{~B})$ & $121.3(4)$ & $\mathrm{C}(12 \mathrm{~B})-\mathrm{C}(11 \mathrm{~B})-\mathrm{Zr}(1 \mathrm{~B})$ & $75.8(2)$ \\
\hline $\mathrm{C}(3 \mathrm{~B})-\mathrm{C}(2 \mathrm{~B})-\mathrm{Zr}(1 \mathrm{~B})$ & $72.5(3)$ & $C(11 B)-C(12 B)-C(13 B)$ & $103.5(3)$ \\
\hline $\mathrm{C}(1 \mathrm{~B})-\mathrm{C}(2 \mathrm{~B})-\mathrm{Zr}(1 \mathrm{~B})$ & $69.6(2)$ & $\mathrm{C}(11 \mathrm{~B})-\mathrm{C}(12 \mathrm{~B})-\mathrm{Si}(2 \mathrm{~B})$ & $121.8(3)$ \\
\hline $\mathrm{C}(2 \mathrm{~B})-\mathrm{C}(3 \mathrm{~B})-\mathrm{C}(4 \mathrm{~B})$ & $121.8(4)$ & $\mathrm{C}(13 \mathrm{~B})-\mathrm{C}(12 \mathrm{~B})-\mathrm{Si}(2 \mathrm{~B})$ & $127.2(3)$ \\
\hline $\mathrm{C}(2 \mathrm{~B})-\mathrm{C}(3 \mathrm{~B})-\mathrm{Zr}(1 \mathrm{~B})$ & $74.6(2)$ & $\mathrm{C}(11 \mathrm{~B})-\mathrm{C}(12 \mathrm{~B})-\mathrm{Zr}(1 \mathrm{~B})$ & $70.6(2)$ \\
\hline $\mathrm{C}(4 \mathrm{~B})-\mathrm{C}(3 \mathrm{~B})-\mathrm{Zr}(1 \mathrm{~B})$ & $68.7(2)$ & $\mathrm{C}(13 \mathrm{~B})-\mathrm{C}(12 \mathrm{~B})-\mathrm{Zr}(1 \mathrm{~B})$ & $79.5(2)$ \\
\hline$C(3 B)-C(4 B)-C(5 B)$ & $116.0(4)$ & $\mathrm{Si}(2 \mathrm{~B})-\mathrm{C}(12 \mathrm{~B})-\mathrm{Zr}(1 \mathrm{~B})$ & $137.63(17)$ \\
\hline C(3B)-C(4B)-Zr(1B) & $77.0(2)$ & $\mathrm{C}(18 \mathrm{~B})-\mathrm{C}(13 \mathrm{~B})-\mathrm{C}(14 \mathrm{~B})$ & $117.4(4)$ \\
\hline$C(5 B)-C(4 B)-Z r(1 B)$ & $82.0(2)$ & $\mathrm{C}(18 \mathrm{~B})-\mathrm{C}(13 \mathrm{~B})-\mathrm{C}(12 \mathrm{~B})$ & $132.7(4)$ \\
\hline $\mathrm{C}(6 \mathrm{~B})-\mathrm{C}(5 \mathrm{~B})-\mathrm{C}(9 \mathrm{~B})$ & $108.7(3)$ & $\mathrm{C}(14 \mathrm{~B})-\mathrm{C}(13 \mathrm{~B})-\mathrm{C}(12 \mathrm{~B})$ & 109.9(4) \\
\hline$C(6 B)-C(5 B)-C(4 B)$ & 119.1(4) & $\mathrm{C}(18 \mathrm{~B})-\mathrm{C}(13 \mathrm{~B})-\mathrm{Zr}(1 \mathrm{~B})$ & $124.3(2)$ \\
\hline C(9B)-C(5B)-C(4B) & $132.1(4)$ & $\mathrm{C}(14 \mathrm{~B})-\mathrm{C}(13 \mathrm{~B})-\mathrm{Zr}(1 \mathrm{~B})$ & $75.41(19)$ \\
\hline $\mathrm{C}(6 \mathrm{~B})-\mathrm{C}(5 \mathrm{~B})-\mathrm{Zr}(1 \mathrm{~B})$ & $74.04(19)$ & $\mathrm{C}(12 \mathrm{~B})-\mathrm{C}(13 \mathrm{~B})-\mathrm{Zr}(1 \mathrm{~B})$ & $68.02(19)$ \\
\hline C(9B)-C(5B)-Zr(1B) & $135.3(3)$ & $\mathrm{C}(15 \mathrm{~B})-\mathrm{C}(14 \mathrm{~B})-\mathrm{C}(13 \mathrm{~B})$ & $119.5(4)$ \\
\hline $\mathrm{C}(4 \mathrm{~B})-\mathrm{C}(5 \mathrm{~B})-\mathrm{Zr}(1 \mathrm{~B})$ & $64.2(2)$ & $C(15 B)-C(14 B)-C(10 B)$ & $131.9(4)$ \\
\hline $\mathrm{C}(5 \mathrm{~B})-\mathrm{C}(6 \mathrm{~B})-\mathrm{C}(7 \mathrm{~B})$ & 108.6(3) & $\mathrm{C}(13 \mathrm{~B})-\mathrm{C}(14 \mathrm{~B})-\mathrm{C}(10 \mathrm{~B})$ & $108.5(4)$ \\
\hline $\mathrm{C}(5 \mathrm{~B})-\mathrm{C}(6 \mathrm{~B})-\mathrm{C}(1 \mathrm{~B})$ & $119.8(3)$ & $\mathrm{C}(15 \mathrm{~B})-\mathrm{C}(14 \mathrm{~B})-\mathrm{Zr}(1 \mathrm{~B})$ & $127.4(2)$ \\
\hline $\mathrm{C}(7 \mathrm{~B})-\mathrm{C}(6 \mathrm{~B})-\mathrm{C}(1 \mathrm{~B})$ & $131.6(4)$ & $\mathrm{C}(13 \mathrm{~B})-\mathrm{C}(14 \mathrm{~B})-\mathrm{Zr}(1 \mathrm{~B})$ & $73.3(2)$ \\
\hline
\end{tabular}




$\begin{array}{lcl}\mathrm{C}(10 \mathrm{~B})-\mathrm{C}(14 \mathrm{~B})-\mathrm{Zr}(1 \mathrm{~B}) & 67.93(19) & \mathrm{O}(2 \mathrm{~B})-\mathrm{C}(21 \mathrm{~B})-\mathrm{C}(20 \mathrm{~B}) \\ \mathrm{C}(16 \mathrm{~B})-\mathrm{C}(15 \mathrm{~B})-\mathrm{C}(14 \mathrm{~B}) & 121.1(5) & \\ \mathrm{C}(15 \mathrm{~B})-\mathrm{C}(16 \mathrm{~B})-\mathrm{C}(17 \mathrm{~B}) & 120.6(5) & \\ \mathrm{C}(18 \mathrm{~B})-\mathrm{C}(17 \mathrm{~B})-\mathrm{C}(16 \mathrm{~B}) & 120.4(5) & \text { Symmetry transformations used to generate } \\ \mathrm{C}(17 \mathrm{~B})-\mathrm{C}(18 \mathrm{~B})-\mathrm{C}(13 \mathrm{~B}) & 121.0(4) & \text { equivalent atoms: } \\ \mathrm{O}(1 \mathrm{~B})-\mathrm{C}(20 \mathrm{~B})-\mathrm{C}(21 \mathrm{~B}) & 107.4(4) & \end{array}$




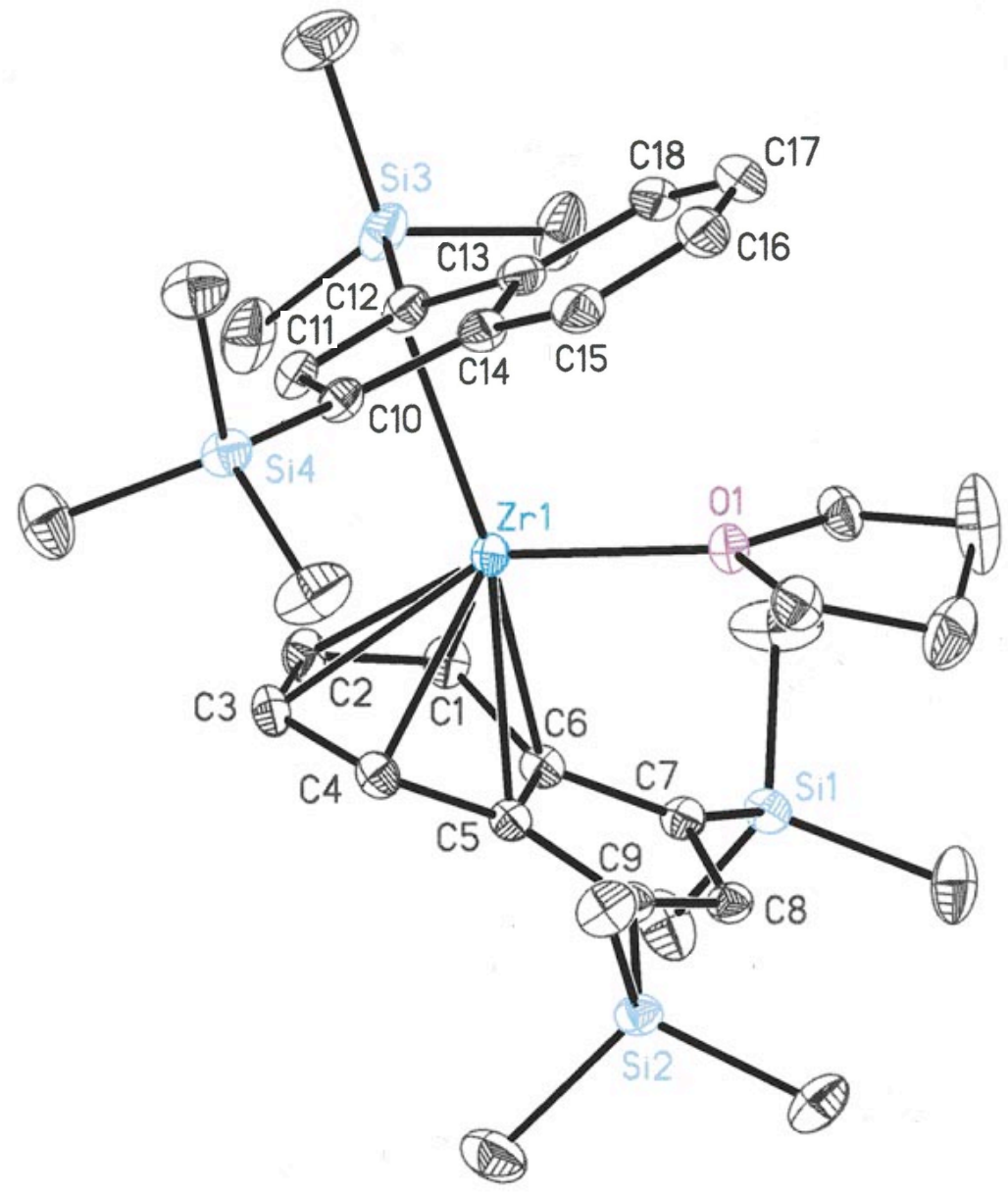

Figure S7. Partially labeled view of the molecular structure of $\mathbf{1 - T H F}$ with $30 \%$ probability ellipsoids and hydrogen atoms omitted for clarity. 


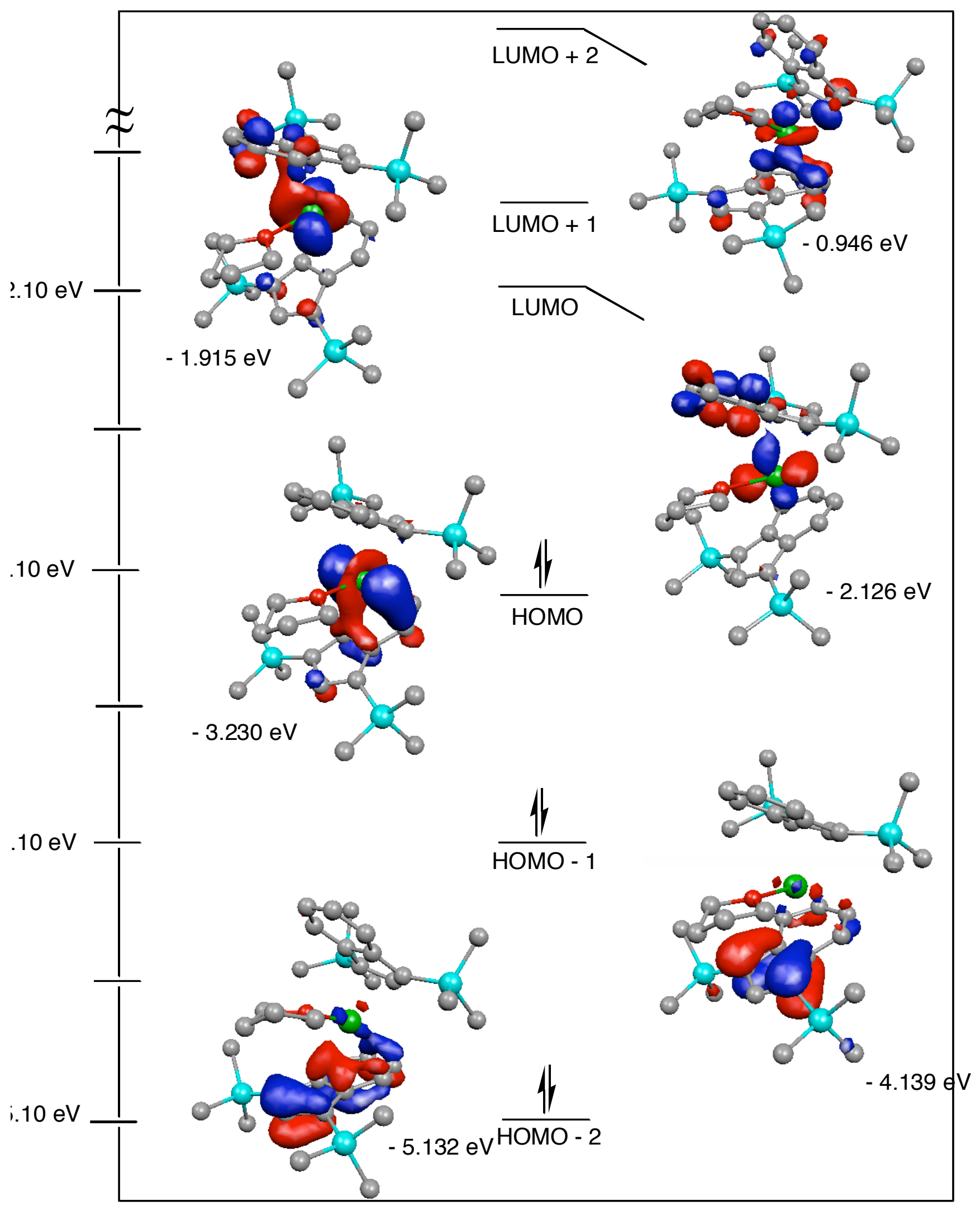

Figure S8. Frontier molecular orbital diagram of 1-THF as calculated using the ADF program suite (TZ2P, ZORA). 


\section{References.}

${ }^{1}$ Pangborn, A. B.; Giardello, M. A.; Grubbs, R. H.; Rosen, R. K.; Timmers, F. J. Organometallics 1996, 15, 1518.

${ }^{2}$ Bradley, C. A.; Keresztes, I.; Lobkovsky, E.; Young, V. G.; Chirik, P. J. J. Am. Chem. Soc. 2004, 126, 16937.

${ }^{3}$ Bradley, C. A.; Lobkovsky, E.; Chirik, P. J. J. Am. Chem. Soc. 2003, 125, 8110.

${ }^{4}$ McElvain, S. M.; Stammer, C. H. J. Am. Chem. Soc. 1951, 73, 915.

${ }^{5}$ Cohen, H. L.; Wright, G. F. J. Org. Chem. 1953, 18, 432.

${ }^{6}$ Gómez, J. C. C.; López, F. J. S. MestRe-C, Version 3.71.9.0; Universidade de Santiago de Compostela, 2004. http://www.mestrec.com.

${ }^{7}$ a) Perrin, C. L.; Dwyer, T. J. Chem. Rev. 1990, 90, 935. b) Derose, E. F.; Castillo, J.; Saulys, D.; Morrison, J. J. Mag. Res. 1991, 93, 347. c) Zolnai, Z.; Juranic, N.; VikicTopic, D.; Macura, S. J. Chem. Inf. Comput. Sci. 2000, 40, 611. 